\title{
RESEARCH ARTICLE \\ Characterising scoring rules by their solution in iteratively undominated strategies
}

\section{Christian Basteck ${ }^{1}$}

Received: 4 July 2019 / Accepted: 13 February 2021 / Published online: 9 April 2021

(c) The Author(s) 2021

\begin{abstract}
We characterize voting procedures according to the social choice correspondence they implement when voters cast ballots strategically, applying iteratively undominated strategies. In elections with three candidates, the Borda Rule is the unique positional scoring rule that satisfies unanimity (U) (i.e., elects a candidate whenever it is unanimously preferred) and is majoritarian after eliminating a worst candidate (MEW)(i.e., if there is a unanimously disliked candidate, the majority-preferred among the other two is elected). In a larger class of rules, Approval Voting is characterized by a single axiom that implies both $\mathrm{U}$ and MEW but is weaker than Condorcet-consistency $(\mathrm{CON})$ - it is the only direct mechanism scoring rule that is majoritarian after eliminating a Pareto-dominated candidate (MEPD)(i.e., if there is a Pareto-dominated candidate, the majority-preferred among the other two is elected); among all finite scoring rules that satisfy MEPD, Approval Voting is the most decisive. However, it fails a desirable monotonicity property: a candidate that is elected for some preference profile, may lose the election once she gains further in popularity. In contrast, the Borda Rule is the unique direct mechanism scoring rule that satisfies U, MEW and monotonicity (MON). There exists no direct mechanism scoring rule that satisfies both MEPD and MON and no finite scoring rule satisfying CON.
\end{abstract}

Keywords Sophisticated voting - Iterated weak dominance · Majoritarianism · Scoring rules $\cdot$ Borda rule $\cdot$ Approval voting

JEL Classification C72, D71, D72

Christian Basteck

christian.basteck@wzb.eu

1 WZB Berlin Social Science Center, Berlin, Germany 


\section{Introduction}

Majority rule is often considered the hallmark of democratic decision making and has been axiomatised by May (1952) for the two-candidate case. It is however unclear how it should be adapted to situations involving more than two candidates. First, with at least three candidates, it is often unclear who should be considered 'majority-preferred' even when voters' true preferences are known, say because a Condorcet-winner may fail to exist. Second, voting procedures that take a majority of voters' preferences into account—and are hence non-dictatorial—are necessarily manipulable. For voting procedures that always yield a unique winner, this was first shown by Gibbard (1973) and Satterthwaite (1975) and allowing for ties does not resolve the problem, see e.g. Gärdenfors (1976), Barberà et al. (2001) or Ching and Zhou (2002). Strategic voting may then drive a wedge between reported and true preferences of voters and sever the link between election outcomes and voters' preferences.

In this paper, we identify voting procedures that (i) ensure the election of a 'majoritypreferred' among three candidates for preferences profiles where such a candidate can be clearly identified, despite the fact that (ii) voters may cast their ballots strategically, potentially misrepresenting their preferences. Hence, rather than analysing either the preference aggregation properties of a voting rule (implicitly assuming sincere voting) or the extent of a voting rule's manipulability ${ }^{1}$ (without checking for the effect that manipulations have on the eventual election outcomes) in isolation, we analyse both issues jointly to see how severe the inescapable problem of strategic misrepresentation of preferences is with respect to giving rise to undesirable election outcomes.

With respect to (i), we will consider various axioms. For example, at the very least, if all voters agree that one of three candidates is the least preferred, the majoritypreferred among the other two should be elected. More ambitiously, we may ask that whenever there are two Pareto-efficient alternatives, the majority-preferred among the two should be chosen. Yet more demanding, we may stipulate that a Condorcet-winner is chosen whenever it exists. Alongside these requirements we will consider familiar unanimity and monotonicity axioms. ${ }^{2}$

With respect to (ii), we will assume that voters only use iteratively undominated strategies. That is, we rely on iterative elimination of (weakly) dominated strategies as our solution concept. The concept has a long tradition in the theory of voting where it was introduced by Farquharson (1969) under the name of sophisticated voting. It is particularly well suited to model strategic behaviour in elections where the number of voters is large relative to the number of available alternatives, as under these conditions voters typically find themselves in a position where they are not pivotal. As a result any strategy is a best response and the alternative solution concept of rationalizability has no bite. In particular, under many intuitive voting procedures, if all voters vote

\footnotetext{
1 Analysis in this vein includes checking for the number of preference profiles that allow for profitable manipulations (Kelly 1993; Lepelley and Merlin 2001), studying the size of potential gains from manipulations (Campbell and Kelly 2009) or identifying non-manipulable (sub)domains (Barbie et al. 2006; Sanver 2009).

2 May (1952) also invokes monotonicity, referring to it as positive responsiveness, in his characterisation of the majority rule.
} 
'in favour' of some arbitrary alternative, this alternative is elected and no individual is pivotal. But then any alternative may be chosen in (some) Nash-equilibrium.

As the set of voting procedures under consideration, we will consider scoring rules, i.e., voting procedures where each voter awards scores to candidates and the candidate with the highest aggregate score wins the election. In the class of all neutral and anonymous voting procedures, scoring rules are the only procedures that satisfy reinforcement ${ }^{3}$ and overwhelming majority, ${ }^{4}$ see (Myerson 1995). They include prominent rules such as 'classical' positional scoring rules, for example the (Anti-)Plurality Rule or the Borda Rule, other rules with a similarly small strategy space such as Approval Voting and rules with an arbitrarily large strategy space such as Evaluative Voting and Cumulative Voting.

Within this class of voting procedures, we are able to derive three main characterisation results. First, in the subset of positional scoring rules, the Borda Rule is the unique voting procedure that satisfies unanimity (U) (i.e., uniquely selects an alternative whenever it is unanimously preferred) and is majoritarian after eliminating a worst alternative (MEW) (i.e., if there is a unanimously disliked alternative, the majority-preferred among the other two alternatives is uniquely selected).

Second, in the class of direct mechanism scoring rules (including, e.g., all positional scoring rules and Approval Voting), the Borda Rule is the unique voting procedure implementing a social choice correspondence that satisfies U, MEW and monotonicity (MON)(i.e., an alternative that is uniquely selected for some preference profile should still be uniquely selected when every voter ranks this alternative weakly higher).

Third, in the class of all finite scoring rules (i.e., scoring rules with arbitrarily but finitely many admissible ballots) extensions of Approval Voting are the only voting procedures that are majoritarian after eliminating a Pareto-dominated alternative (MEPD) (i.e., if there is a Pareto-dominated alternative, the majority-preferred alternative among the other two is uniquely selected) - and Approval Voting is characterized not only as the simplest such rule but also as the most decisive.

As a novel voting paradox, we find that Approval Voting violates MON and MON* (i.e., an alternative that is included in the solution for some preference profile should still be included for a preference profile where every voter ranks this alternative weakly higher). More generally, no finite scoring rule will satisfy both MON* and MEPD, nor does there exist a finite scoring rule that is Condorcet-consistent. The fact that the familiar notion of Condorcet-consistency (CON) is unachievable in conjunction with strategic voting underlines the importance of the criteria MEW and MEPD which both weaken CON just enough so as to allow for possibility results. Finally, as an intermediate product for our characterization, we delineate those preference profiles for which Borda Rule voting games are dominance-solvable.

Three papers most closely related to this work are (Dhillon and Lockwood 2004), (Buenrostro et al. 2013) and (Courtin and Núñez 2017) who all identify conditions for preferences profiles under which particular scoring rules yield a unique solution

\footnotetext{
3 If ballots are evaluated for separate districts and the same alternative is chosen in each, then in a joint district, i.e., for the joined ballot profile, this alternative is chosen as well.

4 If some group of voters, or rather the ballots that they cast, are replicated sufficiently often, an election outcome at the resulting ballot profile has to be a possible outcome (possibly tied) at the profile where only ballots of the replicated group are considered.
} 
in iteratively undominated strategies. Dhillon and Lockwood (2004) consider Plurality Rule voting games with an arbitrary number of alternatives and provide sufficient as well as necessary conditions. They find that if the preference profile is such that the game is dominance-solvable, then there exists a Condorcet-winner. Moreover, if their sufficient condition for dominance-solvability is satisfied, the unique outcome is the Condorcet-winner. ${ }^{5}$ Buenrostro et al. (2013) consider "general scoring rules" and provide sufficient conditions. They show that if their sufficient conditions for dominance-solvability are satisfied, the unique outcome is a Condorcet-winner. Courtin and Núñez (2017) consider Approval Voting, provide sufficient as well as necessary conditions for dominance-solvability, and show that under their sufficient conditions, the unique outcome is a Condorcet-winner.

In contrast to these previous works, we also consider weaker notions of majoritarianism besides Condorcet-consistency and identify scoring rules that uniquely elect such 'majority preferred candidates' whenever they exist - rather than narrowing down the set of preference profiles in advance by additional sufficient criteria.

Myerson (2002) considers scoring rules in Poisson voting games. He finds that, in equilibrium, Approval Voting ensures the election of a majority-preferred among two Pareto-efficient candidates as the size of the electorate goes to infinity. Using iterative elimination of dominated strategies as solution concept, we confirm the finding for finite electorates. More importantly, we show that Approval Voting can be characterized by this advantageous preference aggregation property - it is the unique direct mechanism scoring rule and the most decisive among all finite scoring rules that satisfies this property.

The paper is organised as follows. Section 2 defines voting games and their solution by iterative elimination of dominated strategies. Section 3 defines normative criteria for social choice correspondences. Section 4 characterizes scoring rules with respect to the social choice correspondences that they implement. Section 5 concludes.

\section{Technicalities}

\subsection{Candidates and voters}

Throughout the paper, we consider a set of three candidates (or alternatives) $A=$ $\{a, b, c\}$ and a finite set of voters $I$ with generic element $i$. Each voter's preferences are assumed to be given by a strict linear order $\succ_{i}$ on $A$. In consequence, there are six distinct sets of voters, characterized by their preferences, that we denote $I_{x y z}=\{i \in$ $\left.I \mid x, y, z \in A, x \succ_{i} y \succ_{i} z\right\}$. A preference profile is denoted as $\succ_{I}=\left(\succ_{i}\right)_{i \in I}$.

\subsection{Scoring rules}

Scoring rules allow each voter $i$ to cast a ballot $v_{i}=\left(v_{i}^{a}, v_{i}^{b}, v_{i}^{c}\right)$ from the same set of admissible ballots $V_{i}=V \subset \mathbb{R}^{3}$. We assume that ballots are neutral with respect

\footnotetext{
5 They conjecture that whenever the game is dominance-solvable, the unique outcome is the Condorcetwinner.
} 
to a relabelling of candidates; formally, for any admissible ballot $v_{i}=(k, l, m) \in V$, each permutation of $v_{i}$ is also an admissible ballot. A ballot is called an abstention if it takes the form $v_{i}=(k, k, k)$.

Using Cartesian products, we define $V^{0}=\prod_{i \in I} V_{i}$ and $V_{-i}^{0}=\prod_{j \neq i} V_{j}$. We refer to $v \in V^{0}$ as a ballot profile and denote the associated score of some candidate $x$ as $v^{x}=\sum_{i \in I} v_{i}^{x}$. For an opposing ballot profile $v_{-i} \in V_{-i}^{0}$ we define $v_{-i}^{x}=\sum_{j \neq i} v_{j}^{x}$.

A candidate wins the election if her score is higher than any other candidate's score. To deal with ties, we rely on the report of a tiebreaker, labelled $t$, who has to choose a strict linear order $\triangleright$ on $A$, where $\triangleright$ denotes the set of such orders. Then, for given $v$ and $\triangleright$, candidate $x$ wins the election whenever she has a weakly higher score than all other candidates and, in case of a tie, is ranked first according to $\triangleright$. Formally, $x$ wins if and only if

$$
\forall y \neq x:(1) v^{x} \geq v^{y} \text {; and (2) } v^{x}=v^{y} \Longrightarrow x \triangleright y,
$$

so that there is a unique winner for any ballot profile $v$ and tiebreaker report $\triangleright$. Since voters' identities affect neither candidates' scores nor the way in which ties are broken, the procedure is (voter-)anonymous.

Importantly, the set of possible reports by the indifferent tiebreaker is not narrowed down by iterative elimination of weakly dominated strategies. ${ }^{6}$ Moreover, a voter may rule out a strategy in the process of iterative elimination, only if it leads to weakly worse outcomes under every possible tiebreaker report $\triangleright \in D$. Hence, our modelling choice makes voters more cautious in ruling out particular strategies than if we assumed a fixed deterministic tiebreaker, say $a \triangleright b \triangleright c$, a property that may be seen as befitting the cautious nature in which the iterative elimination of weakly dominated strategies proceeds in general. In addition, by fixing a particular tiebreaker we would forego neutrality as candidates would no longer be treated symmetrically.

If instead we would refrain from breaking ties in a deterministic manner, outcomes would either be set-valued or take the form of a lottery over alternatives. For the former, one would then have to amend voters preferences so as to include preferences over sets of (tied) candidates. Here the following Gärdenfors extension (Sanver and Zwicker 2012) is among the best known: ${ }^{7}$ given a strict linear order $\succ$ on $A$, define for all nonempty $A^{\prime}, A^{\prime \prime} \subseteq A$

$$
\begin{aligned}
& A^{\prime} \succ^{G} A^{\prime \prime} \quad: \Longleftrightarrow A^{\prime} \neq A^{\prime \prime} \text { and } \forall x \in A^{\prime} \backslash A^{\prime \prime}, y \in A^{\prime \prime}: x \succ y \\
& \text { and } \forall x \in A^{\prime}, y \in A^{\prime \prime} \backslash A^{\prime}: x \succ y \text {. }
\end{aligned}
$$

\footnotetext{
6 If one objects to the introduction of an additional player, one could alternatively break ties by a multiplayer version of "matching pennies": ask each voter to report a number $t_{i} \in\{0,1, \ldots, 5\}$, set $t=\sum t_{i} \bmod 6$ and let each possible outcome $t=\{0,1, \ldots, 5\}$ correspond to one of the 6 possible linear orders $\triangleright \in D$. For our purposes, the two approaches are equivalent, as a voters' set of possible reports $t_{i}$ would likewise not be reduced by (iterative) elimination of weakly dominated strategies. However anonymity would then be satisfied for all players (rather than 'only' for voters).

7 It formalizes the "sure-thing principle” of Gärdenfors (1976); see also Gärdenfors (1979).
} 
In our setting where preferences over candidates are strict, this approach is equivalent to the one persued by Brams and Fishburn (1978). ${ }^{8}$ For another equivalent extension see Ching and Zhou (2002, Lemma 1). ${ }^{9}$ Importantly, if we would define a strategy to be weakly dominated whenever it yields the same or a worse (according to $\succ^{G}$ ) set of candidates against any opposing voter strategy profile, this would be equivalent to our approach of invoking an indifferent tiebreaker, since one can show that one set of (tied) candidates is preferred to another according to $\succ^{G}$ if and only if it yields the same or a better outcome for any possible tiebreaker $\triangleright$.

Finally, if instead we allowed outcomes to be lotteries over alternatives by specifying a random tie-breaking procedure and associated voters with von Neumann Morgenstern utility functions to evaluate such lotteries, not only would this increase the amount of preference information required in the analysis, but also create a technical difficulty: "transference of decisionmaker indifference" (Marx and Swinkels 1997) would not be guaranteed. ${ }^{10}$ As a result, the solution in iteratively undominated strategies could depend on the order in which dominated strategies are eliminated, see Example 4 in the Appendix. By contrast, our approach ensures order independence, see Sect. 2.5.

Among the most prominent scoring rules are so called positional scoring rules, ${ }^{11}$ i.e., rules that let voters rank candidates and assign scores based on that ranking the highest score to the top-ranked, the lowest score to the last-ranked candidate. Formally, the set of voters' admissible ballots $V$ can be taken to be all permutation of $(1, s, 0)$, where $s \in[0,1]$ is a fixed parameter that characterizes the rule. The most notable positional scoring rules are the Plurality Rule, corresponding to $s=0$, the Antiplurality Rule $(s=1)$ and the Borda Rule $\left(s=\frac{1}{2}\right)$.

Note that positional scoring rules are direct mechanisms, in that a voter's preferences over candidates can be mapped naturally to a particular strategy. To see which normative properties can be satisfied without having to resort to more complex mechanisms, we will analyse these 'classical' scoring rules along with other scoring rules that can be interpreted as direct mechanism, i.e., where the size of voters' strategy space is bounded by the number of voters' types. Formally, a scoring rule as described above is a direct mechanism scoring rule if, after the removal of abstentions, ${ }^{12}$ we have $|V| \leq 6$.

Beyond positional scoring rules, direct mechanism scoring rules may allow voters to either vote for one candidate or split their vote between two - we refer to such rules as vote-splitting scoring rules. Formally, $V$ then consists of all permutations of $(s, s, 0)$ and $(1-s, 0,0), s \in[0,1]$. If $s=\frac{1}{3}$, voters have a fixed budget of points that they may award to a single candidate or split in two. Setting $s=\frac{1}{2}$ corresponds to

\footnotetext{
${ }^{8}$ For a strict linear order on $A$ their extension $R$ satisfies $A^{\prime} R A^{\prime \prime} \Longleftrightarrow A^{\prime}=A^{\prime \prime}$ or $A^{\prime} \succ^{G} A^{\prime \prime}$.

9 They consider $A^{\prime}$ preferred over $A^{\prime \prime}$ iff it yields weakly higher expected utility for every subjective probability measure used to randomly break ties and for every von Neumann - Morgenstern utility function consistent with preferences. For strict preferences, this holds iff $A^{\prime}=A^{\prime \prime}$ or $A^{\prime} \succ^{G} A^{\prime \prime}$.

10 Voter $i$ may be indifferent between $b$ and a tie of $a$ and $c$, while others' preferences are strict.

11 In fact, they are frequently referred to simply as scoring rules (Moulin 1991; Lepelley and Merlin 2001; Baharad and Nitzan 2005, 2007), as scoring methods (Young 1995), or as 'classical' scoring rules (Dietrich 2014).

12 Since abstentions are dominated strategies, removing them will not affect our analysis.
} 
Approval Voting. Note that $s=1$ is equivalent to the Antiplurality Rule, while $s=0$ corresponds to the Plurality Rule. Hence, both Approval Voting and the Borda Rule can be thought of as 'half-way' between the Plurality and Antiplurality Rule. Our first result will show that positional and vote-splitting scoring rules are essentially the only direct mechanism scoring rules.

Finally, we will further broaden the scope of our analysis and consider all finite scoring rules, i.e., rules for which $|V| \in \mathbb{N}$, to see what can and cannot be achieved by allowing for more complex rules. In a slight abuse of notation, we will at times identify a scoring rule and the set of admissible ballots and denote both by $V$.

\subsection{Voting games}

Together, the set of candidates, voters' preferences, a scoring rule and a tiebreakerassumed to be indifferent between candidates-give rise to a complete information voting game $\Gamma\left(\succ_{I}, V^{0}\right)$ with a set of players $I \cup\{t\}$. In each game $\Gamma\left(\succ_{I}, V^{0}\right)$, a strategy profile $(v, \triangleright) \in V^{0} \times \triangleright$ determines a unique outcome $g(v, \triangleright) \in A$.

We will also consider restricted games $\Gamma\left(\succ_{I}, V^{\prime}\right)$, where each voter's strategies are restricted to some set $V_{i}^{\prime} \subseteq V$ and the space of ballot profiles is denoted $V^{\prime}=\prod_{i \in I} V_{i}^{\prime}$. Accordingly, the space of opponents' ballot profiles is denoted $V_{-i}^{\prime}=\prod_{j \neq i} V_{j}^{\prime}$. Where all voters $i \in I_{x y z}$ have the same (restricted) strategy set, we denote it $V_{x y z}^{\prime}=V_{i}^{\prime}$.

\subsection{Iteratively undominated strategies}

In particular, we will focus on restricted games where weakly dominated strategies have been removed.

Definition 1 A strategy $v_{i} \in V_{i}^{\prime}$ is weakly dominated in $\Gamma\left(\succ_{I}, V^{\prime}\right)$ if there exists $\tilde{v}_{i} \in V_{i}^{\prime}$ such that for all $v_{-i} \in V_{-i}^{\prime}, \triangleright \in \triangleright$

$$
g\left(\tilde{v}_{i}, v_{-i}, \triangleright\right) \succ_{i} g\left(v_{i}, v_{-i}, \triangleright\right) \text { or } g\left(\tilde{v}_{i}, v_{-i}, \triangleright\right)=g\left(v_{i}, v_{-i}, \triangleright\right)
$$

with $g\left(\tilde{v}_{i}, v_{-i}, \triangleright\right) \succ_{i} g\left(v_{i}, v_{-i}, \triangleright\right)$ for at least one $v_{-i} \in V_{-i}^{\prime}$ and $\triangleright \in \triangleright$.

Strategies $\triangleright \in D$ are never dominated, as the tiebreaker is assumed to be indifferent between all outcomes $g(v, \triangleright) \in A$. Hence, in iteratively removing dominated strategies, we can focus on voters $i \in I$. First, define the set of undominated strategies as $V_{i}^{1}=V \backslash\left\{v_{i} \in V \mid v_{i}\right.$ is weakly dominated in $\left.\Gamma\left(\succ_{I}, V^{0}\right)\right\}$.

Next, move to the iterative elimination of dominated strategies and define

$$
V_{i}^{m+1}=V_{i}^{m} \backslash\left\{v_{i} \in V_{i}^{m} \mid v_{i} \text { is weakly dominated in } \Gamma\left(\succ_{I}, V^{m}\right)\right\} \text {, for } m \in \mathbb{N} \text {. }
$$

Since $\succ_{i}$ is acyclic, $V_{i}^{m+1}$ contains at least one strategy, undominated in $\Gamma\left(\succ_{I}, V^{m}\right)$. Also, as $V$ is finite, there exists some $\bar{m}$, such that no further restrictions are possible; $V^{m}=V^{\bar{m}}$, for all $m \geq \bar{m}$. This leads us to the following solution of a voting game. 
Definition 2 For a voting game $\Gamma\left(\succ_{I}, V^{0}\right)$ we define its solution in iteratively undominated strategies as the set of possible outcomes after iteratively eliminating all weakly dominated strategies, and denote it as

$$
S\left(\succ_{I}, V\right)=\left\{x \in A \mid \exists v \in V^{\bar{m}}: \forall y \in A: v^{x} \geq v^{y}\right\} .
$$

We say that $V$ implements the social choice correspondence $S(\cdot, V)$ that maps preference profiles onto subsets of $A$.

\subsection{Order independence and elimination of redundant strategies}

In specifying the solution concept above, we followed Moulin (1979) in that we eliminated all weakly dominated strategies when moving from $V^{m}$ to $V^{m+1} .^{13}$ This raises the question, whether a different order of elimination, where only some dominated strategies are removed at each step, might yield a different solution.

Fortunately, Marx and Swinkels (1997) assure us that this is not the case. More precisely, their Theorem 1 ensures that once we reach a restricted game $\Gamma\left(\succ_{I}, V^{\prime}\right)$ such that no further strategy can be eliminated based on weak dominance, $\Gamma\left(\succ_{I}, V^{\prime}\right)$ will be equivalent to $\Gamma\left(\succ_{I}, V^{\bar{m}}\right)$ up to the elimination of redundant strategies ${ }^{14}$ and the renaming of strategies. In particular, the set of possible outcomes of both games will be the same.

This is because, in our voting games, the elimination of dominated strategies satisfies what Marx and Swinkels (1997) call "transference of decisionmaker indifference": whenever a voter $i$, for a given opposing strategy profile, is indifferent between outcomes $g\left(v_{i}, v_{-i}, \triangleright\right)$ and $g\left(\tilde{v}_{i}, v_{-i}, \triangleright\right)$, then so is every other player. This is of course satisfied, as $i$ will only be indifferent if both outcomes coincide. ${ }^{15}$

Moreover, whether in the process of iterative elimination, we at some point choose to eliminate one of multiple redundant undominated strategies, will be of no effect; the game $\Gamma\left(\succ_{I}, V^{\prime}\right)$ that we reach eventually will be equivalent to $\Gamma\left(\succ_{I}, V^{\bar{m}}\right)$ up to the elimination of redundant strategies and the renaming of strategies.

To see this, suppose that in the game $\Gamma\left(\succ_{I}, V^{m}\right)$ there are two redundant but undominated strategies $v_{i}, \tilde{v}_{i} \in V_{i}^{m}$, of which we choose to eliminate only $\tilde{v}_{i}$ when moving to the next restricted game. If $\tilde{v}_{i}$ could at some step be instrumental in eliminating another strategy $v_{j}$ based on weak dominance, the remaining strategy $v_{i}$ will suffice to eliminate $v_{j}$. If $v_{i}$ was eliminated based on weak dominance before it becomes instrumental in eliminating $v_{j}, \tilde{v}_{i}$ would have been eliminated as well.

\footnotetext{
13 Farquharson (1969) invokes the same solution, referring to it as "sophisticated voting."

14 A strategy $\tilde{v}_{i}$ is redundant to $v_{i}$ in $\Gamma\left(\succ_{I}, W\right), W \subseteq V^{0}$, iff, for any opposing strategy profile $\left(v_{-i}, \triangleright\right) \in$ $W_{-i} \times \triangleright$, they lead to the same outcome $g\left(\tilde{v}_{i}, v_{-i}, \triangleright\right)=g\left(v_{i}, v_{-i}, \triangleright\right) \in A$ (see Definition 5 in Marx and Swinkels 1997).

15 Indifference of the tiebreaker does not transfer to indifference of other voters. However, this is unproblematic, as transference is only required to hold for players whose strategies are eliminated (see Definition 2 in Marx and Swinkels 1997).
} 


\section{Axioms}

We want to compare and characterize scoring rules according to the social choice correspondences that they implement. In particular, we ask for which preference profiles the induced voting games have a unique solution - and which outcomes are selected in that case. A minimal and prominent requirement is unanimity.

Definition 3 A scoring rule $V$ is said to satisfy Unanimity (U), if for any preference profile $\succ_{I}$ such that $I=I_{x y z} \cup I_{x z y}$, we have $S\left(\succ_{I}, V\right)=\{x\}$.

Where there is no universal agreement, we have to weigh some voters' preferences against others', to choose between alternatives. In the case of two alternatives, fairness and efficiency force us to accept simple majority as guiding principle, ${ }^{16}$ but adapting this principle to accommodate more alternatives is not immediate.

However, if one of three alternatives is unanimously agreed to be the worst, one may argue that there are only two relevant alternatives, so that a decision should again be made by simple majority. We formalize this idea as follows.

Definition 4 Consider any preference profile $\succ_{I}$ where $z$ is unanimously considered to be the worst candidate, i.e., $I=I_{x y z} \cup I_{y x z}$. A scoring rule $V$ is said to be Majoritarian after Eliminating a Worst Alternative (MEW), if $\left|I_{x y z}\right|>\left|I_{y x z}\right|$ implies $S\left(\succ_{I}, V\right)=$ $\{x\}$.

Where the choice between two competing candidates, or parties offering alternative policies, would follow majority rule, our axiom requires that the introduction of a third, universally disliked alternative should not affect the outcome of the vote. Hence, it limits parties' incentives to strategically distort the set of alternatives.

A similar situation arises when one of three alternatives is unanimously agreed to be worse than some other alternative. For example, one of two candidates with identical policy positions may be more corrupt than the other, as considered by Myerson (2002). Again, one might hope that the Pareto-dominated candidate is disregarded and the decision between the remaining two made by simple majority.

Definition 5 Consider any preference profile $\succ_{I}$ such $z$ is Pareto-dominated by $x$, i.e., $I=I_{x y z} \cup I_{x z y} \cup I_{y x z}$. A scoring rule $V$ is said to be Majoritarian after Eliminating $a$ Pareto-Dominated Alternative (MEPD), if $\left|I_{x y z}\right|+\left|I_{x z y}\right|>\left|I_{y x z}\right|$ implies $S\left(\succ_{I}, V\right)=$ $\{x\}$, while $\left|I_{x y z}\right|+\left|I_{x z y}\right|<\left|I_{y x z}\right|$ implies $S\left(\succ_{I}, V\right)=\{y\}$.

The formal definition reveals what might be a controversial property of MEPD: some alternative $y$ might be chosen by the social choice correspondence $S(\cdot, V)$ based on its majority support over another alternative $x$, even though it may only be $x$ that, according to MEPD, forces us to eliminate $z$, based on Pareto-dominance.

In defence of MEPD, observe that it unifies both preceding axioms, i.e., implies both MEW and $\mathbf{U}$. Moreover, it is implied by another, well known requirement, formulated by Condorcet, according to which an alternative should be chosen whenever it is majority-preferred over any other alternative. ${ }^{17}$

\footnotetext{
16 May (1952) provides an axiomatisation of the Majority Rule. His symmetry axioms can be seen as an embodiment of fairness, while positive responsiveness may be seen as a requirement of efficiency.

17 Of course, such an alternative may fail to exist if pairwise comparisons yield a cycle, see Condorcet (1785), p. lxi.
} 
Fig. 1 Logical relations between intra-profile axioms

Definition 6 A scoring rule $V$ is Condorcet-consistent (CON), if $S\left(\succ_{I}, V\right)=\{x\}$ whenever $x$ is a Condorcet-winner for preference profile $\succ_{I}$, i.e., whenever

$$
\begin{gathered}
\left|I_{x y z}\right|+\left|I_{x z y}\right|+\left|I_{y x z}\right|>\left|I_{y z x}\right|+\left|I_{z x y}\right|+\left|I_{z y x}\right| \text { and } \\
\left|I_{x y z}\right|+\left|I_{x z y}\right|+\left|I_{z x y}\right|>\left|I_{z y x}\right|+\left|I_{y x z}\right|+\left|I_{y z x}\right| .
\end{gathered}
$$

To see that CON implies MEPD, observe that whenever $y$ is supported by a majority against $x$, and $x$ Pareto dominates $z, y$ will also be supported by a majority against $z$ and should therefore be chosen according to CON. ${ }^{18}$

Another way of seeing the connection between our three axioms that formalize the idea of majoritarianism is the following. With three candidates, the existence of a Condorcet-winner implies the existence of a (weak) Condorcet-loser, i.e., of a candidate that is not majority-preferred over either of the other two. Hence, we can equivalently define CON as ensuring the election of the majority preferred Candidate after the elimination of a Condorcet-loser-rather than the elimination of a Paretodominated candidate as under MEPD or a universally least-preferred candidate as under MEW.

Figure 1 presents the logical relations between the axioms described so far. Note that they are all intra-profile axioms, i.e., they all concern the behaviour of a social choice correspondence within given preference profiles.

Our final axioms concerns its behaviour across profiles. For that, consider an arbitrary profile $\succ_{I}=\left(\succ_{i}\right)_{i \in I}$. For any $x \in A$, another profile $\succ_{I}^{\prime}=\left(\succ_{i}^{\prime}\right)_{i \in I}$ is said to be an $x$-monotonic transformation of $\succ_{I}$, iff

$$
\begin{aligned}
\forall i \in I, y, z \neq x: \quad x \succ_{i} y \Longleftrightarrow x \succ_{i}^{\prime} y, \quad x \succ_{i} z \Longrightarrow x \succ_{i}^{\prime} z, \text { and } \\
y \succ_{i} z \Longleftrightarrow y \succ_{i}^{\prime} z,
\end{aligned}
$$

i.e., such that $x$ is more popular under $\succ_{I}^{\prime}$, while the ordering of $y$ and $z$ remains unchanged. If $x$ is the unique solution under $\succ_{I}$, it should remain so under $\succ_{I}^{\prime}$.

Definition 7 A scoring rule $V$ is said to satisfy Monotonicity (MON), if for any preference profile $\succ_{I}$ and an $x$-monotonic transformation $\succ_{I}^{\prime}$ we have

$$
\{x\}=S\left(\succ_{I}, V\right) \Longrightarrow\{x\}=S\left(\succ_{I}^{\prime}, V\right) .
$$

Alternatively one may require, that if $x$ was included in the solution for $\succ_{I}$, it should remain so for $\succ_{I}^{\prime}$.

\footnotetext{
18 If instead $x$ is majority-preferred over $y$, and Pareto-dominates $z, x$ is all the more easily recognized as Condorcet-winner and should hence be chosen according to $\mathbf{C O N}$.
} 
Fig. 2 Logical relations between cross-profile, monotonicity axioms

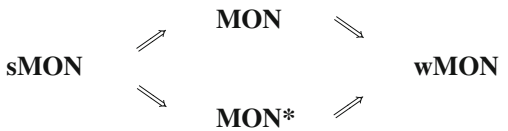

Definition 8 A scoring rule $V$ is said to satisfy Monotonicity* (MON*), if for any preference profile $\succ_{I}$ and an $x$-monotonic transformation $\succ_{I}^{\prime}$ we have

$$
x \in S\left(\succ_{I}, V\right) \Longrightarrow x \in S\left(\succ_{I}^{\prime}, V\right) .
$$

Note that both notions are logically independent-while MON rests on a stronger premise, MON* has a weaker implication. Both are implied by the following stronger notion resembling "positive responsiveness" by which May characterized majority rule in the two alternative setting (May 1952). ${ }^{19}$

Definition 9 A scoring rule $V$ is said to satisfy strong Monotonicity (sMON), if for any preference profile $\succ_{I}$ and an $x$-monotonic transformation $\succ_{I}^{\prime}$ we have

$$
x \in S\left(\succ_{I}, V\right) \Longrightarrow\{x\}=S\left(\succ_{I}^{\prime}, V\right) \text {. }
$$

Finally, all of the above imply the following weak notion of monotonicity.

Definition 10 A scoring rule $V$ is said to satisfy weak Monotonicity (wMON), if for any preference profile $\succ_{I}$ and an $x$-monotonic transformation $\succ_{I}^{\prime}$ we have

$$
\{x\}=S\left(\succ_{I}, V\right) \Longrightarrow x \in S\left(\succ_{I}^{\prime}, V\right) .
$$

Monotonicity properties may be particularly important where candidates are engaged in electoral competition, i.e., where they can choose a policy platform and thereby affect their position in voters' rankings of candidates. A violation of MON or MON* would create perverse incentives for candidates - a candidate may then increase her chance of election by adjusting her platform only to hurt some group within the electorate, moving her down in that groups' rankings of candidates (while leaving everyone's ranking of the other candidates unchanged). For example, under a violation of MON, the candidate may be uniquely selected only after the change in platform, i.e., after she has lost in popularity.

\section{Results}

We first analyse positional scoring rules alongside all other direct mechanism scoring rules where we are in particular able to axiomatise the Borda Rule and Approval Voting. We then move on to general finite scoring rules to which we are able to generalise some

19 In the two alternative setting, MON and MON* can be shown to be equivalent. May's "positive responsiveness", resp. sMON, strengthens the axioms, essentially demanding that whenever two alternatives are reasonable choices in light of a group's preferences, that 'tie' should be broken by even the smallest increase in popularity of either. 
of our results, in particular our impossibility results. Finally, we extend our analysis further and discuss selected alternative voting procedures.

\subsection{Direct mechanism scoring rules}

Our first result maps out the class of such rules by showing that positional and votesplitting scoring rules are essentially the only direct mechanism scoring rules (cases (1) and (2)); the only other rules are slight variations of the Plurality (3) and Antiplurality Rule (4).

Lemma 1 For any direct mechanism scoring rule $V$ one of four cases applies: Up to elimination of abstensions and a normalization of ballots, the set of admissible ballots $V$ consists of

all permutations of $(1, s, 0)$, for some fixed $s \in[0,1]$.

all permutations of $(s, s, 0)$ and $(1-s, 0,0)$, for some fixed $s \in(0,1)$.

all permutations of $(1,0,0)$ and $(s, 0,0)$, for some fixed $s \in(0,1)$.

all permutations of $(1,1,0)$ and $(s, s, 0)$, for some fixed $s \in(0,1)$.

The intuition behind Lemma 1 is straightforward. Suppose $V$ contains an admissible ballot $w$ with three distinct entries. Since $V$ is neutral with respect to a relabelling of candidates, the corresponding 6 permutations of $w$ are all included in, and exhaust, $V$. Normalizing then yields case (1). If $V$ contains a ballot $w$ with two identical entries, it also contains all 3 of its permutations. This leaves room for another ballot $w^{\prime}$ which can have only 3 permutations itself, i.e., must contain two identical entries as well. Normalizing $w$ and $w^{\prime}$, as well as their permutations yields one of the cases (2)-(4). A slightly more formal proof is found in the Appendix.

Having delineated the set of direct mechanisms under consideration, we now turn to their strategic analysis by iterative elimination of weakly dominated strategies. For that we will make use of the following useful fact.

Fact 1 In approval voting games, the set of undominated strategies for voter $i \in I_{x y z}$ consists of all ballots $v_{i} \in V$ for which $v_{i}^{x}=1 / 2$ and $v_{i}^{z}=0$, i.e., where she approves of her most but not of her least preferred candidate (Brams and Fishburn 1978). In positional scoring rule voting games, undominated strategies of $i \in I_{x y z}$ are all ballots $v_{i} \in V$ for which $v_{i}^{x} \geq s \geq v_{i}^{z}$, i.e., that award a weakly higher score to her most than to her least preferred candidate (Buenrostro et al. 2013, Proposition 1).

Using Fact 1, we will show that the Borda Rule occupies a particularly prominent position within the class of direct mechanism scoring rules. For that, we first delineate its solution in iteratively dominated strategies.

Theorem 1 Consider a Borda Rule voting game $\Gamma\left(\succ_{I}, V^{0}\right)$. A candidate $x \in A$ is the unique solution, i.e., $S\left(\succ_{I}, V\right)=\{x\}$, if and only if we can label candidates so that 
one of the following three conditions is satisfied: Iselectfont

$$
\begin{aligned}
&\left|I_{x y z}\right|>\left|I_{x z y}\right|+\left|I_{y x z}\right|+2\left|I_{y z x}\right|+2\left|I_{z x y}\right|+2\left|I_{z y x}\right|, \\
& \text { or }\left|I_{x y z}\right|>\left|I_{x z y}\right|+\left|I_{y x z}\right|+2\left|I_{y z x}\right|+2\left|I_{z x y}\right|+2\left|I_{z y x}\right|-1 \text { and }\left|I_{z x y}\right|>\left|I_{y x z}\right|, \\
& \text { or }\left|I_{x y z}\right|>\left|I_{x z y}\right|+\left|I_{y x z}\right|+2\left|I_{y z x}\right|+2\left|I_{z x y}\right|+2\left|I_{z y x}\right|-2 \text { and }\left|I_{x z y}\right|>0 \text {. }
\end{aligned}
$$

Otherwise, no candidate can be excluded as a winner, i.e., $S\left(\succ_{I}, V\right)=A$.

The proof for the if part is similar in all three cases. We first show that either $y$ or $z$ can be ruled out as an element of $S\left(\succ_{I}, V\right)$, as after a few rounds of eliminating dominated strategies we have $v^{x}>v^{y}$ or $v^{x}>v^{z}$. Then, the choice comes down to $x$ and one other remaining candidate, and $x$ wins, as it is majority supported. We present the proof for case (1) here, and relegate cases (2) and (3) to the Appendix.

Assume (1) holds. After eliminating dominated strategies, Fact 1 implies

$$
\min _{v \in V^{1}} v^{x}-v^{z}=1 / 2\left|I_{x y z}\right|-1 / 2\left|I_{x z y}\right|-1 / 2\left|I_{y x z}\right|-\left|I_{y z x}\right|-\left|I_{z x y}\right|-\left|I_{z y x}\right|>0,
$$

so that $z$ is ruled out as an outcome. Then, in the game $\Gamma\left(\succ_{I}, V^{1}\right)$, for any voter $i$ who prefers $x$ over $y, v_{i}=\left(v_{i}^{x}, v_{i}^{y}, v_{i}^{z}\right)=\left(1,0, \frac{1}{2}\right)$ is a dominant strategy as it maximizes the impact that $i$ has on $v^{x}-v^{y}$. Eliminating all other strategies of $i$ (be they dominated by or redundant to $v_{i}$ ), we find that $x$ is the unique outcome for all remaining strategy profiles $v$, as by condition (1)

$$
v^{x} \geq\left|I_{x y z}\right|+\left|I_{x z y}\right|+\left|I_{z x y}\right|>\left|I_{y x z}\right|+\left|I_{y z x}\right|+\left|I_{z y x}\right| \geq v^{y} .
$$

This proves the if part for case (1); see Appendix for cases (2) and (3).

For an intuition behind the only if part, observe that using undominated strategies, a voter $i \in I_{x y z}$ will always award a higher score to $x$ than to $z$, a voter in $I_{z y x}$ a higher score to $z$ than to $x$, while other voters may award a higher score to either. Hence, unless $I_{x y z}$ or $I_{z y x}$ are too large relative to the other groups, we can construct a profile where $x$ and $z$ have the same score. Further, if (1)-(3) are violated under every labelling of candidates, so that no group is particularly large relative to the other groups, we are able to construct profiles of undominated strategies for which not only two but all three candidates are tied and thus any outcome is possible. Moreover, the fact that at such a profile a voter's most preferred candidate is a possible outcome often ensures that her current strategy survives further elimination of dominated strategies. ${ }^{20}$ As the construction of such strategy profiles requires a large number of case distinctions, the proof is relegated to the appendix.

In terms of our axioms, Theorem 1 has an immediate corollary.

Corollary 1 The Borda Rule satisfies both $\mathbf{U}$ and MEW.

Proof Assume that $a$ is unanimously preferred, i.e., $I=I_{a b c} \cup I_{a c b}$. Without loss of generality, we can assume $\left|I_{a b c}\right| \geq\left|I_{a c b}\right|$. If $\left|I_{a b c}\right|>\left|I_{a c b}\right|, a$ is the unique solution by Theorem 1, condition (1). If $\left|I_{a b c}\right|=\left|I_{a c b}\right|$, then $\left|I_{a c b}\right|>0$ and condition (3) holds.

20 See Claim 1 in the Proof of Theorem 1. 
Assume on the other hand that $c$ is unanimously considered the worst alternative, i.e., $I=I_{a b c} \cup I_{b a c}$, and that $a$ is majority preferred over $b$, i.e., $\left|I_{a b c}\right|>\left|I_{b a c}\right|$. Then $a$ is the unique solution by Theorem 1, condition (1).

Corollary 1 overlaps with results in Buenrostro et al. (2013) who provide sufficient conditions for scoring rule voting games to be dominance solvable, i.e., have a unique solution in iteratively undominated strategies. The corollary extends beyond their Theorems 1 and 2, in that it includes the case $I=I_{a b c} \cup I_{a c b},\left|I_{a b c}\right|=\left|I_{a c b}\right|$, i.e., implies that a unanimously preferred candidate $a$ is the unique solution even if the electorate is split in half. What might be more remarkable though, is the exceptional role among positional scoring rules that Corollary 1 grants to the Borda Rule:

Theorem 2 The Borda Rule is the unique positional scoring rule that satisfies $\mathbf{U}$ and MEW. In particular, positional scoring rules with $s<\frac{1}{2}$ violate $\mathbf{U}$, while positional scoring rules with $s>\frac{1}{2}$ violate MEW.

The proof can be found in the Appendix. For an intuition behind Theorem 2, assume that $s<1 / 2$ and that alternative $a$ is unanimously preferred. If the electorate is split in half between the groups $I_{a b c}$ and $I_{a c b}$ and every voter supports their second best alternative by awarding it a score of one, $a$ receives an average score of at most $s<1 / 2$ while $b$ and $c$ will be tied with an average score of $1 / 2$. For a large electorate, an individual who deviates and supports $a$ would then hand the election to their least preferred candidate. Hence, for each voter, supporting their second best alternative is the best response and survives the iterative elimination of weakly dominated strategies-which establishes both $b$ and $c$ as element of the solution $S\left(\succ_{I}, V\right)$.

Similarly, assume that $s>1 / 2$ and everyone agrees that $c$ is the worst alternative. If the groups $I_{a b c}$ and $I_{b a c}$ are almost of same size, it is possible that $a$ and $b$ receive almost the same score so that a single voter is pivotal. In such a situation, awarding a score of $s$ to the least preferred alternative $c$-and a score of zero to the second best alternative - may be the best response, as it tips the election in favour of the most preferred alternative. Yet, if awarding a score of $s$ to $c$ is not dominated and hence cannot be ruled out, $c$ may win with an average score of $s>1 / 2$ while $a$ and $b$ have an average score of about $1 / 2$.

In light of Theorem 2, it is natural to ask whether there exist other direct mechanism scoring rules, beyond the Borda Rule, that simultaneously satisfy $\mathbf{U}$ and MEW. The most prominent direct mechanism scoring rule not covered by Theorem 2 is Approval Voting, for which Courtin and Núñez (2017) provide necessary-and-sufficient conditions for the associated voting games to be dominance solvable, i.e., to have a unique solution in iteratively undominated strategies: ${ }^{21}$

Fact 2 Let $\Gamma\left(\succ_{I}, V^{0}\right)$ be an Approval voting game and let $S\left(\succ_{I}, A V\right)$ denote its solution. It is unique, i.e., $S\left(\succ_{I}, A V\right)=\{x\}, x \in A$, if and only if we can label

\footnotetext{
21 For the sake of completeness and since our the formal treatment of ties is different from Courtin and Núñez (2017), we reproduce their result in the appendix.
} 
candidates so that both of the following two conditions are satisfied:

$$
\begin{aligned}
& \left|I_{x y z}\right|>\left|I_{y z x}\right|+\left|I_{z x y}\right|+\left|I_{z y x}\right| \text { or } \quad\left|I_{y x z}\right|>\left|I_{x z y}\right|+\left|I_{z x y}\right|+\left|I_{z y x}\right| \\
& \text { and }\left|I_{x y z}\right|+\left|I_{x z y}\right|+\left|I_{z x y}\right|>\left|I_{z y x}\right|+\left|I_{y x z}\right|+\left|I_{y z x}\right| .
\end{aligned}
$$

The first condition rules out some $z$ as an outcome, as either $x$ or $y$ have a strictly higher score in undominated strategies; the second demands that $x$ is majority-preferred over $y$. If only (1) is satisfied while the electorate is split, one half preferring $x$ over $y$, the other $y$ over $x$, then $S\left(\succ_{I}, A V\right)=\{x, y\}$. If (1) is not satisfied under any labelling of candidates, then $S\left(\succ_{I}, A V\right)=A$.

Fact 2 reveals that Approval Voting satisfied wMON-if initially $z$ could be ruled out based on the condition (1) and $x$ was majority preferred over $y$, so that $x$ was the unique solution, then either the same holds after $x$ gains in popularity, or no candidate is ruled out based on (1), so that any candidate, including $x$, may win.

Moreover, AV satisfies not only $\mathbf{U}$ and MEW but even the stronger, unifying axiom of being majoritarian after eliminating a Pareto-dominated candidate. As we will see, it is the only direct mechanism scoring rule that satisfies it.

Theorem 3 Approval Voting is the unique direct mechanism scoring rule that satisfies MEPD. In particular, vote-splitting scoring rules with $s<\frac{1}{2}$ and scoring rules where $V$ consists of all permutations of $(1,0,0)$ and $(s, 0,0), s \in(0,1)$, violate $\mathbf{U}$, while vote-splitting scoring rules with $s>\frac{1}{2}$ and scoring rules where $V$ consists of all permutations of $(1,1,0)$ and $(s, s, 0), s \in(0,1)$, violate MEW.

The fact that the Borda Rule, while satisfying $\mathbf{U}$ and MEW, fails to satisfy MEPD, follows from Theorem 1. For example, consider a preference profile $\succ_{I}$ where $I=$ $I_{a b c} \cup I_{a c b} \cup I_{b a c}$ and $\left|I_{a b c}\right|=\left|I_{a c b}\right|=\left|I_{b a c}\right|=n \geq 2$. Then MEPD requires $a$ to be the unique solution, while by Theorem 1 we have $S\left(\succ_{I}, V\right)=A$. All other positional scoring rules violate either $\mathbf{U}$ or MEW and hence also MEPD, see Theorem 2.

To see that Approval Voting satisfies MEPD, consider a preference profile where $a$ Pareto-dominates $c$, i.e., where $I=I_{a b c} \cup I_{a c b} \cup I_{b a c}$. After eliminating dominated strategies, each voter awards a weakly higher score to $a$ than to $c$ (Fact 1), so that for any $v \in V^{1}$, we have $v^{a} \geq v^{c}$.

Moreover, if there exists a voter $i \in I_{a b c}$, she votes either $(1 / 2,1 / 2,0)$ or $(1 / 2,0,0)$, ensuring that $v^{a}>v^{c}$ and ruling out outcome $c$ after one round of elimination. In the next step, each voter awards a score of $1 / 2$ to her preferred among the remaining candidates $a$ and $b$, and a score of zero to the other. Hence the majority-preferred candidate wins after two rounds of elimination of dominated strategies.

If on the other hand $\left|I_{a b c}\right|=0$, so that $I=I_{a c b} \cup I_{b a c}$, we have to consider two cases. First consider $\left|I_{a c b}\right|>\left|I_{b a c}\right|$, i.e., $a$ is majority-preferred over $b$. Then, for any $v \in V^{1}$, we have $v^{a} \geq \frac{\left|I_{a c b}\right|}{2}>\frac{\left|I_{b a c}\right|}{2}=v^{b}$ so that $b$ is ruled out as an outcome. In the next step, no voter approves of $c$, so that $a$ is the only outcome after two rounds of elimination.

Finally, if $I=I_{a c b} \cup I_{b a c}$ and $\left|I_{b a c}\right|>\left|I_{a c b}\right|$, we know that for any $v \in V^{1}$, $v^{b}=\frac{\left|I_{b a c}\right|}{2}>\frac{\left|I_{a c b}\right|}{2} \geq v^{c}$, so that $c$ is ruled out as an outcome. In the next step, no voter $i \in I_{b a c}$ approves of $a$, so that the majority-preferred candidate $b$ wins. 
It remains to show that no other vote-splitting scoring rule, and no direct mechanism scoring rules of type (3) or (4) in Lemma 1, satisfies MEPD. For that, the reader is referred to the Appendix.

We are now left with only two direct mechanism scoring rules that satisfy $\mathbf{U}$ and MEW, namely the Borda Rule and Approval Voting where only the latter satisfies the even stronger axiom MEPD. With respect to our monotonicity axioms, Approval Voting satisfies wMON but fails both stronger notions of monotonicity, MON and MON*, as can be seen in the following example.

Example 1 Consider a preference profile $\succ_{I}$ where $I=I_{a b c} \cup I_{b a c} \cup I_{c a b}$ and

$$
\left|I_{a b c}\right|=2, \quad\left|I_{b a c}\right|=4, \quad\left|I_{c a b}\right|=3 .
$$

After eliminating dominated strategies, it is clear that $b$ will have a score of at least $\frac{\left|I_{b a c}\right|}{2}=2$, while the score of $c$ is equal to $\frac{\left|I_{c a b}\right|}{2}<2$ (see Fact 1 ). This reduces the game further, to an election between $a$ and $b$, which $a$ wins with a score of $v^{a}=\frac{\left|I_{a b c}\right|+\left|I_{c a b}\right|}{2}=\frac{5}{2}>2=\frac{\left|I_{b a c}\right|}{2}=v^{b}$. Hence we have $S\left(\succ_{I}^{\prime}, V\right)=\{a\}$ as the unique solution in iteratively undominated strategies.

But if $a$ increases in popularity, so that we now have $\succ_{I}^{\prime}$ with $I=I_{a b c}^{\prime} \cup I_{b a c}^{\prime} \cup I_{c a b}^{\prime}$ and $\left|I_{a b c}^{\prime}\right|=\left|I_{b a c}^{\prime}\right|=\left|I_{c a b}^{\prime}\right|=3$, a three-way tie at a score of $\frac{3}{2}$ is possible if each voter approves of their most-preferred candidate only. As this makes the election of any voter's most-preferred candidate a possible outcome and since also approving of the second-most-preferred candidate rules out that possibility for any tiebreaker, approving of the most-preferred candidate only is an undominated strategy for each voter. Hence, we have $S\left(\succ_{I}^{\prime}, V\right)=A$.

Since the Condorcet-winner $a$ is no longer the only possible outcome after increasing in popularity, we see that Approval Voting violates CON and MON. Moreover, note that as we move in the other direction, from $\succ_{I}^{\prime}$ to $\succ_{I}$, it is $b$ that is increasing in popularity and that is no longer included as a possible outcome under $\succ_{I}$. Hence, Approval Voting also violates MON*.

Example 1 not only presents a novel paradox for Approval Voting but yields two impossibility results.

Corollary 2 No social choice correspondence that satisfies both MEPD and either MON or MON* can be implemented by a direct mechanism scoring rule.

Corollary 3 No social choice correspondence that satisfies CON can be implemented by a direct mechanism scoring rule.

Both impossibility results are immediate implications of Theorem 3 and Example 1. Example 1 documents a violation of MON and MON* but also of CON by Approval Voting, while Theorem 3 establishes that all other direct mechanism scoring rules violate MEPD and hence also CON. Note that even for positional scoring rules, Corollary 3 is not implied by the well known fact that these classical rules violate Condorcetconsistency under sincere voting.

In contrast to Approval Voting, the Borda Rule satisfies at least one of our stronger notions of monotonicity-and is in fact partially characterized by it: 
Theorem 4 The Borda Rule is the unique direct mechanism scoring rule that satisfies U, MEW and MON.

Proof In light of Theorems 2 and 3 as well as Example 1, it remains to show that the Borda Rule satisfies MON. For that, assume some candidate, say $a$, is the unique solution in $\Gamma\left(\succ_{I}, V^{0}\right)$. Then we know from Theorem 1 that, up to relabelling of candidates $b$ and $c$, at least one of three conditions is satisfied:

$$
\begin{aligned}
& \quad\left|I_{a b c}\right|>\left|I_{a c b}\right|+\left|I_{b a c}\right|+2\left|I_{b c a}\right|+2\left|I_{c a b}\right|+2\left|I_{c b a}\right|, \\
& \text { or }\left|I_{a b c}\right|>\left|I_{a c b}\right|+\left|I_{b a c}\right|+2\left|I_{b c a}\right|+2\left|I_{c a b}\right|+2\left|I_{c b a}\right|-1 \text { and }\left|I_{c a b}\right|>\left|I_{b a c}\right|, \\
& \text { or }\left|I_{a b c}\right|>\left|I_{a c b}\right|+\left|I_{b a c}\right|+2\left|I_{b c a}\right|+2\left|I_{c a b}\right|+2\left|I_{c b a}\right|-2 \text { and }\left|I_{a c b}\right|>0 \text {. }
\end{aligned}
$$

As we move to an $a$-monotonic transformation of $\succ_{I}$, this

- weakly increase $\left|I_{a b c}\right|$,

- weakly decrease $\left|I_{a c b}\right|+\left|I_{b a c}\right|+2\left|I_{b c a}\right|+2\left|I_{c a b}\right|+2\left|I_{c b a}\right|$,

- and weakly relaxes the inequality $\left|I_{a c b}\right|>0$.

Hence, if initially conditions (1) or condition (3) were satisfied, they continue to hold, so that $a$ is still the unique solution. If initially only condition (2) was satisfied, the inequality $\left|I_{c a b}\right|>\left|I_{b a c}\right|$ could cease to hold when moving to an $a$-monotonic transformation of $\succ_{I}$ as $\left|I_{b a c}\right|$ increase (some $i$ moves from $I_{b c a}$ to $I_{b a c}$ ), or as $\left|I_{c a b}\right|$ shrinks (some $i$ moves from $I_{c a b}$ to $I_{a c b}$ ). But then in both cases (1) is satisfied, as $\left|I_{a c b}\right|+\left|I_{b a c}\right|+2\left|I_{b c a}\right|+2\left|I_{c a b}\right|+2\left|I_{c b a}\right|$ decreases. Hence, $a$ remains the unique solution so that MON is satisfied.

That the Borda Rule, in contrast to Approval Voting, satisfies at least one notion of monotonicity, MON, raises the question of whether it also satisfies the other, MON*. Unfortunately, the following example shows that it does not.

Example 2 Consider a preference profile $\succ_{I}$ where $I=I_{a b c} \cup I_{a c b} \cup I_{b a c}$ and $\left|I_{a b c}\right|=$ $\left|I_{a c b}\right|=\left|I_{b a c}\right|=2$. Then for any labelling of candidates we have

$$
\begin{aligned}
\left|I_{x y z}\right| & \leq\left|I_{x z y}\right|+\left|I_{y x z}\right|+\left|I_{y z x}\right|+\left|I_{z x y}\right|+\left|I_{z y x}\right|-2 \\
& \leq\left|I_{x z y}\right|+\left|I_{y x z}\right|+2\left|I_{y z x}\right|+2\left|I_{z x y}\right|+2\left|I_{z y x}\right|-2
\end{aligned}
$$

so that by Theorem 1 we have $S\left(\succ_{I}, V\right)=A{ }^{22}$ But if $b$ increases in popularity, so that we now have $\succ_{I}^{\prime}$ with $\left|I_{a b c}^{\prime}\right|=3,\left|I_{a c b}^{\prime}\right|=1$ and $\left|I_{b a c}^{\prime}\right|=2$, then condition (3) of Theorem 1 is satisfied (for $x=a, y=b$ and $z=c$ ) and $S\left(\succ_{I}^{\prime}, V\right)=\{a\}$. Hence $b$ is no longer included after increasing in popularity, a violation of MON*.

Example 2 yields an impossibility result for direct mechanism scoring rules.

Corollary 4 No social choice correspondence that satisfies $\mathbf{U}$, MEW, and MON* can be implemented by a direct mechanism scoring rule.

This follows from the fact that $\mathbf{U}$ and MEW narrow the set of rules down to the Borda Rule and Approval Voting, neither of which satisfies MON*.

\footnotetext{
22 See case 3.1 in the Proof of Theorem 1 to see explicitly how the process of iterative elimination of strategies comes to a halt before any alternative can be excluded.
} 


\subsection{Finite scoring rules}

Let us now consider finite scoring rules, i.e., scoring rules where the number of admissible ballots can be any natural number, $|V| \in \mathbb{N}$. Two well known examples are Evaluative Voting, where every voter $i$ assigns an individual score $v_{i}^{x} \in\{0,1, \ldots, m\}$ to each candidate $x$, and Cumulative Voting, differing from Evaluative Voting in that there is a total budget of $m$ points that may, or must, be distributed among candidates. To avoid trivial cases, we assume that $V$ contains at least one non-abstention ballot and assume without loss of generality that ballots are normalized such that (i) on every ballot, the lowest score awarded to some candidate is zero, ${ }^{23}$ and (ii) the highest score awarded to some candidate on some ballot is one. ${ }^{24}$

Hence, under this normalization, every finite scoring rule allows to cast ballots $w \in V$ that are, up to permutations, of the form $\left(k_{w}, l_{w}, 0\right)$ with $1 \geq k_{w} \geq l_{w} \geq$ 0 and where $k_{w}=1$ for at least one $w \in V$. Approval Voting corresponds to a set of admissible ballots $V$ that consists of $(1,0,0)$ and $(1,1,0)$ as well as their permutations. ${ }^{25}$ Intuitively, by casting ballot $(1,0,0)$, a voter provides the strongest support to one candidate (against the two others) that is conceivable under any finite scoring rule, while by casting ballot $(1,1,0)$ a voter raises the strongest possible opposition against one candidate. We refer to a finite scoring rule where $V$ includes both these 'extreme' ballots as an Extended Approval Voting Rule.

Definition 11 A finite scoring rule $V$ is said to be an Extended Approval Voting Rule (EAV-rule), if $\{(1,0,0),(1,1,0)\} \subset V$.

A prime example of an Extended Approval Voting Rule is Evaluative Voting; Cumulative Voting on the other hand does not fall within this class, as it allows a voter to assign the highest possible score to one candidate only when all other candidates receive a score of zero. As we will see, EAV-rules will share a number of interesting properties by virtue of all sharing the extremal ballots of Approval Voting. ${ }^{26}$ For one, they all engender sincere voting:

Lemma 2 Let $V$ be an EAV-rule and consider the induced voting game $\Gamma\left(\succ_{I}, V^{0}\right)$. Then for any voter $i$, any undominated strategy $v_{i} \in V_{i}^{1}$ is either a sincere strategy (i.e., assigns weakly higher scores to more preferred candidates) or redundant to a sincere strategy in $\Gamma\left(\succ_{I}, V^{0}\right)$.

Proof Without loss of generality, let us assume that $i$ 's preferences are $a \succ_{i} b \succ_{i} c$. For $v_{i} \in V$ such that $v_{i}^{a}<v_{i}^{b}$, we claim that $v_{i}$ is dominated by, or redundant

\footnotetext{
${ }^{23}$ Otherwise, for each $v_{i} \in V$, take $u=\min _{x \in A} v_{i}^{x}$ and replace $v_{i}$ by $v_{i}^{\prime}=\left(v_{i}^{a}-u, v_{i}^{b}-u, v_{i}^{c}-u\right)$.

24 Otherwise, set $u=\max _{x \in A, v_{i} \in V} v_{i}^{x}$ and replace each $v_{i}$ by $v_{i}^{\prime}=\left(\frac{v_{i}^{a}}{u}, \frac{v_{i}^{b}}{u}, \frac{v_{i}^{c}}{u}\right)$.

25 This differs from the normalization in Sect. 4.1 where Approval Voting allowed for ballots $(1 / 2,0,0)$ and $(1 / 2,1 / 2,0)$.

26 Núñez and Laslier (2014) compare the sets of equilibria of different voting rules with common extremal ballots (in large electorate Poisson voting games) defining extremal ballots as those non-abstention ballots that cannot be derived as a strict convex combination of other admissible ballots. On can show that, by this definition, all EAV rules that allow for abstention have the same extremal ballots-namely $(1,0,0),(1,1,0)$ as well as permutations thereof-and are hence strategically equivalent in the setting of Núnez and Laslier (2014) (see their Theorem 1).
} 
to, $\tilde{v}_{i}=(1,1,0)$. To see this, note that for any $v_{-i}$ and $\tilde{v}=\left(\tilde{v}_{i}, v_{-i}\right)$ we have $\tilde{v}^{a}-\tilde{v}^{b}>v^{a}-v^{b}$ and $\tilde{v}^{b}-\tilde{v}^{c} \geq v^{b}-v^{c}$. Hence, if under $v$ we had $a$ as a possible winner (possibly tied and depending on $\triangleright$ ), then under $\tilde{v}$ it is the unique winner. If $b$ was the unique winner under $v, \tilde{v}$ still rules out $c$ but may yield $a$ as possible outcome; if under $v$ we had a tie of $b$ and $c$ and we have a different outcome under $\tilde{v}$, then either $c$ is ruled out, or $a$ becomes a possible outcome (or both); if $c$ was the unique winner, $\tilde{v}$ can only yield a weakly better outcome - in all cases, and for any $\triangleright, \tilde{v}$ yields a weakly better outcome for voter $i$. Hence, $v_{i}$ is either weakly dominated by $\tilde{v}_{i}$, or, if it yields the same outcome against any $v_{-i}$ and $\triangleright$, is redundant to it. In the same way, one shows that any $v_{i}$ with $v_{i}^{b}<v_{i}^{c}$ is dominated by, or redundant to, $\tilde{v}_{i}=(1,0,0)$.

Using Lemma 2, it is straightforward to show that all EAV-rules satisfy MEPD. In fact the converse holds as well - the axiom characterizes the set of EAV-rules:

\section{Theorem 5 A finite scoring rule satisfies MEPD if and only if it is an EAV-rule.}

The if part rests on Lemma 2. Without loss of generality, assume $V^{1}$ contains sincere strategies only (otherwise, eliminate all redundant insincere strategies and arrive at $\tilde{V}^{1}$ for which this is the case). Then in undominated strategies, every voter awards a weakly lower score to a Pareto-dominated candidate, say $c$, than to the candidate dominating it, say $a$. Moreover, if there exists a voter $i \in I_{a b c}$, she awards a strictly higher score to $a$ than to $c$, ruling out the election of $c$. Hence, in the next step, each voter awards a score of 1 to her preferred candidate among $a$ and $b$, and a score of zero to the other, so that the majority-preferred candidate wins.

If there is no voter $i \in I_{a b c}$ then $I=I_{a c b} \cup I_{b a c}$. A voter $i \in I_{a c b}$ in $\Gamma\left(\succ_{I}, V^{1}\right)$ awards a weakly higher score to $a$ than to $c$. Among those strategies that award the same score to $a$ and $c, v_{i}=(1,0,1)$ dominates (or is redundant to) any other, as it maximizes the score difference between $a, c$ and the least preferred candidate $b$. If instead $i \in I_{a c b}$ awards a strictly higher score to $a$ than to $c$, this rules of the election of $c$ - so that among these strategies, $v_{i}=(1,0,0)$ dominates (or is redundant to) any other. Hence, after removing dominated (and possibly redundant) strategies from $V_{i}^{1}$, all $i \in I_{a c b}$ award a score of 1 to $a$ and a score of 0 to $b$. Now, if $\left|I_{a c b}\right|>\left|I_{b a c}\right|$, this implies $v^{a}-v^{b} \geq\left|I_{a c b}\right|-\left|I_{b a c}\right|>0$ which rules out $b$ as an outcome. In the next step, each voter assigns a score of 1 to $a$ and a score of 0 to $c$ so that $a$ is the unique winner.

Finally, if instead $I=I_{a c b} \cup I_{b a c}$ and $\left|I_{a c b}\right|<\left|I_{b a c}\right|$, consider a voter $i \in I_{b a c}$. Among those strategies that assign the same score to $a$ and $c$, namely zero, $v_{i}=$ $(0,1,0)$ is dominant and if all $i \in I_{a b c}$ follow that strategy then $b$ is elected uniquely. If instead at least one voter $i \in I_{b a c}$ assigns a positive score to $a$, then this again rules out the election of $c$. Hence, $c$ may never be elected in $\Gamma\left(\succ_{I}, V^{1}\right)$, so that all $i \in I_{b a c}$ will, after eliminating dominated (and possibly redundant) strategies from $V_{i}^{1}$ choose $v_{i}=(0,1,0)$ - which elects $b$.

For the only if part, we have to show that any non-EAV-rule $V$ fails MEPD. Recall that by our normalization, each admissible ballot assigns a score of zero to some candidate and some ballot assigns a score of one to some candidate. Define

$$
\underline{s}=\min \{s \mid(1, s, 0) \in V)\} \quad \text { and } \quad \bar{s}=\max \{s \mid(1, s, 0) \in V)\} .
$$


Since $V$ is not an EAV-rule, $\underline{s}>0$ or $\bar{s}<1$. We first consider $\underline{s}+\bar{s}<1$ with $\underline{s}$ and $\bar{s}$ rational. We will show that for some preference profile $\bar{I}=I_{a b c} \cup I_{b c a}$ with $\left|I_{a b c}\right|<\left|I_{b c a}\right|$ the iterative elimination of dominated strategies cannot narrow down the set of strategies beyond $V_{a b c}^{\prime \prime}=\{(\bar{s}, 1,0),(1,0, \underline{s}),(1, \underline{s}, 0)\}$ and $V_{b c a}^{\prime \prime}=$ $\{(0, \bar{s}, 1),(0,1, \bar{s}),(\underline{s}, 1,0)\}$ so that in particular the Pareto-dominated candidate $c$ may win the election.

For that, consider $\left|I_{a b c}\right|=n_{1}+n_{2}+n_{3}$ and $\left|I_{b c a}\right|=m+n_{1}+n_{2}+n_{3}$ for some $m, n_{1}, n_{2}, n_{3} \in \mathbb{N}$ and $0<m \leq n_{2}+n_{3}$. Construct $v$ as follows.

- $m$ of $I_{a b c}$ choose $v_{i}=(1,0, \underline{s})$

- $n_{1}$ of $I_{a b c}$ choose $v_{i}=(\bar{s}, 1,0)$

- $n_{2}+n_{3}-m$ of $I_{a b c}$ choose $v_{i}=(1, \underline{s}, 0)$

- $m$ of $I_{b c a}$ choose $v_{i}=(\underline{s}, 1,0)$

- $n_{1}$ of $I_{b c a}$ choose $v_{i}=(0,1, \bar{s})$

- $n_{2}+n_{3}$ of $I_{b c a}$ choose $(0, \bar{s}, 1)$

Then $v^{a}=v^{c}=m \cdot \underline{s}+n_{1} \cdot \bar{s}+n_{2}+n_{3}, v^{b}=m(1-\underline{s})+2 n_{1}+\left(n_{2}+n_{3}\right)(\underline{s}+\bar{s})$ and hence

$$
v^{a, c}-v^{b}=-m \underbrace{(1-2 \underline{s})}_{>0}-n_{1} \underbrace{(2-\bar{s})}_{>0}+\left(n_{2}+n_{3}\right) \underbrace{(1-\underline{s}-\bar{s})}_{>0} .
$$

Since $\underline{s}, \bar{s} \in \mathbb{Q}$, we can find $m, n_{1}, n_{2}, n_{3}>M$, for any given $M$, such that $-m(1-$ $2 \underline{s})+n_{2}(1-\underline{s}-\bar{s})=0$ and $-n_{1}(2-\bar{s})+n_{3}(1-\underline{s}-\bar{s})=0$. Moreover, $n_{3}$ can be chosen large enough (together with $n_{1}$ ) such that $n_{2}+n_{3}>m$. Denote the set of tuples $\left(m, n_{1}, n_{2}, n_{3}\right) \in \mathbb{N}^{4}$ such that these conditions are satisfied as $P^{M}$.

At any $v$ with $\left(m, n_{1}, n_{2}, n_{3}\right) \in P^{M}$, all three candidates are tied. Hence $a$ would be elected for $a \triangleright b, c$ while a switch away from $v_{i}=(1, \underline{s}, 0) \in V_{a b c}^{\prime \prime}$ to some other strategy precludes the election of $a$. Thus, $v_{i}=(1, \underline{s}, 0) \in V_{a b c}^{\prime \prime}$ is undominated in any game $\Gamma\left(\succ_{I}, V^{\prime}\right), V^{\prime \prime} \subseteq V^{\prime} \subseteq V^{1}$. In the same way, $(1,0, \underline{s}) \in V_{a b c}^{\prime \prime}$ and $(\underline{s}, 1,0) \in V_{b c a}^{\prime \prime}$ are seen to be undominated in any such game $\Gamma\left(\succ_{I}, V^{\prime}\right)$. Moreover, $(0,1, \bar{s}) \in V_{b c a}^{\prime \prime}$ is undominated as a deviation that reduced the score difference of $a$ and $b$ would again rule out the election of the most-preferred candidate, while a switch to some other strategy $(0,1, s), s<\bar{s}$, would change the outcome from $c$ to $a$ given $c \triangleright a \triangleright b$.

To see how $(\bar{s}, 1,0) \in V_{a b c}^{\prime \prime}$ may likewise be undominated as long as the strategies used in the construction of $v$ are present, construct $\tilde{v}$ from $v$ by letting $k$ of $I_{a b c}$ switch from $(1, \underline{s}, 0)$ to $(\bar{s}, 1,0)$ and $l$ of $I_{b c a}$ from $(0,1, \bar{s})$ to $(0, \bar{s}, 1)$. Then $\tilde{v}^{b}-\tilde{v}^{c}=$ $k(1-\underline{s})-l 2(1-\bar{s})$. With $\underline{s}$ and $\bar{s}$ rational, we can find $k$ and $l$ such that $\tilde{v}^{b}-\tilde{v}^{c}=0$. Moreover, choosing $k$ and $\bar{l}$ large enough, we have $\tilde{v}^{c}-\tilde{v}^{a}>2$ and setting $M>k, l$ ensure that for $v \in P^{M}$, the construction of $\tilde{v}$ is well defined. Then a voter $i \in I_{a b c}$ who at $\tilde{v}$ uses strategy $v_{i}=(\bar{s}, 1,0)$ would preclude the election of $b$, and make $c$ the unique winner if they deviated to any other strategy.

To see how $(0, \bar{s}, 1) \in V_{b c a}^{\prime \prime}$ may likewise be undominated, construct $\hat{v}$ from $v$ by letting $k^{\prime}$ of $I_{a b c}$ shift from $(\bar{s}, 1,0)$ to $(1, \underline{s}, 0)$ and $k^{\prime}$ of $I_{b c a}$ shift from $(0,1, \bar{s})$ to $(0, \bar{s}, 1)$. Then $a$ and $c$ are still tied and $\hat{v}^{c}-\hat{v}^{b}=k^{\prime}(3-\underline{s}-2 \bar{s})$. With $k^{\prime}$ large enough $\hat{v}^{c}-\hat{v}^{b}>2$ and setting $M>k^{\prime}$ ensure that for $v \in P^{M}$, the construction of $\tilde{v}$ is well 
defined. Then a voter $i \in I_{b c a}$ who at $\hat{v}$ uses strategy $v_{i}=(0, \bar{s}, 1)$ would preclude the election of $c$, and make $a$ the unique winner, if they deviated to any other strategy.

Hence, for a preference profile $I=I_{a b c} \cup I_{b c a}$ with $\left|I_{a b c}\right|=n_{1}+n_{2}+n_{3}$, $\left|I_{b c a}\right|=m+n_{1}+n_{2}+n_{3}$ such that $\left(m, n_{1}, n_{2}, n_{3}\right) \in P^{M}, M>k, l, k^{\prime}$, no strategy used in the construction of $v$ can be eliminated while the others are present. Thus they jointly survive the process of iterative elimination and $c$ is never eliminated as an outcome, violating MEPD. For the remaining cases $\underline{s}+\bar{s}>1$ and $\underline{s}+\bar{s}=1$ see the Appendix. There we also generalize the above argument and allow $\underline{s}, \bar{s}$ to be irrational, which forces us to consider near-ties and necessitates further case distinctions.

A natural next question, in light of Theorem 5 and the violations of monotonicity and CON by Approval Voting documented in Example 1, is whether any EAV-rule is monotonic or satisfies not only MEPD but even CON. The following example provides a negative answer.

Example 3 Consider an EAV-rule $V$ and a preference profile $\succ_{I}$ where, $I=I_{a b c} \cup$ $I_{b a c} \cup I_{b c a} \cup I_{c b a}$ and $\left|I_{a b c}\right|=m+1,\left|I_{b a c}\right|=1,\left|I_{b c a}\right|=m$ and $\left|I_{c b a}\right|=1$. Then $b$ is the Condorcet winner, majority-preferred over $a(m+2>m+1)$ and $c(2 m+2>1)$. Nonetheless, it is not the unique solution - in fact, all candidates may be tied as voters use iteratively undominated strategies. To see that, assume voters in $I_{a b c}$ approve only of $a$ (i.e., assign a score of 1 to $a$ and of zero to $b$ and $c$ ), the voter in $I_{b a c}$ approves only of $b$, voters in $I_{b c a}$ approve of both $b$ and $c$ (i.e., assign a score of 1 to each) while the voter in $I_{c b a}$ approves only of $c$. Then all candidates have a score of $m+1$. For those voters approving only of their most preferred candidate, any other strategy would rule out its election; voters in $I_{b c a}$ would make $a$ the unique winner if they assigned a lower score to both $b$ and $c$ and they would change the three-way-tie to a tie of $b$ and $a$ if they assigned a lower score to $c$-in all cases such a deviation could be harmful for some tiebreaker $\triangleright$. Hence, none of the strategies are dominated so that the set of possible winners cannot be narrowed down to eliminate $a$ or $c .^{27}$

Now, suppose $a$ gains in popularity so that we now have a preference profile $\succ_{I}^{\prime}$ where $I=I_{a b c}^{\prime} \cup I_{b a c}^{\prime} \cup I_{c b a}^{\prime}$ and $\left|I_{a b c}^{\prime}\right|=m+1,\left|I_{b a c}^{\prime}\right|=m+1$, and $\left|I_{c b a}^{\prime}\right|=1$. Since by Lemma 2 voting is sincere under any EAV, each voter awards a positive score (at least some $\varepsilon$, depending on $V$ ) to her most-preferred alternative and a score of zero to her least preferred. Then $c$ receives a score of at most 1 while for $m$ large enough $a$ and $b$ receive a larger score. This rules out $c$ and hence, in the next step, makes it dominant for all $i \in I_{a b c}^{\prime} \cup I_{b a c}^{\prime}$ to only approve of their most preferred candidate and for $i \in I_{c b a}^{\prime}$ to also approve of $b$. Hence, for $m$ large enough, the Condorcet winner $b$ is the unique winner with a score of $m+2$ against $a$ with a score of $m+1$.

The example yields two impossibility results regarding implementation in iteratively undominated strategies.

Corollary 5 No social choice correspondence that satisfies both MEPD and MON* can be implemented by a finite mechanism scoring rule.

Corollary 6 No social choice correspondence that satisfies CON can be implemented by a finite mechanism scoring rule.

\footnotetext{
27 Besides winning in a three-way tie, $a$ may also beat the Condorcet winner outright at other profiles that survive under iterative elimination.
} 
Both are immediate implications of Theorem 5 and Example 3. The example shows that any EAV-rule will violate MON* and CON, while Theorem 5 establishes that all other finite scoring rules violate MEPD and hence also CON.

Both impossibility results partially mirror, or in fact imply, our impossibility results for direct mechanism scoring rules, Corollaries 2 and 3. Whether not only MEPD and MON* but also MEPD and MON are incompatible for all finite rules remains, is a question for which we only have a partial answer: (i) non-EAV-rules violate MEPD (Theorem 5) while for (ii) EAV-rules where $V$ consists of ballots of the form $(1, s, 0)$ with $s \in[0,1]$, the same two preference profiles used in Example 1 give rise to a violation of MON. In particular, this shows MEPD and MON to be incompatible in the class of 'simple scoring rules' covered by Goertz and Maniquet (2011) or in the larger class of '(A,B)-scoring rules' covered in Myerson (2002). However, it is an open question whether the same impossibility persist for all EAV-rules, in particular when $V$ may include admissible ballots $(p, q, 0)$ awarding in total scores of less than 1 , i.e., $p+q<1$.

While we cannot rule out for now that enlarging the set of admissible ballots beyond those of Approval Voting may allow us to satisfy MON, the following result shows that, even if possible, this may only be achieved by increasing outcome multiplicity, thereby reducing the number of preference profiles at which MON has any bite. ${ }^{28}$ To be precise, consider the following definition.

Definition 12 Given two scoring rules $V$ and $V^{\prime}$, we say that $V$ is more decisive than $V^{\prime}$ if for any preference profile $\succ_{I}$ we have $S\left(\succ_{I}, V\right) \subseteq S\left(\succ_{I}, V^{\prime}\right)$.

A voting procedure is used precisely to narrow down the set of possible alternatives; hence arguably, it should narrow down the set as far as possible, subject to, e.g., anonymity, neutrality or other normative criteria which ensure that particularly desirable alternatives are included in the solution. ${ }^{29}$ In this respect Approval Voting goes farthest among all finite scoring rules satisfying MEPD - it is more decisive than any other.

Put differently, it narrows down the set of possible outcomes to those that are included under every such rule. If one insists on MEPD, and thus selects an EAV-rule, Approval Voting is the most innocuous choice-it never yields an outcome that would not also have been a possible outcome under any other such rule.

Theorem 6 Among all finite scoring rules satisfying MEPD, Approval Voting is the most decisive: for any profile $\succ_{I}$ and any EAV-rule $V$, we have $S\left(\succ_{I}, A V\right) \subseteq S\left(\succ_{I}, V\right)$.

Proof Consider any preference profile $\succ_{I}$ and any EAV-rule $V$. For any $z \in A$, let $M(z)$ denote the number of voters for whom $z$ is the most, and $S M(z)$ the number of those for whom it is the second-most preferred candidate. Recall conditions (1) and (2) of Fact 2, Sect. 4.1, describing $S\left(\succ_{I}, A V\right)$. Moreover, recall that we can restrict attention to sincere strategies in determining $S\left(\succ_{I}, V\right)$ (Lemma 2).

\footnotetext{
28 For example, a rule that yields $S(\cdot, V)=A$ at every preference profile would trivially satisfy MON, but hardly make for an appealing voting procedure.

29 Gärdenfors (1976), even as he extends the analysis of Gibbard (1973) and Satterthwaite (1975) to nonresolute social choice correspondences, warns that those correspondences that are insufficiently decisive may be of little practical interest.
} 
Case (i): Condition (1) is violated, i.e., there are no $x, y \in A$ such that $M(x)>$ $M(y)+S M(y)$. Then $S\left(\succ_{I}, A V\right)=A$. To see that $S\left(\succ_{I}, V\right)=A$, let $M(x) \geq$ $M(y), M(z)$ and consider a ballot profile where scores of either 1 or 0 are assigned as follows: (i) $x$ is approved of by all $M(x)$ voters in $I_{x y z} \cup I_{x z y}$, (ii) $y$ is approved of by all $M(y)$ voters in $I_{y x z} \cup I_{y z x}$ and by $M(x)-M(y) \leq S M(y)$ voters in $I_{x y z} \cup I_{z y x}$, and (iii) $z$ is approved of by all $M(z)$ voters in $I_{z x y} \cup I_{z y x}$ and by $M(x)-M(z) \leq S M(z)$ voters in $I_{x z y} \cup I_{y z x}$. This yields a three-way tie with $v^{x}=v^{y}=v^{z}=M(x)$. For voters approving of their most-preferred candidate only, any deviation would rule out its election, so would be worse for some tiebreaker $\triangleright$. For voters approving of their two most-preferred candidates, any deviation would rule out the election of one of them while preserving the least preferred as possible outcome-a worse reply for some $\triangleright$. Hence the ballot profile just constructed survives under iterative elimination.

Case (ii): Condition (1), but not (2), is satisfied, i.e., we can label candidates such that $M(x)>M(z)+S M(z)$ but no majority prefers $x$ or $y$ over the other. Then $S\left(\succ_{I}, A V\right)=\{x, y\}$. To see that $\{x, y\} \subseteq S\left(\succ_{I}, V\right)$, consider ballots that assign a score of 1 to a voter's preferred candidate among $x$ and $y$ and a score of 0 to the other. For any $V_{i}^{n}$, denote the subset of such ballots as $\hat{V}_{i}^{n}$. Since $V$ is an EAV-rule, $\hat{V}_{i}^{1}$ is non-empty. For the induction step, take $\Gamma\left(\succ_{I}, V^{n}\right)$; at any ballot profile $v \in \hat{V}^{n}$, we have $v^{x}=v^{y} \geq M(x)>M(z)+S M(z) \geq v^{z}$. Thus, deviating and assigning a lower score to the preferred candidate among $x$ and $y$, or a higher score to the other, would be a worse reply for each voter. Hence, no strategy outside of $\hat{V}_{i}^{n}$ dominates any strategy within. But then some strategy within $\hat{V}_{i}^{n}$ survives elimination of dominated strategies and is included in $V_{i}^{n+1}$. We thus find, by induction, that a tie of $x$ and $y$ survives under iterative elimination of dominated strategies.

Case (iii)(a): Condition (1) and (2) are satisfied, in that we can label candidates so that $M(x)>M(z)+S M(z)$ and a majority prefers $x$ over $y$. Then $S\left(\succ_{I}, A V\right)=\{x\}$. We want to show that $x \in S\left(\succ_{I}, V\right)$, i.e., survives as a possible outcome in $\Gamma\left(\succ_{I}, V^{\bar{m}}\right)$.

Suppose $v_{i}=\left(v_{i}^{x}, v_{i}^{y}, v_{i}^{z}\right)=(1,0,0)$ survives and is included in $V_{x y z}^{k}$ for all $k \leq \bar{m}$. Then at any profile $v \in V^{k}$ where all $i \in I_{x y z}$ choose $v_{i}=(1,0,0)$, we have $v^{x}>v^{z}$ (since $M(x)>M(z)+S M(z)$ is equivalent to $\left|I_{x y z}\right|>\left|I_{z x y}\right|+\left|I_{z y x}\right|+\left|I_{y z x}\right|$ and since by Lemma 2 we can assume w.l.o.g. that voters in $I_{x z y} \cup I_{y x z}$ award a weakly higher score to $x$ than to $z)$. For $k=1$, assuming that all $i \in I_{x y z}$ choose $v_{i}=(1,0,0)$ and rule out $z$, any voter $j \in I_{x z y}$ finds $v_{j}=(1,0,0)$ to be a best reply, given that it maximizes their impact on $v^{x}-v^{y}$. Likewise, any voter $j \in I_{z x y}$ finds $v_{j}^{\prime}=(1,0,1)$ to be a best reply. If all choose $j \in I_{x z y} \cup I_{z x y}$ choose $v_{j}$, resp. $v_{j}^{\prime}$, then $v^{x}>v^{y}$ given that $x$ is majority preferred over $y$. Hence, $x$ wins the election.

Moreover, any strategy dominating $v_{j}$ or $v_{j}^{\prime}$ (possibly at some $\Gamma\left(\succ_{I}, V^{k}\right.$ ) with $k>1$ ) has likewise to be a best reply against any profile $v \in V^{k} \subset V^{1}$ where $v_{i}=(1,0,0)$ for all $i \in I_{x y z}$, so lead to the same outcome. Hence, even if $v_{j}$ or $v_{j}^{\prime}$ are eventually eliminated, the surviving strategies dominating them preserve $x$ as an outcome when used by all $j \in I_{x z y} \cup I_{z x y}$ while all $i \in I_{x y z}$ use $v_{i}=(1,0,0)$.

Finally, if $v_{i}=(1,0,0)$ is dominated by $v_{i}^{*}$ for $i \in I_{x y z}$ in $\Gamma\left(\succ_{I}, V^{k}\right), k \geq 1$, then $v_{i}^{*}$ must likewise rule out $z$ as an outcome in $\Gamma\left(\succ_{I}, V^{k}\right)$ if all $i \in I_{x y z}$ choose it. Moreover, at any profile $v \in V^{k}$, once $z$ is ruled out, $v_{i}$ is a best response for 
$I_{x y z}$ as maximizes the score difference $v^{x}-v^{y}$. Thus, for any opposing strategy profile $v_{-x y z}=\left(v_{j}\right)_{j \in I \backslash I_{x y z}}$ whether all $i \in I_{x y z}$ choose $v_{i}^{*}$ or whether all choose $v_{i}=(1,0,0)$ yields the same outcome. Hence, even if $v_{i}$ is eliminated, the strategy $v_{i}^{*}$ dominating it allows for the same argument involving voters $I_{x z y} \cup I_{z x y}$ above and so preserves $x$ as an outcome in all $\Gamma\left(\succ_{I}, V^{k^{\prime}}\right), k^{\prime} \geq k$, as long as $v_{i}^{*}$ is present. Induction over the sequence of strategies that iteratively dominate $v_{i}=(1,0,0)$ completes the argument.

Case (iii)(b): Condition (1) and (2) are satisfied, in that we can label candidates so that $M(y)>M(z)+S M(z)$ and a majority prefers $x$ over $y$. Then $S\left(\succ_{I}, A V\right)=\{x\}$. We want to show that $x \in S\left(\succ_{I}, V\right)$, i.e., survives as a possible outcome in $\Gamma\left(\succ_{I}, V^{\bar{m}}\right)$.

Analogous to (iii)(a), as long as $v_{i}=\left(v_{i}^{x}, v_{i}^{y}, v_{i}^{z}\right)=(0,1,0)$ is included in $V_{y x z}^{k}$, any profile where all $i \in I_{y x z}$ choose $v_{i}$ rules out $z$, which makes $(1,0,0)$ a best reply for all $j \in I_{x y z} \cup I_{x z y}$ and $(1,0,1)$ a best reply for $j \in I_{z x y}$, at least as long as these strategies have not been eliminated. If these strategies are used, $x$ wins as voters $I_{x y z} \cup I_{x z y} \cup I_{z x y}$ constitute the majority. Even if these strategies should eventually be eliminated, the strategies dominating them have to likewise be a best response in the restricted game and hence yield outcome $x$. Finally, again analogously to (iii)(a), if $v_{i}=(0,1,0)$ is dominated by $v_{i}^{*}$ in some restricted game, a profile where all $i \in I_{y x z}$ use $v_{i}^{*}$ instead of $v_{i}$ has to yield the same outcome in the restricted game-and hence allows for the same argument concerning voters in $I_{x y z} \cup I_{x z y} \cup I_{z x y}$. Induction over strategies iteratively dominating $v_{i}$ concludes.

One might think that adding to the set of admissible ballots, as we do when we move from AV to any other EAV-rule, would in general lead to a larger solution as there are potentially more strategies that need to be ruled out to narrow down the set of possible outcomes. However this simple intuition is wrong-for example, AV is itself an extension of the Plurality rule and yet satisfies $\mathbf{U}$ while the latter may fail to narrow down the solution to a unanimously preferred candidate.

Last, observe that Cumulative Voting suffers from the same defect as Plurality rulevoters who all agree on their most preferred candidate $a$ but are split on the ranking of $b$ and $c$, may assign their whole budget in support of the 'lesser evil' which yields a tie between $b$ and $c$ and hence a violation of $\mathbf{U}$.

\subsection{Binary voting trees}

By focussing our analysis on scoring rules, we have in particular restricted our attention to voting rules that are neutral with respect to candidates. ${ }^{30}$ If we are willing to give up neutrality, majority-voting along a binary tree (with pre-determined tie-breaks at each node) offers a well known class of procedures that satisfy CON, i.e., ensure that a (strict) Condorcet-winner will be elected when voters use iteratively undominated strategies.

However they may exhibit violations of monotonicity. Moulin (1986) documents such a violation in a setup with 4 candidates for trees where multiple terminal nodes

\footnotetext{
30 Myerson (1995) shows that these are the only (candidate-)neutral and (voter-)anonymous rules that satisfy reinforcement and overwhelming majority (for joined and replicated ballot profiles).
} 

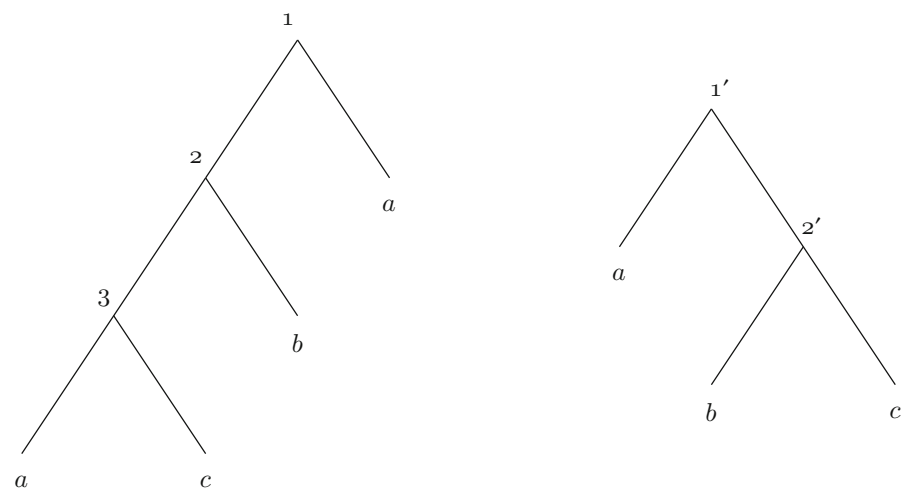

Fig. 3 Two binary voting tree. Ties broken to the right

arrive at the same voting outcome. In his example there are no broken ties, but if we allow for (pre-determined) tie-breaking then monotonicity ${ }^{31}$ may already be violated for three candidate, as the following minimal example demonstrates.

Consider the voting tree on the left hand side of Fig. 3 and suppose $I=I_{a c b} \cup I_{b a c} \cup$ $I_{b c a} \cup I_{c b a}$ with $\left|I_{a c b}\right|=\left|I_{c b a}\right|=2$ and $\left|I_{b a c}\right|=\left|I_{b c a}\right|=1$. Then $a$ is the unique solution in iteratively undominated strategies: eliminating dominated strategies lets voters vote sincerely at node 3 which gives a tie of $a$ and $c$, broken in favour of $c$. In turn, this makes it dominant for the majority $I_{a c b} \cup I_{c b a}$ to vote against $b$ at node 2 . But then the choice at node 1 is between $c$ and $a$ for which the use of undominated strategies yields a tie between $a$ and $c$, broken in favour of $a$.

However if $a$ 's popularity increases slightly as one voter moves from $I_{b c a}$ to $I_{b a c}$, so that we now have a Condorcet cycle in which $a$ is majority-preferred over $c$, then $a$ would be elected at node 3 . This makes it dominant for the majority $I_{b a c} \cup I_{c b a}$ to vote for $b$ at node 2 . The same majority would then also vote against $a$ at node 1 so that $b$ is the winner after $a$ 's increase in popularity.

Nonetheless, there are binary voting trees that not only satisfy CON but also ensure monotonicity. Consider the tree on the right hand side of Fig. 3.

For $c$ to be the outcome at some profile $\succ_{I}$, it must enjoy weak majority support over both $b$ and $a$-but then this is still the case at any $c$-monotonic transformation of $\succ_{I}$ and $c$ remains the election outcome after gaining popularity.

For $b$ to be the outcome at some profile $\succ_{I}$, more than half of all voters need to prefer it over $c$ and at least half the voters over $a$. Again, it will then remain the election outcome at any $b$-monotonic transformation of $\succ_{I}$.

At all remaining profiles $\succ_{I}, a$ is the election outcome. Since an $a$-monotonic transformation cannot lead $b$ or $c$ to gain majority support (weak or strong) over $a$ and since it also does not affect voter's preferences over the pair $b$ and $c$, the outcome will not change, neither to $b$ nor to $c-a$ remains the election outcome.

\footnotetext{
31 For pre-determined tie-braking the solution is single-valued at every profile and our monotonicity axioms collapse into one.
} 


\section{Summary and concluding remarks}

Within the class of scoring rules we were are able to characterize voting procedures using a small number of intuitive axioms that are based on the idea of majoritarianism, extending it to situations with three candidates, and monotonicity. In particular, Approval Voting and the Borda Rule stand out as optimal voting procedures with respect to our axioms.

For the subset of direct mechanism scoring rules, i.e., rules that are at most as complex as classical positional scoring rules, the Borda Rule is the only rule that both ensures a minimal degree of majoritarianism (as embodied by unanimity, $\mathbf{U}$, and, in the presence of a unanimously agreed worst alternative, MEW) as well as monotonicity (MON).

Within the larger class of all finite scoring rules, we identify extensions of Approval Voting as precisely the class of rules that satisfies a slightly stronger notion of majoritarianism, MEPD (i.e., elect the majority preferred among, at most, two Pareto-efficient alternatives), and show that Approval Voting is not only the simplest rule within that class, but also the most decisive. Moreover, while Approval Voting satisfies weak monotonicity, no scoring rule that likewise satisfies MEPD will satisfy the stronger monotonicity notion MON*. In addition, we show that strengthening our majoritarianism notion further by requiring Condorcet-consistency leads to an impossibility - no finite scoring rule satisfies CON.

The analysis raises a number of open questions. Are there scoring rules that satisfy strong monotonicity (sMON) or at least MON* together with arguably the most basic notions of majoritarianism in the presence of three canddiate, $\mathbf{U}$ and MEW? For direct mechanisms, Corollary 4 provides a negative answer-but what about general (finite) scoring rules? Is it possibles for a scoring rule to simultaneously satisfy MEPD and MON? Do there exists anonymous and neutral rules that satisfy CON, or are we forced to forego neutrality as when voting on a binary tree?

For elections involving more than three candidates, one may ask whether our axioms, MEPD or MEW, can be extended so as to yield analogous characterisations of Approval Voting and the Borda Rule. For example, one might extent MEPD to the case of $n \geq 4$ alternatives by requiring that whenever there are only two Pareto-efficient alternatives, the majority-preferred among the two should be elected. However, even Approval Voting would violate this generalisation of MEPD. ${ }^{32}$ Do there exist scoring rules or, more generally, anonymous and neutral rules that satisfies it?

We hope that questions such as these will stimulate future research.

$\overline{32 \text { Example available upon request. }}$ 
Acknowledgements I thank Tilman Börgers, Micael Castanheira, Sébastien Courtin, Frank Heinemann and Matías Núñez as well as participants at the 9th Conference on Economic Design in Istanbul and the 26th International Conference on Game Theory in Stony Brook for their helpful comments and suggestions.

Funding Open Access funding enabled and organized by Projekt DEAL. Support by German Science Foundation through CRC TRR 190 and Fonds de la Recherche Scientifique-FNRS, fellowship-grant $\mathrm{n}^{\circ} 1 . \mathrm{B} \cdot 222.17 \mathrm{~F}$, is gratefully acknowledged.

Open Access This article is licensed under a Creative Commons Attribution 4.0 International License, which permits use, sharing, adaptation, distribution and reproduction in any medium or format, as long as you give appropriate credit to the original author(s) and the source, provide a link to the Creative Commons licence, and indicate if changes were made. The images or other third party material in this article are included in the article's Creative Commons licence, unless indicated otherwise in a credit line to the material. If material is not included in the article's Creative Commons licence and your intended use is not permitted by statutory regulation or exceeds the permitted use, you will need to obtain permission directly from the copyright holder. To view a copy of this licence, visit http://creativecommons.org/licenses/by/4.0/.

\section{Appendix}

Example 4 Consider two voters, $i$ and $j$ with preferences $a \succ_{i} b \succ_{i} c$ and $c \succ_{i} a \succ_{i} b$, who elect a candidate via Borda Rule voting. Ties are broken uniformly at random and $i$ is indifferent between $b$, a tie of $a$ and $c$, as well as a three-way tie. Similarly, $j$ is indifferent between $a$, a tie of $b$ and $c$, and a three-way tie. After eliminating dominated strategies (where one assigns a higher score to the least- than to the most-preferred candidate), the reduced game has the following representation.

\begin{tabular}{llll}
\hline$\left(v_{j}^{a}, v_{j}^{b}, v_{j}^{c}\right)$ & $\left(v_{i}^{a}, v_{i}^{b}, v_{i}^{c}\right)$ & & \\
\cline { 2 - 4 } & $\left(1, \frac{1}{2}, 0\right)$ & $\left(1,0, \frac{1}{2}\right)$ & $\left(\frac{1}{2}, 1,0\right)$ \\
\hline$\left(1,0, \frac{1}{2}\right)$ & $a$ & $a$ & $a$ \\
$\left(\frac{1}{2}, 0,1\right)$ & $a$ & tie: $a, c$ & tie: $a, b, c$ \\
$\left(0, \frac{1}{2}, 1\right)$ & tie: $a, b, c$ & $c$ & $b$ \\
\hline
\end{tabular}

Note that for $i, v_{i}=\left(1, \frac{1}{2}, 0\right)$ weakly dominates the other two remaining strategies. If we first eliminate $\left(1,0, \frac{1}{2}\right)$, then for voter $j$ we find that $v_{j}=\left(0, \frac{1}{2}, 1\right)$ is dominated (by $\left.\left(1,0, \frac{1}{2}\right)\right)$. Eliminating $v_{j}$ and finally also $\left(\frac{1}{2}, 1,0\right)$ for voter $i$, we arrive at $a$ as the only remaining outcome.

If instead we begin by eliminating voter $i$ 's strategy $\left(\frac{1}{2}, 1,0\right)$, then for voter $j$ we have $v_{j}=\left(0, \frac{1}{2}, 1\right)$ as a strategy that dominates the other two. Eliminating both before finally eliminating $\left(1,0, \frac{1}{2}\right)$ as voter $i$ 's strategy, we are left with a three-way tie as the only remaining outcome-which is strictly worse from $i$ 's perspective.

Proof of Lemma 1 Consider a ballot $w=(k, l, m) \in V$ and assume w.1.o.g. that $k \geq l \geq m$. Since $V$ is assumed to be neutral, it also includes all permutations of $w$.

If $k>l>m$, the 6 permutations exhaust $V$; normalizing all ballots in $V$, replacing $k$ by $k^{\prime}=\frac{k-m}{k-m}=1, l$ by $l^{\prime}=\frac{l-m}{k-m} \in(0,1)$ and $m$ by $m^{\prime}=\frac{m-m}{k-m}=0$, yields case (1). 
If two entries of $w$ coincide, $V$ contains 3 permutations of $w$. If those are the only elements of $V$, normalizing ballots as before again yields case (1). If $V$ contains another non-abstention ballot $w^{\prime}=(p, q, r)$, then two of its three entries must coincide - otherwise the permutations of $w$ and $w^{\prime}$ would exceed $|V| \leq 6$.

W.l.o.g assume $p \geq q \geq r$. If $k=l>m$ and $p>q=r$, normalizing each permutation of $w$ by subtracting $m$ and each permutation of $w^{\prime}$ by subtracting $r$ before dividing all scores by $k-m+p-r$ yields $k^{\prime}=l^{\prime}=\frac{k-m}{k-m+p-r}, m^{\prime}=0, p^{\prime}=\frac{p-r}{k-m+p-r}$ and $q^{\prime}=r^{\prime}=0$, which corresponds to case (2).

If $k>l=m$ and $p>q=r$, assume w.l.o.g. that $k-m \geq p-r$. Normalizing each permutation of $w$ by subtracting $m$ and each permutation of $w^{\prime}$ by subtracting $r$ before dividing all scores by $k-m$ yields $k^{\prime}=1, l^{\prime}=m^{\prime}=0, p^{\prime}=\frac{p-r}{k-m} \leq 1$ and $q^{\prime}=r^{\prime}=0$, which corresponds to case (3).

If $k=l>m$ and $p=q>r$, assume w.l.o.g. that $k-m \geq p-r$. Normalizing each permutation of $w$ by subtracting $m$ and each permutation of $w^{\prime}$ by subtracting $r$ before dividing all scores by $k-m$ yields $k^{\prime}=l^{\prime}=1, m^{\prime}=0, p^{\prime}=q^{\prime}=\frac{p-r}{k-m} \leq 1$ and $r^{\prime}=0$, which corresponds to case (4).

Proof of Theorem 1 First, consider the if part for the remaining cases (2) and (3). In particular, assume (2) holds for some labelling of candidates, so that w.l.o.g.

$$
\begin{aligned}
\min _{v \in V^{1}} v^{a}-v^{c}= & 1 / 2\left|I_{a b c}\right|-1 / 2\left|I_{a c b}\right| \\
& -1 / 2\left|I_{b a c}\right|-\left|I_{b c a}\right|-\left|I_{c a b}\right|-\left|I_{c b a}\right| \geq 0 \text { and }\left|I_{c a b}\right|>\left|I_{b a c}\right| .
\end{aligned}
$$

Then, for any $i \in I_{a b c}$ in $\Gamma\left(\succ_{I}, V^{1}\right)$, ballot $v_{i}=\left(v_{i}^{a}, v_{i}^{b}, v_{i}^{c}\right)=(1,1 / 2,0)$ is a weakly better reply than $\tilde{v}_{i}=(1 / 2,1,0)$ against any $v_{-i} \in V_{-i}^{1}$ :

(i) if for $\tilde{v}=\left(\tilde{v}_{i}, v_{-i}\right), \tilde{v}^{b}>\tilde{v}^{a} \geq \tilde{v}^{c}$, then for $v=\left(v_{i}, v_{-i}\right)$, we have $v^{a}>v^{c}$,

(ii) if for $\tilde{v}=\left(\tilde{v}_{i}, v_{-i}\right), \tilde{v}^{a} \geq \tilde{v}^{b}, \tilde{v}^{c}$, then for $v=\left(v_{i}, v_{-i}\right)$, we have $v^{a}>v^{b}, v^{c}$.

Hence, $\tilde{v}_{i}$ is either dominated by $v_{i}=(1,1 / 2,0)$, or is redundant to it. Eliminating $\tilde{v}_{i}$ and moving to the restricted game, $\Gamma\left(\succ_{I}, V^{\prime}\right)$, where $V_{a b c}^{\prime}=V_{a b c}^{1} \backslash\{(1 / 2,1,0)\}=$ $\{(1,0,1 / 2),(1,1 / 2,0)\}$ and $V_{j}^{\prime}=V_{j}^{1}$ for all $j \notin I_{a b c}$ we find that

$$
\begin{gathered}
\min _{v \in V^{\prime}} v^{a}-v^{b}=1 / 2\left|I_{a b c}\right|+1 / 2\left|I_{a c b}\right|-\left|I_{b a c}\right|-\left|I_{b c a}\right|-1 / 2\left|I_{c a b}\right|-\left|I_{c b a}\right| \\
=\underbrace{1 / 2\left|I_{a b c}\right|-1 / 2\left|I_{a c b}\right|-1 / 2\left|I_{b a c}\right|-\left|I_{b c a}\right|-\left|I_{c a b}\right|-\left|I_{c b a}\right|}_{\geq 0} \\
\underbrace{-1 / 2\left|I_{b a c}\right|+1 / 2\left|I_{c a b}\right|}_{>0}+\left|I_{a c b}\right|>0,
\end{gathered}
$$

which rules out $b$ as an outcome of $\Gamma\left(\succ_{I}, V^{\prime}\right)$. But then, in the game $\Gamma\left(\succ_{I}, V^{\prime}\right)$, for any voter $i$ who prefers $a$ over $c, v_{i}=(1,1 / 2,0)$ is a best reply as it maximizes $i$ 's impact on $v^{a}-v^{c}$. Eliminating dominated or redundant strategies and moving to $\Gamma\left(\succ_{I}, V^{\prime \prime}\right)$, where $V_{i}^{\prime \prime}=\{(1,1 / 2,0)\}$ for all $i \in I_{a b c} \cup I_{a c b} \cup I_{b a c}$ and $V_{j}^{\prime \prime}=V_{j}^{\prime}=V_{j}^{1}$ 
for all $j \in I_{b c a} \cup I_{c a b} \cup I_{c b a}$, we find that for all $v \in V^{\prime \prime}$

$$
\begin{aligned}
v^{a} \geq\left|I_{a b c}\right|+\left|I_{a c b}\right|+\left|I_{b a c}\right| & >2\left|I_{a c b}\right|+2\left|I_{b a c}\right|+2\left|I_{b c a}\right|+2\left|I_{c a b}\right|+2\left|I_{c b a}\right|-1 \\
& \geq\left|I_{b c a}\right|+\left|I_{c a b}\right|+\left|I_{c b a}\right| \geq v^{c}
\end{aligned}
$$

where the strict inequality follows from directly from condition (2), while the next weak inequality follows from the fact that $\left|I_{c a b}\right|>\left|I_{b a c}\right| \geq 0$. Hence, $a$ is the unique outcome after iteratively eliminating dominated strategies from $\Gamma\left(\succ_{I}, V^{0}\right)$.

Finally, assume condition (3) holds for some labelling of candidates, so that w.l.o.g.

$$
\begin{aligned}
\min _{v \in V^{1}} v^{a}-v^{c}= & 1 / 2\left|I_{a b c}\right|-1 / 2\left|I_{a c b}\right|-1 / 2\left|I_{b a c}\right|-\left|I_{b c a}\right| \\
& -\left|I_{c a b}\right|-\left|I_{c b a}\right| \geq-1 / 2 \text { and }\left|I_{a c b}\right|>0 .
\end{aligned}
$$

Then, for any $i \in I_{a c b}$ in $\Gamma\left(\succ_{I}, V^{1}\right)$, ballot $v_{i}=\left(v_{i}^{a}, v_{i}^{b}, v_{i}^{c}\right)=(1,0,1 / 2)$ is a weakly better reply than $\tilde{v}_{i}=(1 / 2,0,1)$ against any $v_{-i} \in V_{-i}^{1}$ :

(i) if for $\tilde{v}=\left(\tilde{v}_{i}, v_{-i}\right), \tilde{v}^{a} \geq \tilde{v}^{b}$, then for $v=\left(v_{i}, v_{-i}\right)$, we have $v^{a}>v^{b}, v^{c}$,

(ii) if for $\tilde{v}=\left(\tilde{v}_{i}, v_{-i}\right), \tilde{v}^{b}>\tilde{v}^{a}, \tilde{v}^{c}$, then $v_{i}$ can only improve the outcome,

(iii) if for $\tilde{v}=\left(\tilde{v}_{i}, v_{-i}\right), \tilde{v}^{c} \geq \tilde{v}^{b}>\tilde{v}^{a}$, then $\tilde{v}^{c}=\tilde{v}^{a}+1 / 2$. Hence $\tilde{v}^{b}=\tilde{v}^{c}$ and $2\left(\tilde{v}^{a}+\tilde{v}^{b}+\tilde{v}^{c}\right)=6 \tilde{v}^{a}+2$. However, as each voter awards scores that sum to $\frac{3}{2}, 2\left(\tilde{v}^{a}+\tilde{v}^{b}+\tilde{v}^{c}\right)$ would have to be divisible by three - a contradiction.

Hence, $\tilde{v}_{i}$ is either dominated by $v_{i}=(1,0,1 / 2)$, or is redundant to it. Eliminating $\tilde{v}_{i}$ and moving to the restricted game, $\Gamma\left(\succ_{I}, V^{\prime}\right)$, where $V_{a c b}^{\prime}=V_{a c b}^{1} \backslash\left\{\left(\frac{1}{2}, 0,1\right)\right\}=$ $\left\{\left(1,0, \frac{1}{2}\right),\left(1, \frac{1}{2}, 0\right)\right\}$ and $V_{j}^{\prime}=V_{j}^{1}$ for all $j \notin I_{a c b}$, condition (3) yields

$$
\begin{aligned}
& \min _{v \in V^{\prime}} v^{a}-v^{c} \\
& =1 / 2\left|I_{a b c}\right|+1 / 2\left|I_{a c b}\right|-1 / 2\left|I_{b a c}\right|-\left|I_{b c a}\right|-\left|I_{c a b}\right|-\left|I_{c b a}\right| \\
& =\underbrace{1 / 2\left|I_{a b c}\right|-1 / 2\left|I_{a c b}\right|-1 / 2\left|I_{b a c}\right|-\left|I_{b c a}\right|-\left|I_{c a b}\right|-\left|I_{c b a}\right|+1}_{>0} \underbrace{1+1+\left|I_{a c b}\right|}_{\geq 0}>0,
\end{aligned}
$$

which rules out $c$ as an outcome of $\Gamma\left(\succ_{I}, V^{\prime}\right)$. But then, in the game $\Gamma\left(\succ_{I}, V^{\prime}\right)$, for any voter $i$ who prefers $a$ over $b, v_{i}=(1,0,1 / 2)$ is a best reply as it maximizes $i$ 's impact on $v^{a}-v^{b}$. Eliminating strategies dominated by (or redundant to) $v_{i}$ for these voters, we arrive at $\Gamma\left(\succ_{I}, V^{\prime \prime}\right)$, where $V_{i}^{\prime \prime}=\{(1,0,1 / 2)\}$ for all $i \in I_{a b c} \cup I_{a c b} \cup I_{c a b}$ and $V_{i}^{\prime \prime}=V_{i}^{\prime}=V_{i}^{1}$ for all $i \in I_{b a c} \cup I_{b c a} \cup I_{c b a}$, so that for all $v \in V^{\prime \prime}$

$$
\begin{aligned}
v^{a} \geq\left|I_{a b c}\right|+\left|I_{a c b}\right|+\left|I_{c a b}\right| & >\underbrace{2\left|I_{a c b}\right|}_{\geq 2}+\left|I_{b a c}\right|+2\left|I_{b c a}\right|+3\left|I_{c a b}\right|+2\left|I_{c b a}\right|-2 \\
& \geq\left|I_{b a c}\right|+\left|I_{b c a}\right|+\left|I_{c b a}\right| \geq v^{b}
\end{aligned}
$$

by condition (3). Hence, $a$ is the unique outcome after iteratively eliminating dominated strategies from $\Gamma\left(\succ_{I}, V^{0}\right)$. This ends the proof of the if part of Theorem 1 . 
Next, consider the only if part. We will show that as conditions (1)-(3) are violated, each election outcome is possible under some ballot profile, where each voter chooses a strategy that is iteratively undominated. For that we make use of the following facts.

Claim 1 Consider a ballot profile $v$ such that $v^{x}=v^{y}=v^{z}$ and some voter $i \in I_{x y z}$ such that $v_{i}^{x}=1$. Then $v_{i}$ is undominated in any game $\Gamma\left(\succ_{I}, V^{\prime}\right)$ where $v \in V^{\prime} \subseteq V^{1}$.

Proof. Consider the case $v_{i}=\left(v_{i}^{x}, v_{i}^{y}, v_{i}^{z}\right)=(1,1 / 2,0)$. If $x \triangleright y, z$, then $i$ 's most preferred outcome $x$ is realized. In contrast, a switch to $\tilde{v}_{i}=(1 / 2,1,0)$ would yield outcome $y$, a switch to $\tilde{v}_{i}=(1,0,1 / 2)$ would yield $z$. Hence $v_{i}=(1,1 / 2,0)$ is undominated.

If $v_{i}=\left(v_{i}^{x}, v_{i}^{y}, v_{i}^{z}\right)=(1,0,1 / 2)$ and $x \triangleright y, z$ outcome $x$ is realized, while a switch to $\tilde{v}_{i}=(1 / 2,1,0)$ or $\tilde{v}_{i}=(1,1 / 2,0)$ would yield $y$. Hence $v_{i}=(1,0,1 / 2)$ is undominated.

Claim $1 \diamond$

Claim 2 Consider a ballot profile $v$ such that $v^{x}=1 / 2|I|-1 / 2, v^{y}=1 / 2|I|+1 / 2$, and $v^{z}=1 / 2|I|$ and some voter $i \in I_{x y z}$ such that $v_{i}=\left(v_{i}^{x}, v_{i}^{y}, v_{i}^{z}\right)=(1 / 2,1,0)$. Then $v_{i}$ is undominated in any game $\Gamma\left(\succ_{I}, V^{\prime}\right)$ where $v \in V^{\prime} \subseteq V^{1}$.

Proof. Profile $v$ leads to the election of $i$ 's second most preferred alternative, independent of $\triangleright$. If $i$ switches to any other undominated strategy, i.e., $(1,1 / 2,0),(1,0,1 / 2) \in$ $V_{i}^{1}$, then her least preferred alternative $z$ would be elected for $z \triangleright x, y$. Hence, $v_{i}=(1 / 2,1,0)$ is undominated in any game $\Gamma\left(\succ_{I}, V^{\prime}\right)$ where $v \in V^{\prime} \subseteq V^{1}$. Claim $2 \diamond$

To fix notation and without loss of generality, let us assume that $\left|I_{a b c}\right| \geq\left|I_{a c b}\right|$ and $\left|I_{a b c}\right|+\left|I_{a c b}\right| \geq\left|I_{b a c}\right|+\left|I_{b c a}\right| \geq\left|I_{c a b}\right|+\left|I_{c b a}\right|$. Since, by assumption, (1) is violated, we have

$$
\left|I_{a b c}\right| \leq\left|I_{a c b}\right|+\left|I_{b a c}\right|+2\left|I_{b c a}\right|+2\left|I_{c a b}\right|+2\left|I_{c b a}\right| \text {. }
$$

Case 1: $\left|I_{a b c}\right|=\left|I_{a c b}\right|+\left|I_{b a c}\right|+2\left|I_{b c a}\right|+2\left|I_{c a b}\right|+2\left|I_{c b a}\right|$

Since by assumption conditions (2) and (3) are violated, we have $\left|I_{c a b}\right| \leq\left|I_{b a c}\right|$ and $\left|I_{a c b}\right|=0$. If $\left|I_{b c a}\right|+\left|I_{c a b}\right|+\left|I_{c b a}\right|=0$, so that $\left|I_{a b c}\right|=\left|I_{b a c}\right|$, consider a ballot profile $v$ where all $i \in I_{a b c}$ chose $(1,0,1 / 2) \in V_{a b c}^{1}$ while all $i \in I_{b a c}$ chose $(0,1,1 / 2) \in V_{b a c}^{1}$. Then $v^{a}=v^{b}=v^{c}$, by Claim 1 both strategies are undominated in any game $\Gamma\left(\succ_{I}, V^{\prime}\right)$ where $v \in V^{\prime} \subseteq V^{1}$, and hence no outcome can be ruled out via iterated elimination of dominated strategies.

Next, consider $\left|I_{b c a}\right|+\left|I_{c a b}\right|+\left|I_{c b a}\right|>0$ and construct two strategy profiles as follows:

Profile $v \in V^{1}$ :

- Let all $i \in I_{a b c}$ choose $v_{i}=(1,0,1 / 2)$,

- Let all $i \in I_{b a c}$ choose $v_{i}=(0,1,1 / 2)$,

- Let all $i \in I_{b c a} \cup I_{c a b} \cup I_{c b a}$ choose $v_{i}=(0,1 / 2,1)$. 
Then,

$$
v^{a}-v^{c}=1 / 2\left|I_{a b c}\right| \underbrace{-1 / 2\left|I_{b a c}\right|-\left|I_{b c a}\right|-\left|I_{c a b}\right|-\left|I_{c b a}\right|}_{-1 / 2\left|I_{a b c}\right|}=0,
$$

while

$$
\begin{aligned}
v^{a}-v^{b} & =\left|I_{a b c}\right|-\left|I_{b a c}\right|-1 / 2\left|I_{b c a}\right|-1 / 2\left|I_{c a b}\right|-1 / 2\left|I_{c b a}\right| \\
& =3 / 2\left|I_{b c a}\right|+3 / 2\left|I_{c a b}\right|+3 / 2\left|I_{c b a}\right| \geq 3 / 2,
\end{aligned}
$$

so that both $a$ and $c$ are possible outcomes, depending on $\triangleright$. If $c \triangleright a$, then $c$ is elected while any unilateral deviation to some $\tilde{v}_{i} \in V_{i}^{1}$ by some $i \in I_{b c a} \cup I_{c a b} \cup I_{c b a}$ would yield outcome $a$. Hence, for $i \in I_{b c a} \cup I_{c a b} \cup I_{c b a},(0,1 / 2,1)$ is the unique best response and thus undominated in any game $\Gamma\left(\succ_{I}, V^{\prime}\right)$ where $v \in V^{\prime} \subseteq V^{1}$.

Profile $\hat{v} \in V^{1}$ :

- Let $\left|I_{b a c}\right|-\left|I_{c a b}\right|$ of $I_{a b c}$ chose $\hat{v}_{i}=(1,0,1 / 2)$

- Let the remaining $2\left|I_{b c a}\right|+3\left|I_{c a b}\right|+2\left|I_{c b a}\right|>0$ voters of $I_{a b c}$ choose $\hat{v}_{i}=$ $(1,1 / 2,0)$

- Let all $i \in I_{c a b}$ choose $\hat{v}_{i}=(0,1 / 2,1)$,

- Let all $i \in I_{b a c} \cup I_{b c a} \cup I_{c b a}$ chooses $\hat{v}_{i}=(0,1,1 / 2)$.

Then,

$$
\begin{aligned}
\left|\hat{v}^{a}\right|-\left|\hat{v}^{b}\right| & =1 / 2\left|I_{a b c}\right|+1 / 2\left(\left|I_{b a c}\right|-\left|I_{c a b}\right|\right)-1 / 2\left|I_{c a b}\right|-\left|I_{b a c}\right|-\left|I_{b c a}\right|-\left|I_{c b a}\right| \\
& =1 / 2\left|I_{a b c}\right| \underbrace{-1 / 2\left|I_{b a c}\right|-\left|I_{b c a}\right|-\left|I_{c a b}\right|-\left|I_{c b a}\right|}_{=1 / 2\left|I_{a b c}\right|}=0,
\end{aligned}
$$

while

$$
\begin{aligned}
\left|\hat{v}^{a}\right|-\left|\hat{v}^{c}\right| & =\left|I_{a b c}\right|-1 / 2\left(\left|I_{b a c}\right|-\left|I_{c a b}\right|\right)-\left|I_{c a b}\right|-1 / 2\left|I_{b a c}\right|-1 / 2\left|I_{b c a}\right|-1 / 2\left|I_{c b a}\right| \\
& =\left|I_{a b c}\right|-\left|I_{b a c}\right|-1 / 2\left|I_{b c a}\right|-1 / 2\left|I_{c a b}\right|-1 / 2\left|I_{c b a}\right| \\
& =3 / 2\left|I_{b c a}\right|+3 / 2\left|I_{c a b}\right|+3 / 2\left|I_{c b a}\right| \geq 3 / 2,
\end{aligned}
$$

so that both $a$ and $b$ are possible outcomes, depending on $\triangleright$. If $b \triangleright a$, then $b$ is elected while any unilateral deviation to some $\tilde{v}_{i} \in V_{i}^{1}$ by some $i \in I_{b a c} \cup I_{b c a} \cup I_{c b a}$ would yield outcome $a$. Hence, for $i \in I_{b a c} \cup I_{b c a} \cup I_{c b a},(0,1,1 / 2)$ is the unique best response and thus undominated in any game $\Gamma\left(\succ_{I}, V^{\prime}\right)$ where $\hat{v} \in V^{\prime} \subseteq V^{1}$.

Hence, for $i \in I \backslash I_{a b c}$, no strategy used in $v, \hat{v}$ is dominated in any game $\Gamma\left(\succ_{I}, V^{\prime}\right)$ where $v, \hat{v} \in V^{\prime} \subseteq V^{1}$. It remains to check that for $i \in I_{a b c},(1,0,1 / 2)$ and $(1,1 / 2,0)$ are likewise undominated.

For that, consider profile $v$ where some $i \in I_{a b c}$ chooses $v_{i}=(1,0,1 / 2)$ and assume that $c \triangleright b, a$, so that $c$ is elected. A switch by $i$ to $(1 / 2,1,0)$ would also yield $c$, as we would still have $v^{a}=v^{c}$ and $v^{a}>v^{b}$. On the other hand, a switch to $(1,1 / 2,0)$ would yield $a$. Hence, for $i \in I_{a b c},(1,1 / 2,0)$ is the unique best response and thus undominated in any game $\Gamma\left(\succ_{I}, V^{\prime}\right)$ where $v, \hat{v} \in V^{\prime} \subseteq V^{1}$. 
Similarly, consider profile $\hat{v}$ where some $i \in I_{a b c}$ chooses $\hat{v}_{i}=(1,1 / 2,0)$ and assume that $b \triangleright a, c$, so that $b$ is elected. A switch to $(1 / 2,1,0)$ would still yield $b$, as we would now have $\hat{v}^{b}>\hat{v}^{a}$ and $\hat{v}^{a}>\hat{v}^{c}$. On the other hand, a switch to $(1,0,1 / 2)$ would yield $a$. Hence, for $i \in I_{a b c},(1,0,1 / 2)$ is the unique best response and thus undominated in any game $\Gamma\left(\succ_{I}, V^{\prime}\right)$ where $v, \hat{v} \in V^{\prime} \subseteq V^{1}$.

Case 2: $\left|I_{a b c}\right|=\left|I_{a c b}\right|+\left|I_{b a c}\right|+\left|I_{b c a}\right|+\left|I_{c a b}\right|+\left|I_{c b a}\right|+n$ with $0<n<\left|I_{b c a}\right|+$ $\left|I_{c a b}\right|+\left|I_{c b a}\right|$.

We will show that iterative elimination of dominated strategies cannot narrow down the set of strategies beyond $V_{a b c}^{\prime \prime}=V_{a b c}^{1}, V_{a c b}^{\prime \prime}=\{(1 / 2,0,1)\}, V_{b a c}^{\prime \prime}=\{(0,1,1 / 2)\}$, $V_{b c a}^{\prime \prime}, V_{c a b}^{\prime \prime}, V_{c b a}^{\prime \prime}=\{(0,1 / 2,1)\}$ and that $V^{\prime \prime}$ allows for the election of any candidate.

Let us construct $v \in V^{\prime \prime}$ as follows:

- $\left|I_{a c b}\right|+n$ of $I_{a b c}$ choose $v_{i}=(1 / 2,1,0)$

- $\left|I_{b a c}\right|+n$ of $I_{a b c}$ choose $v_{i}=(1,0,1 / 2)$

- $\left|I_{b c a}\right|+\left|I_{c a b}\right|+\left|I_{c b a}\right|-n$ of $I_{a b c}$ choose $v_{i}=(1,1 / 2,0)$

- All $i \in I_{a c b}$ choose $v_{i}=(1 / 2,0,1)$

- All $i \in I_{b a c}$ choose $v_{i}=(0,1,1 / 2)$

- All $i \in I_{b c a} \cup I_{c a b} \cup I_{c b a}$ choose $v_{i}=(0,1 / 2,1)$

Then, $v^{a}=v^{b}=v^{c}$ and any candidates may win. By Claim $1(1,0,1 / 2),(1,1 / 2,0) \in$ $V_{a b c}^{\prime \prime},(0,1,1 / 2) \in V_{b a c}^{\prime \prime}$ and $(0,1 / 2,1) \in V_{c a b}^{\prime \prime}, V_{c b a}^{\prime \prime}$ are undominated in any game $\Gamma\left(\succ_{I}, V^{\prime}\right)$ where $V^{\prime \prime} \subseteq V^{\prime} \subseteq V^{1}$.

To see that $(1 / 2,1,0) \in V_{a b c}^{\prime \prime}$ is undominated, let some $j \in I_{a b c}$ who chooses $v_{j}=(1,1 / 2,0)$ switch to $\tilde{v}_{j}=(1 / 2,1,0)$. Then $\tilde{v}^{a}=1 / 2|I|-1 / 2, \tilde{v}^{b}=1 / 2|I|+1 / 2$, and $\tilde{v}^{c}=1 / 2|I|$, so that by Claim $2(1 / 2,1,0) \in V_{a b c}^{\prime \prime}$ is undominated in any game $\Gamma\left(\succ_{I}, V^{\prime}\right)$ where $V^{\prime \prime} \subseteq V^{\prime} \subseteq V^{1}$.

To see that $(1 / 2,0,1) \in V_{a c b}^{\prime \prime}$ is undominated, note that $\left|I_{a c b}\right|>0$ implies $n \leq$ $\left|I_{b c a}\right|+\left|I_{c a b}\right|+\left|I_{c b a}\right|-2$ as condition (3) is violated by assumption. Then there exist $j, l \in I_{a b c}$ who play $v_{j, l}=(1,1 / 2,0)$. Let them switch to $\tilde{v}_{j}=(1,0,1 / 2)$ and $\tilde{v}_{l}=(1 / 2,1,0)$. Then $\tilde{v}^{a}=1 / 2|I|-1 / 2, \tilde{v}^{b}=1 / 2|I|$, and $\tilde{v}^{c}=1 / 2|I|+1 / 2$, so that by Claim $2(1 / 2,0,1) \in V_{a c b}^{\prime \prime}$ is undominated in any game $\Gamma\left(\succ_{I}, V^{\prime}\right)$ where $V^{\prime \prime} \subseteq V^{\prime} \subseteq V^{1}$

Finally, to see that $(0,1 / 2,1) \in V_{b c a}^{\prime \prime}$ is undominated, let some $j \in I_{a b c}$ switch from $v_{j}=(1 / 2,1,0)$ to $\tilde{v}_{j}=(1,0,1 / 2)$. Then $\tilde{v}^{a}=\tilde{v}^{c}=1 / 2|I|+1 / 2$ and $\tilde{v}^{b}=1 / 2|I|-1$, so that $c$ may win. Any deviation by $i$ to $(1 / 2,1,0),(0,1,1 / 2) \in V_{b c a}^{1}$ would elect candidate $a$ instead. Hence $(0,1 / 2,1) \in V_{b c a}^{\prime \prime}$ is undominated in any game $\Gamma\left(\succ_{I}, V^{\prime}\right)$ where $V^{\prime \prime} \subseteq V^{\prime} \subseteq V^{1}$.

For the remainder, we write $\left|I_{a}\right|:=\left|I_{a b c}\right|+\left|I_{a c b}\right|$ and define $\left|I_{b}\right|$ and $\left|I_{c}\right|$ accordingly.

Case 3: $\left|I_{a b c}\right|=\left|I_{a c b}\right|+\left|I_{b a c}\right|+\left|I_{b c a}\right|+\left|I_{c a b}\right|+\left|I_{c b a}\right|-n$ with $n \geq 0$ and $\left|I_{a}\right|>\left|I_{b}\right|+\left|I_{c}\right|$.

Then $\left|I_{a c b}\right|>\frac{n}{2} \geq 0$ and, as condition (3) is violated by assumption,

$$
\begin{aligned}
\left|I_{a c b}\right| \leq\left|I_{a b c}\right|= & \left|I_{a c b}\right|+\left|I_{b a c}\right|+\left|I_{b c a}\right|+\left|I_{c a b}\right|+\left|I_{c b a}\right| \\
& -n \leq\left|I_{a c b}\right|+\left|I_{b a c}\right|+2\left|I_{b c a}\right|+2\left|I_{c a b}\right|+2\left|I_{c b a}\right|-2
\end{aligned}
$$


which implies $\left|I_{b}\right|+\left|I_{c}\right| \geq n$ and $\left|I_{b c a}\right|+\left|I_{c}\right|+n \geq 2$. We will consider five exhaustive sub-cases: (i) $n$ is even, (ii) $n$ is odd and $\left|I_{b c a}\right|+\left|I_{c}\right| \geq 2$, (iii) $n \geq 3$ is odd and $\left|I_{b a c}\right| \geq 2$, (iv) $n=1,\left|I_{b c a}\right|+\left|I_{c}\right|=1$ and $\left|I_{b a c}\right| \geq 1$, (v) $n=1,\left|I_{b c a}\right|+\left|I_{c}\right|=1$ and $\left|I_{b a c}\right|=0$.

Case 3.1: Let $n$ be even.

We will show that iterative elimination of dominated strategies cannot narrow down the set of strategies beyond $V_{a b c}^{\prime \prime}=V_{a b c}^{1}, V_{a c b}^{\prime \prime}=\{(1 / 2,0,1),(1,0,1 / 2)\}, V_{b a c}^{\prime \prime}=$ $\{(0,1,1 / 2)\}, V_{b c a}^{\prime \prime}=\{(0,1 / 2,1),(0,1,1 / 2)\}$ and $V_{c a b}^{\prime \prime}, V_{c b a}^{\prime \prime}=\{(0,1 / 2,1)\}$, and that $V^{\prime \prime}$ allows for the election of any candidate.

Let us construct $v \in V^{\prime \prime}$ as follows:

- $\left|I_{a c b}\right|-\frac{n}{2}=1 / 2\left(\left|I_{a}\right|-\left|I_{b}\right|-\left|I_{c}\right|\right)$ of $I_{a b c}$ choose $v_{i}=(1 / 2,1,0)$

- $\left|I_{a c b}\right|-\frac{n}{2}=1 / 2\left(\left|I_{a}\right|-\left|I_{b}\right|-\left|I_{c}\right|\right)$ of $I_{a c b}$ choose $v_{i}=(1 / 2,0,1)$

- $\left|I_{b a c}\right|$ of $I_{a}$ choose $v_{i}=(1,0,1 / 2)$

- $\left|I_{b a c}\right|$ of $I_{b a c}$ choose $v_{i}=(0,1,1 / 2)$

- $\left|I_{b c a}\right|+\left|I_{c}\right|$ of $I_{a}$ choose $v_{i}=(1,1 / 2,0)$

- $\left|I_{b c a}\right|+\left|I_{c}\right|$ of $I_{b c a} \cup I_{c a b} \cup I_{c b a}$ choose $v_{i}=(0,1 / 2,1)$

Then, $v^{a}=v^{b}=v^{c}$ so that any candidate may win the election. By Claim 1, $(0,1,1 / 2) \in V_{b a c}^{\prime \prime}$ and $(0,1 / 2,1) \in V_{c a b}^{\prime \prime}, V_{c b a}^{\prime \prime}$ are undominated in any game $\Gamma\left(\succ_{I}, V^{\prime}\right)$ where $V^{\prime \prime} \subseteq V^{\prime} \subseteq V^{1}$-trivially if $\left|I_{b a c}\right|,\left|I_{c}\right|=0$.

To see that $(1,1 / 2,0) \in V_{a b c}^{\prime \prime}$ is likewise undominated, note that this follows from Claim 1 if $\left|I_{b c a}\right|+\left|I_{c}\right|>0$ (so that one can let some $i \in I_{a b c}$ choose $(1,1 / 2,0)$ at $v$ ). If instead $\left|I_{b c a}\right|+\left|I_{c}\right|=0$ then, as $n \geq 2$, there is some $i \in I_{a b c}$ who chooses $v_{i}=(1 / 2,1,0)$ which may elect $c$-only a switch to $\tilde{v}_{i}=(1,1 / 2,0)$ rules out $c$.

To see that $(1,0,1 / 2) \in V_{a b c}^{\prime \prime}$ is undominated, note that this follows from Claim 1 if $\left|I_{b a c}\right|>0$ (so that one can let some $i \in I_{a b c}$ chooses $(1,0,1 / 2)$ ) at $v$ ). If instead $\left|I_{b a c}\right|=0$ then, as $\left|I_{b}\right| \geq\left|I_{c}\right|$, we know that $\left|I_{b c a}\right| \geq 1$. Consider $v$ constructed such that some $i \in I_{a b c}$ chooses $v_{i}=(1,1 / 2,0)$ and let her switch to $\tilde{v}_{i}=(1,0,1 / 2)$ while some $j \in I_{b c a}$ switches from $v_{j}=(0,1 / 2,1)$ to $\tilde{v}_{j}=(0,1,1 / 2)$. Then $\tilde{v}^{a}=\tilde{v}^{b}=\tilde{v}^{c}$ and by Claim $1,(1,0,1 / 2) \in V_{a b c}^{\prime \prime}$ is undominated in any game $\Gamma\left(\succ_{I}, V^{\prime}\right)$ where $V^{\prime \prime} \subseteq V^{\prime} \subseteq V^{1}$

To see that $(1 / 2,1,0) \in V_{a b c}^{\prime \prime}$ is undominated, first consider $\left|I_{b c a}\right|+\left|I_{c}\right|>0$ and $v$ constructed such that $j \in I_{a b c}$ chooses $v_{j}=(1,1 / 2,0)$. Letting $j$ switch to $\tilde{v}_{j}=(1 / 2,1,0)$ gives $\tilde{v}^{a}=1 / 2|I|-1 / 2, \tilde{v}^{b}=1 / 2|I|+1 / 2$ and $\tilde{v}^{c}=1 / 2|I|$ so that by Claim 2, $(1 / 2,1,0) \in V_{a b c}^{\prime \prime}$ is undominated in any game $\Gamma\left(\succ_{I}, V^{\prime}\right)$ where $V^{\prime \prime} \subseteq V^{\prime} \subseteq V^{1}$. If instead $\left|I_{b c a}\right|+\left|I_{c}\right|=0$, then $n,\left|I_{b a c}\right| \geq 2$, so that $v$ may be constructed such that some $j \in I_{a b c}$ and $k \in I_{a c b}$ choose $v_{j}, v_{k}=(1,0,1 / 2)$. Letting them switch to $\tilde{v}_{j}=(1,1 / 2,0)$ and $\tilde{v}_{k}=(1 / 2,0,1)$ gives $\tilde{v}^{a}=1 / 2|I|-1 / 2$, $\tilde{v}^{b}=1 / 2|I|+1 / 2$ and $\tilde{v}^{c}=1 / 2|I|$ so that by Claim $2,(1 / 2,1,0) \in V_{a b c}^{\prime \prime}$ is undominated as before.

To see that $(1,0,1 / 2) \in V_{a c b}^{\prime \prime}$ is undominated, consider $i \in I_{a c b}$ who chooses $v_{i}=(1 / 2,0,1)$ which may elect $b$. Only a switch to $\tilde{v}_{i}=(1,0,1 / 2)$ rules it out.

To see that $(1 / 2,0,1) \in V_{a c b}^{\prime \prime}$ is undominated, first consider $\left|I_{b c a}\right|+\left|I_{c}\right| \geq 2$ and $v$ constructed such that $j \in I_{a b c}$ and $k \in I_{a b c} \cup I_{a c b}$ choose $v_{j}, v_{k}=(1,1 / 2,0)$. Letting them switch to $\tilde{v}_{j}=(1 / 2,1,0)$ and $\tilde{v}_{k}=(1,0,1 / 2)$ gives $\tilde{v}^{a}=1 / 2|I|-1 / 2$, 
$\tilde{v}^{b}=1 / 2|I|$ and $\tilde{v}^{c}=1 / 2|I|+1 / 2$ so that by Claim $2(1 / 2,0,1) \in V_{a b c}^{\prime \prime}$ is undominated in any game $\Gamma\left(\succ_{I}, V^{\prime}\right)$ where $V^{\prime \prime} \subseteq V^{\prime} \subseteq V^{1}$. If instead $\left|I_{b c a}\right|+\left|I_{c}\right| \leq 1$, then $n \geq 1$, so $n \geq 2$, so $\left|I_{b a c}\right|>0$. Hence, we may construct $v$ such that some $i \in I_{a c b}$ chooses $v_{i}=(1,0,1 / 2)$. Letting her switch to $\tilde{v}_{i}=(1 / 2,0,1)$ gives $\tilde{v}^{a}=1 / 2|I|-1 / 2$, $\tilde{v}^{b}=1 / 2|I|$ and $\tilde{v}^{c}=1 / 2|I|+1 / 2$ so that by Claim $2(1 / 2,0,1) \in V_{a b c}^{\prime \prime}$ is undominated as before.

To see that $(0,1 / 2,1),(0,1,1 / 2) \in V_{b c a}^{\prime \prime}$ are undominated, note that this is vacuously true if $\left|I_{b c a}\right|=0$. Assume instead that $\left|I_{b c a}\right| \geq 1$ and construct $v$ such that some $j \in$ $I_{a b c}$ chooses $v_{j}=(1,1 / 2,0)$. Letting $j$ switch to $\tilde{v}_{j}=(1,0,1 / 2)$ gives $\tilde{v}^{a}=1 / 2|I|$, $\tilde{v}^{b}=1 / 2|I|-1 / 2$ and $\tilde{v}^{c}=1 / 2|I|+1 / 2$ so that by Claim $2,(0,1 / 2,1) \in V_{b c a}^{\prime \prime}$ is undominated in any game $\Gamma\left(\succ_{I}, V^{\prime}\right)$ where $V^{\prime \prime} \subseteq V^{\prime} \subseteq V^{1}$. Moreover, letting in addition $i \in I_{b c a}$ switch to $\hat{v}_{i}=(0,1,1 / 2)$ gives $\left|\hat{v}^{a}\right|=\left|\hat{v}^{b}\right|=\left|\hat{v}^{c}\right|$ so that by Claim $1,(0,1,1 / 2) \in V_{b c a}^{\prime \prime}$ is likewise undominated.

Case 3.2: Let $n$ be odd, $\left|I_{b c a}\right|+\left|I_{c}\right| \geq 2$.

We will show that iterative elimination of dominated strategies cannot narrow down the set of strategies beyond $V_{a b c}^{\prime \prime}=V_{a b c}^{1}, V_{a c b}^{\prime \prime}=V_{a c b}^{1}, V_{b a c}^{\prime \prime}=\{(0,1,1 / 2)\}$ and $V_{b c a}^{\prime \prime}, V_{c a b}^{\prime \prime}, V_{c b a}^{\prime \prime}=\{(0,1 / 2,1)\}$, and that $V^{\prime \prime}$ allows for the election of any candidate.

First, let us construct $v \in V^{\prime \prime}$ as follows:

- $\left|I_{a c b}\right|-\frac{n}{2}+1 / 2=1 / 2\left(\left|I_{a}\right|-\left|I_{b}\right|-\left|I_{c}\right|\right)+1 / 2$ of $I_{a b c}$ choose $v_{i}=(1 / 2,1,0)$

- $\left|I_{a c b}\right|-\frac{n}{2}-1 / 2=1 / 2\left(\left|I_{a}\right|-\left|I_{b}\right|-\left|I_{c}\right|\right)-1 / 2$ of $I_{a c b}$ choose $v_{i}=(1 / 2,0,1)$

- $\left|I_{b a c}\right|+1$ of $I_{a}$ choose $v_{i}=(1,0,1 / 2)$

- $\left|I_{b a c}\right|$ of $I_{b a c}$ choose $v_{i}=(0,1,1 / 2)$

- $\left|I_{b c a}\right|+\left|I_{c}\right|-1$ of $I_{a}$ choose $v_{i}=(1,1 / 2,0)$

- $\left|I_{b c a}\right|+\left|I_{c}\right|$ of $I_{b c a} \cup I_{c a b} \cup I_{c b a}$ choose $v_{i}=(0,1 / 2,1)$

Then $v^{a}=v^{b}=v^{c}$ and any candidate may win. By Claim $1,(0,1,1 / 2) \in V_{b a c}^{\prime \prime}$ and $(0,1,1 / 2) \in V_{c a b}^{\prime \prime}, V_{c b a}^{\prime \prime}$ are undominated in any game $\Gamma\left(\succ_{I}, V^{\prime}\right)$ where $V^{\prime \prime} \subseteq$ $V^{\prime} \subseteq V^{1}$ - the latter holds trivially if $\left|I_{c}\right|=0$. Moreover, by assigning $i \in I_{a b c}$ or $j \in I_{a c b}$ to choose $(1,1 / 2,0)$ or $(1,0,1 / 2)$ in the construction of $v$, Claim 1 implies that $(1,1 / 2,0),(1,0,1 / 2) \in V_{a b c}^{\prime \prime}, V_{a c b}^{\prime \prime}$ are undominated.

Note that $\left|I_{a b c}\right|>\left|I_{a c b}\right|-\frac{n}{2}+1 / 2$ as $n \geq 1$ and $\left|I_{a b c}\right| \geq\left|I_{a c b}\right|$-if we had $n=1$ and $\left|I_{a b c}\right|=\left|I_{a c b}\right|$, then $\left|I_{b}\right|+\left|I_{c}\right|=1$, contradicting $\left|I_{b c a}\right|+\left|I_{c}\right| \geq 2$. Hence, we may construct $v$ with some $i \in I_{a b c}$ choosing a strategy other than $(1 / 2,1,0)$.

To see that $(1 / 2,1,0) \in V_{a b c}^{\prime \prime}$ is undominated, construct $v$ such that some $i \in I_{a b c} \subseteq$ $I_{a}$ chooses $v_{i}=(1,1 / 2,0)$. Letting $i$ switch to $\tilde{v}_{i}=(1 / 2,1,0)$ gives $\tilde{v}^{a}=1 / 2|I|-1 / 2$, $\tilde{v}^{b}=1 / 2|I|+1 / 2$ and $\tilde{v}^{c}=1 / 2|I|$ so that by Claim $2,(1 / 2,1,0) \in V_{a b c}^{\prime \prime}$ is undominated in any game $\Gamma\left(\succ_{I}, V^{\prime}\right)$ where $V^{\prime \prime} \subseteq V^{\prime} \subseteq V^{1}$.

To see that $(1 / 2,0,1) \in V_{a c b}^{\prime \prime}$ is undominated, construct $v$ such that some $i \in I_{a c b}$ chooses $v_{i}=(1,0,1 / 2)$. Letting $i$ switch to $\tilde{v}_{i}=(1 / 2,0,1)$ gives $\tilde{v}^{a}=1 / 2|I|-1 / 2$, $\tilde{v}^{b}=1 / 2|I|$ and $\tilde{v}^{c}=1 / 2|I|+1 / 2$ so that by Claim $2,(1 / 2,0,1) \in V_{a c b}^{\prime \prime}$ is undominated in any game $\Gamma\left(\succ_{I}, V^{\prime}\right)$ where $V^{\prime \prime} \subseteq V^{\prime} \subseteq V^{1}$.

To see that $(0,1 / 2,1) \in V_{b c a}^{\prime \prime}$ is undominated, construct $v$ such that some $j \in I_{a b c}$ chooses $v_{j}=(1,1 / 2,0)$. Letting $j$ switch to $\tilde{v}_{j}=(1,0,1 / 2)$ gives $\tilde{v}^{a}=1 / 2|I|$, $\tilde{v}^{b}=1 / 2|I|-1 / 2$ and $\tilde{v}^{c}=1 / 2|I|+1 / 2$ so that by Claim $2,(0,1 / 2,1) \in V_{b c a}^{\prime \prime}$ is undominated in any game $\Gamma\left(\succ_{I}, V^{\prime}\right)$ where $V^{\prime \prime} \subseteq V^{\prime} \subseteq V^{1}$. 
Case 3.3: Let $n \geq 3$ be odd and $\left|I_{b a c}\right| \geq 2$.

We will show that iterative elimination of dominated strategies cannot narrow down the set of strategies beyond $V_{a b c}^{\prime \prime}=V_{a b c}^{1}, V_{a c b}^{\prime \prime}=V_{a c b}^{1}, V_{b a c}^{\prime \prime}=\{(0,1,1 / 2)\}$ and $V_{b c a}^{\prime \prime}, V_{c a b}^{\prime \prime}, V_{c b a}^{\prime \prime}=\{(0,1 / 2,1)\}$, and that $V^{\prime \prime}$ allows for the election of any candidate.

First, let us construct $v \in V^{\prime \prime}$ as follows:

- $\left|I_{a c b}\right|-\frac{n}{2}-1 / 2=1 / 2\left(\left|I_{a}\right|-\left|I_{b}\right|-\left|I_{c}\right|\right)-1 / 2$ of $I_{a b c}$ choose $v_{i}=(1 / 2,1,0)$

- $\left|I_{a c b}\right|-\frac{n}{2}+1 / 2=1 / 2\left(\left|I_{a}\right|-\left|I_{b}\right|-\left|I_{c}\right|\right)+1 / 2$ of $I_{a c b}$ choose $v_{i}=(1 / 2,0,1)$

- $\left|I_{b a c}\right|-1$ of $I_{a}$ choose $v_{i}=(1,0,1 / 2)$

- $\left|I_{b a c}\right|$ of $I_{b a c}$ choose $v_{i}=(0,1,1 / 2)$

- $\left|I_{b c a}\right|+\left|I_{c}\right|+1$ of $I_{a}$ choose $v_{i}=(1,1 / 2,0)$

- $\left|I_{b c a}\right|+\left|I_{c}\right|$ of $I_{b c a} \cup I_{c a b} \cup I_{c b a}$ choose $v_{i}=(0,1 / 2,1)$

Then $v^{a}=v^{b}=v^{c}$ and any candidate may win. By Claim 1, we know that $(0,1,1 / 2) \in V_{b a c}^{\prime \prime}$ and $(0,1 / 2,1) \in V_{c a b}^{\prime \prime}, V_{c b a}^{\prime \prime}$ are undominated in any game $\Gamma\left(\succ_{I}, V^{\prime}\right)$ where $V^{\prime \prime} \subseteq V^{\prime} \subseteq V^{1}$-the latter holds trivially if $\left|I_{c}\right|=0$. Moreover, by assigning $i \in I_{a b c}$ or $j \in I_{a c b}$ to choose $(1,1 / 2,0)$ or $(1,0,1 / 2)$ in the construction of $v$, Claim 1 implies that $(1,1 / 2,0),(1,0,1 / 2) \in V_{a b c}^{\prime \prime}, V_{a c b}^{\prime \prime}$ are undominated.

To see that $(1 / 2,1,0) \in V_{a b c}^{\prime \prime}$ is undominated, construct $v$ such that some $i \in I_{a b c} \subseteq$ $I_{a}$ chooses $v_{i}=(1,1 / 2,0)$. Letting $i$ switch to $\tilde{v}_{j}=(1 / 2,1,0)$ gives $\tilde{v}^{a}=1 / 2|I|-1 / 2$, $\tilde{v}^{b}=1 / 2|I|+1 / 2$ and $\tilde{v}^{c}=1 / 2|I|$ so that by Claim $2,(1 / 2,1,0) \in V_{a b c}^{\prime \prime}$ is undominated in any game $\Gamma\left(\succ_{I}, V^{\prime}\right)$ where $V^{\prime \prime} \subseteq V^{\prime} \subseteq V^{1}$.

To see that $(1 / 2,0,1) \in V_{a c b}^{\prime \prime}$ is undominated, construct $v$ such that some $i \in I_{a c b} \subseteq$ $I_{a}$ chooses $v_{i}=(1,0,1 / 2)$. Letting $i$ switch to $\tilde{v}_{i}=(1 / 2,0,1)$ gives $\tilde{v}^{a}=1 / 2|I|-1 / 2$, $\tilde{v}^{b}=1 / 2|I|$ and $\tilde{v}^{c}=1 / 2|I|+1 / 2$ so that by Claim $2,(1 / 2,0,1) \in V_{a c b}^{\prime \prime}$ is undominated in any game $\Gamma\left(\succ_{I}, V^{\prime}\right)$ where $V^{\prime \prime} \subseteq V^{\prime} \subseteq V^{1}$.

To see that $(0,1 / 2,1) \in V_{b c a}^{\prime \prime}$ is undominated (in case $\left.\left|I_{b c a}\right|>0\right)$, construct $v$ such that some $j \in I_{a b c}$ chooses $v_{j}=(1,1 / 2,0)$. Letting $j$ switch to $\tilde{v}_{j}=(1,0,1 / 2)$ gives $\tilde{v}^{a}=1 / 2|I|, \tilde{v}^{b}=1 / 2|I|-1 / 2$ and $\tilde{v}^{c}=1 / 2|I|+1 / 2$ so that by Claim $2,(0,1 / 2,1) \in V_{b c a}^{\prime \prime}$ is undominated in any game $\Gamma\left(\succ_{I}, V^{\prime}\right)$ where $v \in V^{\prime} \subseteq V^{1}$.

Case 3.4: Let $n=1,\left|I_{b c a}\right|+\left|I_{c}\right|=1$ and $\left|I_{b a c}\right| \geq 1$.

We will show that iterative elimination of dominated strategies cannot narrow down the set of strategies beyond $V_{a b c}^{\prime \prime}=V_{a b c}^{1}, V_{a c b}^{\prime \prime}=\{(1 / 2,0,1)\}, V_{b a c}^{\prime \prime}=$ $\{(1 / 2,1,0),(0,1,1 / 2)\}$ and $V_{b c a}^{\prime \prime}, V_{c a b}^{\prime \prime}, V_{c b a}^{\prime \prime}=\{(0,1 / 2,1)\}$, and that $V^{\prime \prime}$ allows for the election of any candidate.

First, let us construct $v \in V^{\prime \prime}$ as follows:

- $\left|I_{a c b}\right|$ of $I_{a b c}$ choose $v_{i}=(1 / 2,1,0)$

- $\left|I_{a c b}\right|$ of $I_{a c b}$ choose $v_{i}=(1 / 2,0,1)$

- $\left|I_{b a c}\right|$ of $I_{a b c}$ choose $v_{i}=(1,0,1 / 2)$

- one of of $I_{b a c}$ choose $v_{i}=(1 / 2,1,0)$

- remainder of $I_{b a c}$ chooses $v_{i}=(0,1,1 / 2)$

- $\left|I_{b c a}\right|+\left|I_{c}\right|=1$ of $I_{b c a} \cup I_{c a b} \cup I_{c b a}$ choose $v_{i}=(0,1 / 2,1)$

Then $v^{a}=v^{b}=v^{c}$ and any candidate may win. By Claim $1,(1,0,1 / 2) \in V_{a b c}^{\prime \prime}$, $(1 / 2,1,0) \in V_{b a c}^{\prime \prime}$ and $(0,1,1 / 2) \in V_{c a b}^{\prime \prime}, V_{c b a}^{\prime \prime}$ are undominated in any game $\Gamma\left(\succ_{I}, V^{\prime}\right)$ where $V^{\prime \prime} \subseteq V^{\prime} \subseteq V^{1}$. 
To see that $(1 / 2,1,0) \in V_{a b c}^{\prime \prime}$ is undominated, let $j \in I_{a b c}$ switch from $v_{j}=$ $(1,0,1 / 2)$ to $\tilde{v}_{j}=(1,1 / 2,0)$ and $k \in I_{b a c}$ switch from $v_{k}=(1 / 2,1,0)$ to $\tilde{v}_{k}=$ $(0,1,1 / 2)$. Then $\tilde{v}^{a}=1 / 2|I|-1 / 2, \tilde{v}^{b}=1 / 2|I|+1 / 2$ and $\tilde{v}^{c}=1 / 2|I|$ so that by Claim $2,(1 / 2,1,0) \in V_{a b c}^{\prime \prime}$ is undominated in any game $\Gamma\left(\succ_{I}, V^{\prime}\right)$ where $V^{\prime \prime} \subseteq V^{\prime} \subseteq V^{1}$.

To see that $(1,1 / 2,0) \in V_{a b c}^{\prime \prime}$ is undominated, consider $i \in I_{a b c}$ who chooses $v_{i}=(1 / 2,1,0)$. A switch to $\tilde{v}_{i}=(1,1 / 2,0)$ elects $a$ for sure (rather than having it tied with $b$ and $c$ ), while a switch to $(1,0,1 / 2)$ may elect $c$, as $a$ and $c$ would be tied.

To see that $(1 / 2,0,1) \in V_{a c b}^{\prime \prime}$ is undominated, let $j \in I_{b a c}$ switch from $v_{j}=$ $(1 / 2,1,0)$ to $\tilde{v}_{j}=(0,1,1 / 2)$. Then $\tilde{v}^{a}=1 / 2|I|-1 / 2, \tilde{v}^{b}=1 / 2|I|$ and $\tilde{v}^{c}=1 / 2|I|+1 / 2$ so that by Claim $2,(1 / 2,0,1) \in V_{a c b}^{\prime \prime}$ is undominated in any game $\Gamma\left(\succ_{I}, V^{\prime}\right)$ where $V^{\prime \prime} \subseteq V^{\prime} \subseteq V^{1}$.

To see that $(0,1,1 / 2) \in V_{b a c}^{\prime \prime}$ is undominated, let some $i \in I_{b a c}$ switch from $v_{i}=(1 / 2,1,0)$ to $\tilde{v}_{i}=(0,1,1 / 2), j \in I_{a b c}$ from $v_{j}=(1,0,1 / 2)$ to $\tilde{v}_{j}=(1,1 / 2,0)$ and $k \in I_{a b c}$ from $v_{k}=(1 / 2,1,0)$ to $\tilde{v}_{k}=(1,1 / 2,0)$. Then $\tilde{v}^{a}=\tilde{v}^{b}=\tilde{v}^{c}=1 / 2|I|$ so that by Claim $1,(0,1,1 / 2) \in V_{b a c}^{\prime \prime}$ is undominated in any game $\Gamma\left(\succ_{I}, V^{\prime}\right)$ where $V^{\prime \prime} \subseteq V^{\prime} \subseteq V^{1}$

To see that $(0,1 / 2,1) \in I_{b c a}$ is undominated, let some $j \in I_{a b c}$ switch from $v_{j}=(1 / 2,1,0)$ to $\tilde{v}_{j}=(1,1 / 2,0)$ and some $k \in I_{b a c}$ from $v_{k}=(1 / 2,1,0)$ to $\tilde{v}_{k}=(0,1,1 / 2)$. Then $\tilde{v}^{a}=1 / 2|I|, \tilde{v}^{b}=1 / 2|I|-1 / 2$ and $\tilde{v}^{c}=1 / 2|I|+1 / 2$ so that by Claim 2, $(0,1 / 2,1) \in I_{b c a}$ is undominated in any game $\Gamma\left(\succ_{I}, V^{\prime}\right)$ where $V^{\prime \prime} \subseteq V^{\prime} \subseteq V^{1}$

Case 3.5: Let $n=1,\left|I_{b c a}\right|+\left|I_{c}\right|=1$ and $\left|I_{b a c}\right|=0$.

First not that our standing assumption $\left|I_{b}\right| \geq\left|I_{c}\right|$ implies $\left|I_{b c a}\right|=1$ and $\left|I_{c}\right|=0$. Moreover, $\left|I_{a b c}\right|=\left|I_{a c b}\right|+\left|I_{b a c}\right|+\left|I_{b c a}\right|+\left|I_{c a b}\right|+\left|I_{c b a}\right|-n=\left|I_{a c b}\right|$. We will show that iterative elimination of dominated strategies cannot narrow down the set of strategies beyond $V_{a b c}^{\prime \prime}=\{(1,1 / 2,0),(1 / 2,1,0)\}, V_{a c b}^{\prime \prime}=\{(1,0,1 / 2),(1 / 2,0,1)\}$ and $V_{b c a}^{\prime \prime}=\{(0,1,1 / 2),(0,1 / 2,1)\}$, and that $V^{\prime \prime}$ allows for the election of any candidate.

First, let us construct $v \in V^{\prime \prime}$ as follows:

- $\left|I_{a b c}\right|-1$ of $I_{a b c}$ choose $v_{i}=(1 / 2,1,0)$

- $\left|I_{a c b}\right|$ of $I_{a c b}$ choose $v_{i}=(1 / 2,0,1)$

- one of $I_{a b c}$ choose $v_{i}=(1,1 / 2,0)$

- one of $I_{b c a}$ choose $v_{i}=(0,1,1 / 2)$

Then $v^{a}=v^{b}=v^{c}$ and any candidate may win. By Claim 1, $(1,1 / 2,0) \in V_{a b c}^{\prime \prime}$ and $(0,1,1 / 2) \in V_{b c a}^{\prime \prime}$ are undominated in any game $\Gamma\left(\succ_{I}, V^{\prime}\right)$ where $V^{\prime \prime} \subseteq V^{\prime} \subseteq V^{1}$.

Next, let $j \in I_{a b c}$ switch from $v_{j}=(1,1 / 2,0)$ to $\tilde{v}_{j}=(1 / 2,1,0)$. Then $\tilde{v}^{a}=$ $1 / 2|I|-1 / 2, \tilde{v}^{b}=1 / 2|I|+1 / 2$ and $\tilde{v}^{c}=1 / 2|I|$ and by Claim $2,(1 / 2,1,0) \in V_{a b c}^{\prime \prime}$ is undominated in any game $\Gamma\left(\succ_{I}, V^{\prime}\right)$ where $V^{\prime \prime} \subseteq V^{\prime} \subseteq V^{1}$.

To see that $(1 / 2,0,1) \in V_{a c b}^{\prime \prime}$ is undominated, consider $v$ and let $i \in I_{a b c}$ switch from $v_{i}=(1,1 / 2,0)$ to $\tilde{v}_{i}=(1 / 2,1,0), j \in I_{b c a}$ from $v_{j}=(0,1,1 / 2)$ to $\tilde{v}_{j}=$ $(0,1 / 2,1)$. Then $\tilde{v}^{a}=1 / 2|I|-1 / 2, \tilde{v}^{b}=1 / 2|I|$ and $\tilde{v}^{c}=1 / 2|I|+1 / 2$ and by Claim 2 , $(1 / 2,0,1) \in V_{a c b}^{\prime \prime}$ is undominated in any game $\Gamma\left(\succ_{I}, V^{\prime}\right)$ where $V^{\prime \prime} \subseteq V^{\prime} \subseteq V^{1}$.

To see that $(1,0,1 / 2) \in V_{a c b}^{\prime \prime}$ is undominated, consider $v$ and let $i \in I_{a c b}$ switch from $v_{i}=(1 / 2,0,1)$ to $\tilde{v}_{i}=(1,0,1 / 2), j \in I_{a b c}$ from $v_{j}=(1,1 / 2,0)$ to $\tilde{v}_{j}=(1 / 2,1,0)$ and 
$k \in I_{b c a}$ from $v_{k}=(0,1,1 / 2)$ to $\tilde{v}_{k}=(0,1 / 2,1)$. Then $\tilde{v}^{a}=\tilde{v}^{b}=\tilde{v}^{c}$ and by Claim $1,(1,0,1 / 2) \in V_{a c b}^{\prime \prime}$ is undominated in any game $\Gamma\left(\succ_{I}, V^{\prime}\right)$ where $V^{\prime \prime} \subseteq V^{\prime} \subseteq V^{1}$.

To see that $(0,1 / 2,1) \in V_{b c a}^{\prime \prime}$ is undominated, consider $v$ and let $i \in I_{b c a}$ switch from $v_{i}=(0,1,1 / 2)$ to $\tilde{v}_{i}=(0,1 / 2,1)$. Then $\tilde{v}^{a}=1 / 2|I|, \tilde{v}^{b}=1 / 2|I|-1 / 2$ and $\tilde{v}^{c}=1 / 2|I|+1 / 2$ and by Claim $2,(0,1 / 2,1) \in V_{b c a}^{\prime \prime}$ is undominated in any game $\Gamma\left(\succ_{I}, V^{\prime}\right)$ where $V^{\prime \prime} \subseteq V^{\prime} \subseteq V^{1}$.

Case 4: $\left|I_{a b c}\right|=\left|I_{a c b}\right|+\left|I_{b a c}\right|+\left|I_{b c a}\right|+\left|I_{c a b}\right|+\left|I_{c b a}\right|-n$ with $n \geq 0$ and $\left|I_{a}\right| \leq\left|I_{b}\right|+\left|I_{c}\right|$.

First, consider $\left|I_{c}\right|=0$ so that $\left|I_{a}\right|=\left|I_{b}\right|$. If all $i \in I_{a}$ choose $v_{i}=(1,0,1 / 2)$ and all $i \in I_{b}$ choose $v_{i}=(0,1,1 / 2)$ then $v^{a}=v^{b}=v^{c}$, any candidate may win, and, by Claim 1, all strategies used in $v$ are undominated in any game $\Gamma\left(\succ_{I}, V^{\prime}\right)$ where $v \in V^{\prime} \subseteq V^{1}$.

Finally, consider $\left|I_{c}\right| \geq 1$ and construct $v$ as follows.

- $\left|I_{b}\right|+\left|I_{c}\right|-\left|I_{a}\right|$ of $I_{a}$ choose $v_{i}=(1,1 / 2,0)$

- $\left|I_{b}\right|+\left|I_{c}\right|-\left|I_{a}\right|$ of $I_{b}$ choose $v_{i}=(0,1,1 / 2)$

- $\left|I_{b}\right|+\left|I_{c}\right|-\left|I_{a}\right|$ of $I_{c}$ choose $v_{i}=(1 / 2,0,1)$

- $\left|I_{a}\right|-\left|I_{c}\right|$ of $I_{a}$ choose $v_{i}=(1,0,1 / 2)$

- $\left|I_{a}\right|-\left|I_{c}\right|$ of $I_{b}$ choose $v_{i}=(0,1,1 / 2)$

- $\left|I_{a}\right|-\left|I_{b}\right|$ of $I_{a}$ choose $v_{i}=(1,1 / 2,0)$

- $\left|I_{a}\right|-\left|I_{b}\right|$ of $I_{c}$ choose $v_{i}=(0,1 / 2,1)$

Then, $v^{a}=v^{b}=v^{c}$ and any candidate may win. Further, by Claim 1, all strategies used at $v$ are undominated in any game $\Gamma\left(\succ_{I}, V^{\prime}\right)$ where $v \in V^{\prime} \subseteq V^{1}$, hence survive iterative elimination of dominated strategies. This completes the Proof of Theorem 1.

Proof of Theorem 2 We first consider positional scoring rules with $s<\frac{1}{2}$ and show that for any fixed $s$, there exist preference profiles with $I=I_{a b c} \cup I_{a c b}$, where $b$ or $c$ may still be elected after iterated elimination of dominated strategies.

Let $\left|I_{a b c}\right|=\left|I_{a c b}\right|=n$ with $n>\frac{1-s}{1-2 s}$. We will show that the strategies $v_{i}=(s, 1,0) \in V_{a b c}$ and $v_{j}=(s, 0,1) \in V_{a c b}$ survive the iterative elimination of dominated strategies.

Consider $\Gamma\left(\succ_{I}, V^{1}\right)$ and a strategy profile $v$ where all $i \in I_{a b c}$ chose $v_{i}=(s, 1,0)$ while all $j \in I_{a c b}$ chose $v_{j}=(s, 0,1)$. Then $v^{b}=v^{c}=n$ and $v^{c}-v^{a}=n(1-2 s)>$ $1-s>0$ and either $b$ or $c$ is elected, depending on $\triangleright$. If some $i \in I_{a b c}$ would switch to a different strategy, $\tilde{v}_{i}=(1, s, 0),(1,0, s) \in V_{a b c}^{1}, c$ would win as it now has a higher score than $b$ and the gap $v^{c}-v^{a}$ has been narrowed by at most $1-s$.

Hence, $v_{i}=(s, 1,0)$ is undominated and included in $V_{a b c}^{2}$. A symmetric argument applies to $j \in I_{a c b}$ for whom $v_{j}=(s, 0,1) \in V_{a c b}^{2}$. By induction, $v_{i}$ and $v_{j}$, and hence outcomes $b$ and $c$, are never eliminated. This concludes the proof for the case $s<\frac{1}{2}$.

Next, we consider Antiplurality, i.e., $s=1$. Assume all voters agree on the ranking $a \succ_{i} b \succ_{i} c$, so that $V_{i}^{1}=\{(1,1,0),(1,0,1)\}$. If in $\Gamma\left(\succ_{I}, V^{1}\right)$ all voters $j \neq i$ chose $v_{j}=(1,1,0)$, then $i$ can ensure the election of $a$ by casting the ballot $v_{i}=(1,0,1)$, whereas $v_{i}^{\prime}=(1,1,0)$ would elect $b$ for $b \triangleright a$. In the same way, if all $j \neq i$ cast ballot 
$v_{j}=(1,0,1)$ and $c \triangleright a, i$ 's unique best reply is $v_{i}=(1,1,0)$. Hence, neither strategy is dominated and $a$ may fail to be elected.

Last, consider the case $s \in\left(\frac{1}{2}, 1\right)$. Assume $I=I_{a b c} \cup I_{b a c}$ and $\left|I_{a b c}\right|=n+1>$ $n=\left|I_{b a c}\right|$ with $n$ sufficiently large, so that in particular

$$
n>\frac{1-s}{2 s-1} \quad \text { and } \quad n>\frac{1}{2(1-s)} .
$$

We will show that the strategies $(1, s, 0),(1,0, s) \in V_{a b c}^{1}$ and $(s, 1,0),(0,1, s) \in V_{b a c}^{1}$ survive under iterative elimination so that $c$ remains a possible outcome: if all $i \in I_{a b c}$ vote $v_{i}=(1,0, s)$ while all $j \in I_{b a c}$ vote $v_{j}=(0,1, s)$, candidates scores are

$$
v^{a}=n+1, \quad v^{b}=n, \quad v^{c}=(2 n+1) s
$$

and $(\star \star)$ ensures that $v^{c}>v^{a}, v^{b}$.

Towards a proof by induction, recall that the sets of undominated strategies are

$$
V_{a b c}^{1}=\{(1, s, 0),(1,0, s),(s, 1,0)\} \quad \text { and } \quad V_{b a c}^{1}=\{(s, 1,0),(0,1, s),(1, s, 0)\}
$$

For the inductive step, we show that for arbitrary $m \geq 1$,

$$
\{(1, s, 0),(1,0, s)\} \subseteq V_{a b c}^{m} \quad \text { and } \quad\{(s, 1,0),(0,1, s)\} \subseteq V_{b a c}^{m}
$$

implies $\{(1, s, 0),(1,0, s)\} \subseteq V_{a b c}^{m+1}$ and $\{(s, 1,0),(0,1, s)\} \subseteq V_{b a c}^{m+1}$.

Part 1: $(1, s, 0) \in V_{a b c}^{m+1}$

Consider the situation where all $i \in I_{b a c}$ choose $v_{i}=(0,1, s)$. If all $j \in I_{a b c}$ choose $v_{j}=(1,0, s), c$ is elected since $(2 n+1) s>n+1$. If instead all $j \in I_{a b c}$ choose $v_{j}^{\prime}=(1, s, 0), b$ wins. Hence, $(1, s, 0)$ is not dominated by $(1,0, s)$.

Next, assume that $(s, 1,0) \in V_{a b c}^{m}$ and consider the situation where all $i \in I_{b a c}$ choose $v_{i}=(s, 1,0)$. If all $j \in I_{a b c}$ choose $v_{j}=(s, 1,0), b$ is elected. If instead all $j \in I_{a b c}$ choose $v_{j}^{\prime}=(1, s, 0), a$ wins. Hence, $(1, s, 0)$ is not dominated by $(s, 1,0)$. We conclude that $(1, s, 0) \in V_{a b c}^{m+1}$.

Part 2: $(1,0, s) \in V_{a b c}^{m+1}$

Consider the situation where $n-1$ voters $i \in I_{b a c}$ choose $v_{i}=(s, 1,0)$ and one $j \in I_{b a c}$ chooses $v_{j}=(0,1, s)$. If all $k \in I_{a b c}$ choose $v_{k}=(1,0, s), a$ is elected, as $v^{a}>n(1+s)>v^{b}, v^{c}$. If instead all $k \in I_{a b c}$ choose $v_{k}^{\prime}=(1, s, 0)$ or $v_{k}^{\prime}=(s, 1,0)$, $b$ is elected. Hence, $v_{k}=(1,0, s) \in V_{a b c}^{m+1}$.

Part 3: $(0,1, s) \in V_{b a c}^{m+1}$

Consider the situation where all $i \in I_{a b c}$ choose $v_{i}=(1, s, 0)$. If all $j \in I_{b a c}$ choose $v_{j}=(0,1, s), b$ is elected, as $v^{b}>n(1+s)>v^{a}, v^{c}$. If instead all $j \in I_{b a c}$ choose $v_{j}^{\prime}=(s, 1,0)$ or $v_{j}^{\prime}=(1, s, 0), a$ is elected. Hence, $v_{j}=(0,1, s) \in V_{b a c}^{m+1}$.

Part 4: $(s, 1,0) \in V_{b a c}^{m+1}$ 
$i \in I_{a b c}$ choose $v_{i}=(1,0, s)$. If all $j \in I_{b a c}$ choose $v_{j}=(0,1, s), c$ is elected. If instead all $j \in I_{b a c}$ choose $v_{j}^{\prime}=(s, 1,0), a$ wins. Hence, $v_{j}=(s, 1,0)$ is not dominated by $(0,1, s)$. If $(1, s, 0) \notin V_{b a c}^{m}$, this completes the proof.

If $(1, s, 0) \in V_{b a c}^{m}$, consider the situation where all $i \in I_{a b c}$ choose $v_{i}=(1, s, 0)$ and one $j \in I_{b a c}$ chooses $(0,1, s)$. If all $n-1$ remaining voters $k \in I_{b a c}$ choose $v_{k}=(1, s, 0), a$ is elected as by $(\star \star), v^{a}=2 n>2 n s+1=v^{b}$. If instead the remaining $k \in I_{b a c}$ choose $v_{k}^{\prime}=(s, 1,0), b$ is elected as by $s>\frac{1}{2}, v^{b}=n+n s+s>$ $n+n s+(1-s)=v^{a}$. Hence, $v_{k}=(s, 1,0)$ is not dominated by $(1, s, 0)$. We conclude that $(s, 1,0) \in V_{b a c}^{m+1}$.

Proof of Fact 2 Suppose (1) is satisfied. Then in $\Gamma\left(\succ_{I}, V^{1}\right)$, where each voter approves of their most but not of their least preferred candidate, $z$ receives a lower score than either $x$ or $y$ and is thus ruled out as an outcome. This makes it a dominated (or redundant) strategy in $\Gamma\left(\succ_{I}, V^{1}\right)$ to also approve of the less preferred candidate among the two remaining, $x$ and $y$. Removing these strategies, we find that the majoritypreferred among the two is the unique outcome. If instead the electorate is split, with equally many voters preferring $x$ over $y$ as $y$ over $x$, no further restriction of outcomes beyond $\{x, y\}$ can be achieved: voters in $I_{x y z} \cup I_{z x y}$ and $I_{y x z} \cup I_{z y x}$ are already left with a single strategy while for voters $i \in I_{x z y} \cup I_{y z x}$, their two remaining strategies (they may or may not approve of $z$ ) are redundant.

Suppose instead that (1) is not satisfied under any labelling of candidates. Assuming that $x$ is the candidate who is most preferred for most voters, i.e., $\left|I_{x y z}\right|+\left|I_{x z y}\right| \geq$ $\left|I_{y x z}\right|+\left|I_{y z x}\right|$ and $\left|I_{x y z}\right|+\left|I_{x z y}\right| \geq\left|I_{z x y}\right|+\left|I_{z y x}\right|$, construct a profile $v$ as follows: (i) only voters for whom $x$ is the most preferred approve of $x$, (ii) voters for whom $y$ is the most preferred candidate approve of it together with some voters for whom $y$ is the second most preferred, namely together with $\left|I_{x y z}\right|+\left|I_{x z y}\right|-\left|I_{y x z}\right|-\left|I_{y z x}\right|$ voters from $I_{x y z} \cup I_{z y x}$-if there were not enough voters $I_{x y z} \cup I_{z y x}$ to make this possible, then (1) would have been satisfied. In the same way, let (iii) all voters $i \in I_{z x y}+I_{z y x}$ as well as $\left|I_{x y z}\right|+\left|I_{x z y}\right|-\left|I_{z x y}\right|+\left|I_{z y x}\right|$ voters from $I_{x z y} \cup I_{y z x}$ approve of $z$. Then we have a three-way tie with $v^{x}=v^{y}=v^{z}=\frac{\left|I_{x y z}\right|+\left|I_{x z y}\right|}{2}$. For a voter who approves of only her most-preferred candidate at that profile a deviation rules out its election, so is a worse reply for some $\triangleright$. For a voter who also approves of her second-most-preferred candidate, a deviation rules out its election while preserving her least-preferred candidate as a possible outcome. Again, this is a worse reply for some $\triangleright$ - it may change the outcome from $b$ to $c$. Hence, the strategies used are (iteratively) undominated and no outcome can be ruled out.

Proof of Theorem 3 Let us first analyse scoring rules where $V$ consist of all permutations of $(1,1,0)$ and $(s, s, 0)$. For that, consider a preference profile where all voters share the same preferences, $I=I_{a b c}$.

Claim 3 For all $i$, if $V_{i}^{m}$ includes at least one of the two ballots $(1,0,1)$ or $(s, 0, s)$ as well at least one of the two ballots $(1,1,0)$ or $(s, s, 0)$ then after eliminating dominated strategies in the game $\Gamma\left(\succ_{I}, V^{m}\right), V_{i}^{m+1}$ will contain at least one of the two ballots $(1,0,1)$ or $(s, 0, s)$ as well at least one of the two ballots $(1,1,0)$ or $(s, s, 0)$.

Proof. In the game $\Gamma\left(\succ_{I}, V^{m}\right)$, let $v$ be a ballot profile where all voters choose $(1,0,1)$ or $(s, 0, s)$ so that $v^{a}=v^{c}>v^{b}$ and $c$ is elected if $c \triangleright a$. If some voter $i$ 
switches to $\tilde{v}_{i}=(1,1,0)$ or $\tilde{v}_{i}=(s, s, 0)$, the outcome is $a$. If instead she switches to $(0,1,1)$ or $(0, s, s)$, provided these are still included in $V_{i}^{m}$, the outcome is $c$. Hence, at least one of the ballots $(1,1,0),(s, s, 0)$ is undominated and included in $V_{i}^{m+1}$.

Analogously, let $v$ be a profile where all voters choose $(1,1,0)$ or $(s, s, 0)$ so that $v^{a}=v^{b}>v^{c}$ and $b$ is elected if $b \triangleright a$. If some voter $i$ switches to $\tilde{v}_{i}=(1,0,1)$ or $\tilde{v}_{i}=(s, 0, s)$, the outcome is $a$. If instead she switches to $(0,1,1)$ or $(0, s, s)$, the outcome is $b$. Hence, at least one of the ballots $(1,0,1),(s, 0, s)$ is undominated and included in $V_{i}^{m+1}$.

Claim $3 \diamond$

Since initially they are included in the set of admissible ballots, at least one of $(1,1,0)$ and $(s, s, 0)$ survives under iterative elimination of dominated strategies. But then $b$ cannot be excluded as an outcome, so that MEW (as well as $\mathbf{U}$ ) is violated.

Next, let us analyse scoring rules where $V$ consist of all permutations of $(1,0,0)$ and $(s, 0,0)$. For $s=1$, we know by Theorem 2 that the Plurality rule violates $\mathbf{U}$. For $s<1$, consider a preference profile where $I=I_{a b c} \cup I_{a c b}$ and $\left|I_{a b c}\right|=\left|I_{a c b}\right| \geq 2$.

Claim 4 If $(0,1,0) \in V_{a b c}^{m}$ and $(0,0,1) \in V_{a c b}^{m}$, then both strategies are undominated in the game $\Gamma\left(\succ_{I}, V^{m}\right)$ and hence $(0,1,0) \in V_{a b c}^{m+1},(0,0,1) \in V_{a c b}^{m+1}$.

Proof. In the game $\Gamma\left(\succ_{I}, V^{m}\right)$, let $v$ be the ballot profile where all voters $i \in I_{a b c}$ choose $(0,1,0)$, all $j \in I_{a c b}$ choose $(0,0,1)$. Then $v^{b}=v^{c} \geq 2>0=v^{a}$ and $b \triangleright c$ yields outcome $b$. If any $i \in I_{a b c}$ deviates, $c$ wins. Thus, $v_{i}=(0,1,0)$ is undominated and included in $V_{a b c}^{m+1}$. Analogously, $(0,0,1)$ is included in $V_{a c b}^{m+1} . \quad$ Claim $4 \diamond$

By induction, $(0,1,0) \in V_{a b c}$ and $(0,0,1) \in V_{a c b}$ survive under iterative elimination of dominated strategies. Then $\mathbf{U}$ is violated as $b$ and $c$ cannot be excluded.

Now, let us consider vote-splitting scoring rules, i.e., where $V$ consists of all permutations of $(s, s, 0)$ and $(1-s, 0,0)$. We want to show that such a rule violates $\mathbf{U}$ if $s<1 / 2$. For that, consider a preference profile where $I=I_{a b c} \cup I_{a c b}$ and $\left|I_{a b c}\right|=\left|I_{a c b}\right| \geq 2$.

Claim 5 If $(0,1-s, 0) \in V_{a b c}^{m}$ and $(0,0,1-s) \in V_{a c b}^{m}$, then both strategies are undominated in the game $\Gamma\left(\succ_{I}, V^{m}\right)$ and hence $(0,1-s, 0) \in V_{a b c}^{m+1},(0,0,1-s) \in$ $V_{a c b}^{m+1}$.

Proof. In the game $\Gamma\left(\succ_{I}, V^{m}\right)$, let $v$ be the ballot profile where all voters $i \in I_{a b c}$ choose $(0,1-s, 0)$, all $j \in I_{a c b}$ choose $(0,0,1-s)$. Then $v^{b}=v^{c}>v^{a}$ and $b \triangleright c$ yields outcome $b$. If any $i \in I_{a b c}$ deviates, $c$ wins. Thus, $v_{i}=(0,1-s, 0)$ is undominated and included in $V_{a b c}^{m+1}$. Analogously, $(0,0,1-s)$ is included in $V_{a c b}^{m+1}$.

Claim $5 \diamond$

By induction, $(0,1-s, 0) \in V_{a b c}$ and $(0,0,1-s) \in V_{a c b}$ survive under iterative elimination of dominated strategies. Then $\mathbf{U}$ is violated as $b$ and $c$ cannot be excluded.

Finally, we want to show that a vote-splitting scoring rule violates MEW if $s>1 / 2$. For $s=1$, we know from Theorem 2 that the Antiplurality Rule violates MEW. For $s \in(1 / 2,1)$, consider a preference profile where $I=I_{a b c} \cup I_{b a c},\left|I_{a b c}\right|=n+1$, $\left|I_{b a c}\right|=n$ and $n$ sufficiently large, $n>\frac{1}{(1-s)(2 s-1)}$. We will show that strategies $(s, s, 0),(s, 0, s),(1-s, 0,0) \in V_{a b c}^{m}$ and $(0, s, s),(0,1-s, 0) \in V_{b a c}^{m}$ are undominated in $\Gamma\left(\succ_{I}, V^{m}\right)$ and hence included in $V_{a b c}^{m+1}$ and $V_{b a c}^{m+1}$ respectively. 
(i) For $i \in I_{a b c},(s, s, 0)$ is undominated in $\Gamma\left(\succ_{I}, V^{m}\right)$.

Consider a profile $v$ where

- $i$ votes $v_{i}=(s, s, 0)$

- one $j \in I_{a b c}$ votes $v_{j}=(s, 0, s)$

- remaining $n-1$ of $I_{a b c}$ vote $v_{j}=(1-s, 0,0)$

- all $n$ of $I_{b a c}$ vote $v_{j}=(0, s, s)$

Then $v^{b}=v^{c}=s(n+1)$ and $v^{a}=2 s+(1-s)(n-1)$, so that

$$
v^{a}-v^{b}=-s n+3 s+n-1-s n-s=(1-n)(2 s-1)<0
$$

and $b$ is elected for $b \triangleright c$. A switch by $i$ to any other ballot $\tilde{v}_{i} \in V$ would never raise the score of $a$ and would either reduce the score of $b$ or increase the score of $c$, thereby changing the outcome to $c$. Hence $(s, s, 0)$ is undominated.

(ii) For $i \in I_{a b c},(s, 0, s)$ is undominated.

Consider a ballot profile $v$ where

- $i$ votes $v_{i}=(s, 0, s)$

- $n$ of $I_{a b c}$ vote $v_{j}=(1-s, 0,0)$

- $n-\left\lfloor\frac{s}{2 s-1}\right\rfloor$ of $I_{b a c}$ vote $v_{j}=(0,1-s, 0)^{33}$

- $\left\lfloor\frac{s}{2 s-1}\right\rfloor$ of $I_{b a c}$ vote $v_{j}=(0, s, s)$

Then

$$
v^{a}-v^{b}=s-(2 s-1)\left\lfloor\frac{s}{2 s-1}\right\rfloor \in[0,2 s-1) .
$$

Moreover, $v^{a}-v^{c}=n(1-s)-s\left\lfloor\frac{s}{2 s-1}\right\rfloor>\frac{1}{2 s-1}-\frac{s^{2}}{2 s-1}>0$ so that and $a$ is elected for $a \triangleright b$. A switch by $i$ to ballot $(1-s, 0,0)$ would change the score difference $v^{a}-v^{b}$ by $-s+(1-s)=1-2 s$ so that $b$ overtakes $a$. As any other ballot would change the difference $v^{a}-v^{b}$ even more in $b$ 's favour, we conclude that $(s, 0, s)$ is undominated.

(iii) For $i \in I_{a b c},(1-s, 0,0)$ is not dominated by $(s, 0, s)$ or $(0,0,1-s)$.

Let $v$ be the ballot profile where all $i \in I_{a b c}$ vote $v_{i}=(1-s, 0,0)$, all $j \in I_{b a c}$ vote $v_{j}=(0, s, s)$. Then $v^{b}=v^{c}=s n$ is larger than $v^{a}=(1-s)(1+n)$ as $s>1-s$ and $n$ is large. Then for $b \triangleright c, b$ wins while a switch by $i$ to $(s, 0, s)$ or $(0,0,1-s)$ yields $c$.

(iv) For $i \in I_{a b c},(1-s, 0,0)$ is not dominated by $(s, s, 0),(0, s, s)$ or $(0,1-s, 0)$.

Let $v$ be the ballot profile where all $j \in I_{a b c}$ vote $v_{j}=(1-s, 0,0)$, all $j \in I_{b a c}$ vote $v_{j}=(0,1-s, 0)$. Then $v^{a}-v^{b}=1-s, v^{c}=0$ and $a$ is elected. A switch by $i$ to $(s, s, 0)$ would yield $v^{a}=v^{b}$, so that for $b \triangleright a, a$ would no longer be elected. A switch by $i$ to $(0, s, s)$ or $(0,1-s, 0)$ would likewise yield outcome $b$.

(v) For $i \in I_{b a c},(0, s, s)$ is undominated.

Let $v$ be a ballot profile where one $j \in I_{a b c}$ votes $v_{j}=(s, s, 0), n$ of $I_{a b c}$ vote $v_{j}=(1-s, 0,0)$ and all $j \in I_{b a c}$ vote $v_{j}=(0,1-s, 0)$. Then $v^{a}=v^{b}$ and $v^{c}=0$

$33\lfloor x\rfloor$ denotes the largest integer weakly smaller than $x ;\lceil x\rceil$ the smallest weakly larger integer. 
so that for $a \triangleright b, a$ is elected. For $i \in I_{b a c}$, only a switch to $(0, s, s)$ would increase the difference $v^{b}-v^{a}$ and hence yield outcome $b$.

(vi) For $i \in I_{b a c},(0,1-s, 0)$ is not dominated by $(s, s, 0)$ (while $\left.(s, s, 0) \in V_{b a c}^{m}\right)$.

If $(s, s, 0) \in V_{b a c}^{m}$, consider the ballot profile $v$ where every voter chooses $(s, s, 0)$. Then if $a \triangleright b$, candidate $a$ is elected. A switch by $i$ to $(0,1-s, 0)$ yields outcome $b$.

(vii) For $i \in I_{b a c},(0,1-s, 0)$ is not dominated by $(1-s, 0,0),(0,0,1-s)$, $(0, s, s)$ or $(s, 0, s)$.

Consider a ballot profile $v$ where

- One $j \in I_{a b c}$ votes $(s, 0, s)$,

- $n$ of $I_{a b c}$ vote $(1-s, 0,0)$,

- $\left\lceil\frac{s}{1-s}\right\rceil>1$ of $I_{b a c}$ vote $(0,1-s, 0)$,

- $n-\left\lceil\frac{s}{1-s}\right\rceil$ of $I_{b a c}$ vote $(0, s, s)$.

Then

$$
v^{b}-v^{c}=(1-s)\left\lceil\frac{s}{1-s}\right\rceil-s \in[0,1-s)
$$

and

$$
v^{a}-v^{c}=n(1-2 s)+s\left\lceil\frac{s}{1-s}\right\rceil<-\frac{1}{1-s}+s\left(\frac{s}{1-s}+1\right)=\frac{s-1}{1-s}<0
$$

so the $b$ is elected for $b \triangleright c$. If $i \in I_{b a c}$ switches from $(0,1-s, 0)$ to either $(1-s, 0,0)$, $(0,0,1-s),(0, s, s)$ or $(s, 0, s)$, she reduces the payoff difference $v^{b}-v^{c}$ by at least $1-s$, so that $c$ 's score is now higher than $b$ 's, ruling out $b$ as an outcome.

Together, (i)-(vii) establish by induction that $(s, s, 0) \in V_{a b c}$ and $(0, s, s) \in V_{b a c}$ are iteratively undominated. Thus $b$ remains a possible outcome, violating MEW.

Proof of Theorem 5 We will now complete the proof for the only if part, i.e., show that any non-EAV-rule $V$ fails MEPD. Recall our definitions

$$
\underline{s}=\min \{s \mid(1, s, 0) \in V)\} \quad \text { and } \quad \bar{s}=\max \{s \mid(1, s, 0) \in V)\} .
$$

As $V$ is a non-EAV-rule, $\underline{s}>0$ or $\bar{s}<0$. Section 4.2 considered the case $\underline{s}+\bar{s}<1$ under the assumption $\underline{s}, \bar{s} \in \mathbb{Q}$. To deal with irrational $\underline{s}$ or $\bar{s}$, we will make use of the following facts.

Claim 6 Let $x, y \in \mathbb{R}^{+}$. Then for any $\varepsilon>0$, there exist infinitely many $p, q \in \mathbb{N}$ such that $0 \leq p x-q y<\varepsilon$. In particular, for any $\varepsilon$ and any $M$ there are infinitely many $p, q>M$ that satisfy the inequality. Similarly, there are (infinitely many) $p, q$ such that $-\varepsilon<p x-q y \leq 0$.

To see this, rewrite the inequality as $0 \leq p-q \frac{y}{x}<\frac{\varepsilon}{x}$. For $\frac{y}{x} \in \mathbb{Q}$ the claim follows immediately. If instead $\frac{y}{x}$ is irrational, it follows from the fact that for any $r \in \mathbb{R} \backslash \mathbb{Q}$ the set $S:=\{q r-\lfloor q r\rfloor \mid q \in \mathbb{N}\}$ is dense in [0,1]-one can then find (infinitely many) $q$ such that $q \frac{y}{x}-\left\lfloor q \frac{y}{x}\right\rfloor \in\left(1-\frac{\varepsilon}{x}, 1\right]$ and set $p=\left\lfloor q \frac{y}{x}\right\rfloor+1$. 
Claim 7 Let $x, y \in \mathbb{R}^{+}$and $\frac{x}{y} \notin \mathbb{Q}$. Then for any $\varepsilon>0$, there exist infinitely many $p, q \in \mathbb{N}$ such that $\varepsilon \leq p x-q y<2 \varepsilon$. In particular, for any $\varepsilon$ and any $M$ there are infinitely many $p, q>M$ that satisfy the inequality.

Rewrite the inequality as $\frac{\varepsilon}{x} \leq p-q \frac{y}{x}<\frac{2 \varepsilon}{x}$. Then it follows from the fact that for any $r \in \mathbb{R} \backslash \mathbb{Q}$ the set $S:=\{q r-\lfloor q r\rfloor \mid q \in \mathbb{N}\}$ is dense in $[0,1]$-one can then find (infinitely many) $q$ such that $q \frac{y}{x}-\left\lfloor q \frac{y}{x}\right\rfloor \in\left(1-\frac{2 \varepsilon}{x}, 1-\frac{\varepsilon}{x}\right\rfloor$ and set $p=\left\lfloor q \frac{y}{x}\right\rfloor+1$.

As a final preparatory observation, note that since $V$ is finite, there is a minimal distance in assigned scores, i.e., there exists $\delta>0$ such that for any two ballots and any candidate for whom they differ in their assigned score, they differ by at least $\delta$.

Case 1: $\underline{s}+\bar{s}<1$, with $\underline{s}$ or $\bar{s}$ in $\mathbb{R} \backslash \mathbb{Q}$. We will show that iterative elimination of dominated strategies cannot narrow down the set of strategies beyond $V_{a b c}^{\prime \prime}=$ $\{(\bar{s}, 1,0),(1,0, \underline{s}),(1, \underline{s}, 0)\}$ and $V_{b c a}^{\prime \prime}=\{(0, \bar{s}, 1),(0,1, \bar{s}),(\underline{s}, 1,0)\}$.

For that, consider $\left|I_{a b c}\right|=n_{1}+n_{2}+n_{3}$ and $\left|I_{b c a}\right|=m+n_{1}+n_{2}+n_{3}$ for some $m, n_{1}, n_{2}, n_{3} \in \mathbb{N}$ and $0<m \leq n_{2}+n_{3}$ to be determined later. By MEPD, $b$ should be the unique winner for $m>0$. Now, construct $v$ as follows.

- $m$ of $I_{a b c}$ choose $v_{i}=(1,0, \underline{s})$

- $n_{1}$ of $I_{a b c}$ choose $v_{i}=(\bar{s}, 1,0)$

- $n_{2}+n_{3}-m$ of $I_{a b c}$ choose $v_{i}=(1, \underline{s}, 0)$

- $m$ of $I_{b c a}$ choose $v_{i}=(\underline{s}, 1,0)$

- $n_{1}$ of $I_{b c a}$ choose $v_{i}=(0,1, \bar{s})$

- $n_{2}+n_{3}$ of $I_{b c a}$ choose $(0, \bar{s}, 1)$

Then $v^{a}=v^{c}=m \cdot \underline{s}+n_{1} \cdot \bar{s}+n_{2}+n_{3}, v^{b}=m(1-\underline{s})+2 n_{1}+\left(n_{2}+n_{3}\right)(\underline{s}+\bar{s})$ and hence

$$
v^{a, c}-v^{b}=-m \underbrace{(1-2 \underline{s})}_{>0}-n_{1} \underbrace{(2-\bar{s})}_{>0}+\left(n_{2}+n_{3}\right) \underbrace{(1-\underline{s}-\bar{s})}_{>0} .
$$

By Claim 6 we find $m, n_{1}, n_{2}, n_{3}>M$, for any given $M$, such that $-m(1-2 \underline{s})+$ $n_{2}(1-\underline{s}-\bar{s}) \in\left[0, \frac{\delta}{4}\right)$ and $-n_{1}(2-\bar{s})+n_{3}(1-\underline{s}-\bar{s}) \in\left[0, \frac{\delta}{4}\right)$. Then $v^{a, c}-v^{b} \in\left[0, \frac{\delta}{2}\right)$. Moreover, $n_{3}$ can be chosen large enough (together with $n_{1}$ ) so that $n_{2}+n_{3}-m>M$. Denote the set of tuples $\left(m, n_{1}, n_{2}, n_{3}\right) \in \mathbb{N}^{4}$ for whom these conditions are satisfied as $P^{M}$.

Now construct the following additional profiles $\tilde{v}, \hat{v}$ from $v$ with $\left(m, n_{1}, n_{2}, n_{3}\right) \in$ $P^{M}$ by letting some voters switch to different strategies. By choosing $M$ large enough we can ensure that each construction will be well defined, i.e., that the number of voters initially using a strategy at $v$ is larger than the number of voters who switch away from that strategy in the construction.

If $\frac{1-\underline{s}}{1-\bar{s}}$ is irrational, construct $\tilde{v}$ from $v$ by letting, $k$ of $I_{a b c}$ switch from $(1, \underline{s}, 0)$ to $(\bar{s}, 1,0)$ and $l$ of $I_{b c a}$ from $(0,1, \bar{s})$ to $(0, \bar{s}, 1)$. Then $\tilde{v}^{b}-\tilde{v}^{c}=v^{b}-v^{c}+k(1-$ $\underline{s})-l 2(1-\bar{s})$ and $\tilde{v}^{c}-\tilde{v}^{a}=(k+l)(1-\bar{s})$. By Claim 7 , we can find $k, l$ such that $k(1-\underline{s})-l 2(1-\bar{s}) \in\left[\frac{\delta}{2}, \delta\right)$ so that $\tilde{v}^{b}-\tilde{v}^{c} \in(0, \delta)$. Moreover, choosing $k, l$ large, we have $\tilde{v}^{c}-\tilde{v}^{a}>2$.

If instead $\frac{1-\underline{s}}{1-\overline{\bar{s}}} \in \mathbb{Q}$ but $\frac{1+\underline{s}}{1-\overline{\bar{s}}} \notin \mathbb{Q}$, construct $\tilde{v}$ from $v$ by letting, $k$ of $I_{a b c}$ switch from $(1,0, \underline{s})$ to $(\bar{s}, 1,0)$ and $l$ of $I_{b c a}$ from $(0,1, \bar{s})$ to $(0, \bar{s}, 1)$. Then $\tilde{v}^{b}-\tilde{v}^{c}=$ 
$v^{b}-v^{c}+k(1+\underline{s})-l 2(1-\bar{s})$ and $\tilde{v}^{c}-\tilde{v}^{a}=k(1-\underline{s}-\bar{s})+l(1-\bar{s})$. By Claim 7 , we can find $k, l$ such that $k(1+\underline{s})-l 2(1-\bar{s}) \in\left[\frac{\delta}{2}, \delta\right)$ so that $\tilde{v}^{b}-\tilde{v}^{c} \in(0, \delta)$. Moreover, choosing $k, l$ large, we have $\tilde{v}^{c}-\tilde{v}^{a}>2$.

If instead $\frac{1-\underline{s}}{1-\overline{\bar{s}}} \in \mathbb{Q}$ and $\frac{1+\underline{s}}{1-\overline{\bar{s}}} \in \mathbb{Q}$, then $\frac{1}{1-\bar{s}} \in \mathbb{Q}$, hence $\bar{s} \in \mathbb{Q}$ and hence also $\underline{s} \in \mathbb{Q}$ - a case we have already dealt with in Sect. 4.2.

Construct $\hat{v}$ from $v$ by letting $k^{\prime}$ voters of $I_{a b c}$ switch from $(\bar{s}, 1,0)$ to $(1, \underline{s}, 0)$ and $k^{\prime}$ of $I_{b c a}$ switch from $(0,1, \bar{s})$ to $(0, \bar{s}, 1)$. Then $a$ and $c$ are still tied, but $\hat{v}^{a, c}-\hat{v}^{b}=$ $v^{a, c}-v^{b}+k^{\prime}(3-\underline{s}-2 \bar{s})$, so that for $k^{\prime}$ large, $\hat{v}^{a, c}-\hat{v}^{b}>2$.

To see that $(1, \underline{s}, 0) \in V_{a b c}^{\prime \prime}$ is undominated in any game $\Gamma\left(\succ_{I}, V^{\prime}\right)$ with $v \in$ $V^{\prime} \subseteq V^{1}$, consider a voter who uses that strategy in $v$. A move to any other strategy would either decrease the score of $a$ by at least $\delta$ or increase the score of $b$ or $c$ by at least $\delta$. Either way, $a$ would no longer be the outcome for $a \triangleright b, c$. In the same way, $(1,0, \underline{s}) \in V_{a b c}^{\prime \prime}$ is seen to be undominated as a move away from it (at $v$ ) would rule out outcome $a$. Also, $(\bar{s}, 1,0) \in V_{a b c}^{\prime \prime}$ is not dominated by any strategy $(s, 1,0), s<\bar{s}$.

To see that $(\bar{s}, 1,0) \in V_{a b c}^{\prime \prime}$ is not dominated by any other strategy, consider a voter who uses that strategy in $\tilde{v}$. Here $b$ is the unique outcome, but a lower score of $b$ or a higher score for $c$ (by at least $\delta$ ) would make $c$ the unique outcome.

To see that $(0,1, \bar{s}) \in V_{b c a}^{\prime \prime}$ is likewise undominated, consider a voter who uses it at $\tilde{v}$. Here $b$ is the unique outcome. Any strategy that reduces the score difference between $b$ and $c$ makes $c$ the unique winner. Moreover, it cannot be dominated by a strategy that weakly increases the score difference - it would then either reduce the score of $c$ or increase the score of $a$, so that in both cases a voter using that strategy instead of $(0,1, \bar{s})$ would make $a$ the unique outcome at $\hat{v}$.

To see that $(\underline{s}, 1,0) \in V_{b c a}^{\prime \prime}$ is likewise undominated, consider a voter who uses it at $\tilde{v}$. Here $b$ is the unique outcome and any other strategy that decreases the score of $b$ or increases the score of $c$ would make $c$ the unique winner. Also, a strategy $(s, 1,0)$ that assigned a higher score to $a$ would make $a$ the unique outcome at $\hat{v}$.

To see that $(0, \bar{s}, 1) \in V_{b c a}^{\prime \prime}$ is likewise undominated, consider a voter who uses it at $\hat{v}$. Here $c$ may win but any other strategy that decreases the score of $c$ or increases the score of $a$ would make $a$ the unique winner. Also, a strategy $(0, s, 1)$ that assigned a lower score to $b$ (by at least $\delta$ ) would rule out $b$ at $\tilde{v}$.

Case 2: $\underline{s}+\bar{s}>1$. We will show that iterative elimination of dominated strategies cannot narrow down the set of strategies beyond $V_{a b c}^{\prime \prime}=\{(\bar{s}, 1,0),(1,0, \underline{s}),(1, \underline{s}, 0)\}$ and $V_{b c a}^{\prime \prime}=\{(0, \bar{s}, 1),(0,1, \bar{s}),(\underline{s}, 1,0)\}$.

For that, consider $\left|I_{a b c}\right|=m+n_{1}+n_{2}+n_{3}$ and $\left|I_{b c a}\right|=n_{1}+n_{2}+n_{3}$ for some $m, n_{1}, n_{2}, n_{3} \in \mathbb{N}$ and $0<m \leq n_{1}+n_{2}$ to be determined later. By MEPD, $a$ should be the unique winner for $m>0$. Now, construct $v$ as follows.

- $m$ of $I_{a b c}$ choose $v_{i}=(\bar{s}, 1,0)$

- $n_{1}+n_{2}$ of $I_{a b c}$ choose $v_{i}=(1,0, \underline{s})$

- $n_{3}$ of $I_{a b c}$ choose $v_{i}=(1, \underline{s}, 0)$

- $m$ of $I_{b c a}$ choose $v_{i}=(0, \bar{s}, 1)$

- $n_{1}+n_{2}-m$ of $I_{b c a}$ choose $v_{i}=(0,1, \bar{s})$

- $n_{3}$ of $I_{b c a}$ choose $v_{i}=(\underline{s}, 1,0)$ 
Then $v^{a}=v^{b}=m \cdot \bar{s}+n_{1}+n_{2}+n_{3}(1+\underline{s}), v^{c}=m(1-\bar{s})+\left(n_{1}+n_{2}\right)(\underline{s}+\bar{s})$ and hence

$$
v^{a, b}-v^{c}=m \underbrace{(2 \bar{s}-1)}_{>0}-\left(n_{1}+n_{2}\right) \underbrace{(\underline{s}+\bar{s}-1)}_{>0}+n_{3} \underbrace{(1+\underline{s})}_{>0} .
$$

By Claim 6, for any $M$, there are $m, n_{1}, n_{2}, n_{3}>M$ such that $m(2 \bar{s}-1)-n_{1}(\underline{s}+$ $\bar{s}-1) \in\left[0, \frac{\delta}{4}\right)$ and $-n_{2}(\underline{s}+\bar{s}-1)+n_{3}(1+\underline{s}) \in\left[0, \frac{\delta}{4}\right)$, so that $v^{a, b}-v^{c} \in\left[0, \frac{\delta}{2}\right)$. Moreover, $n_{2}$ may be chosen large enough (together with $n_{3}$ ) such that $n_{1}+n_{2}>m$. Denote the set of tuples $\left(m, n_{1}, n_{2}, n_{3}\right) \in \mathbb{N}^{4}$ such that these conditions are satisfied as $P^{M}$.

At any profile $v$, with $\left(m, n_{1}, n_{2}, n_{3}\right) \in P^{M}$, there is either a tie between $a$ and $b$ (with $c$ within $\frac{\delta}{2}$-distance) or a tie involving all three candidates. Letting some voters in $I_{b c a}$ switch from $(\underline{s}, 1,0)$ to $(0, \bar{s}, 1)$ changes the election outcome to $c$. It remains to show that the strategies used in the construction of $v$ are iteratively undominated.

To see that, $v_{i}=(1,0, \underline{s}) \in V_{a b c}^{\prime \prime}$ is undominated in any game $\Gamma\left(\succ_{I}, V^{\prime}\right)$ where $v \in V^{\prime} \subseteq V^{1}$, observe that any other strategy would either decrease the score of $a$ or increase the score of $b$ or $c$ by at least $\delta$. Hence, any deviation from $v_{i}=(1,0, \underline{s})$ would preclude the election of $a$ and hence be worse for $i \in I_{a b c}$ (for $a \triangleright b, c$ ). In the same way, $(1, \underline{s}, 0) \in V_{a b c}^{\prime \prime}$ and $(0,1, \bar{s}),(\underline{s}, 1,0) \in V_{b c a}^{\prime \prime}$ are seen to be undominated in any game $\Gamma\left(\succ_{I}, V^{\prime}\right)$ where $v \in V^{\prime} \subseteq V^{1}$ as any deviation by a voter would rule out the election of their most preferred candidate.

To see that $(\bar{s}, 1,0) \in V_{a b c}^{\prime \prime}$ is undominated, construct $\tilde{v}$ from $v$ by letting $k$ of $I_{a b c}$ switch from $(1,0, \underline{s})$ to $(\bar{s}, 1,0)$ and $l$ from $I_{b c a}$ switch from $(\underline{s}, 1,0)$ to $(0, \bar{s}, 1)$. Then

$$
\begin{gathered}
\tilde{v}^{b}-\tilde{v}^{a}=k(2-\bar{s})+l(\underline{s}+\bar{s}-1) \quad \text { and } \\
\tilde{v}^{b}-\tilde{v}^{c}=v^{b}-v^{c}+k(1+\underline{s})-l(2-\bar{s}) .
\end{gathered}
$$

By Claim 6, we can find $k$ and $l$ such that $k(1+\underline{s})-l(2-\bar{s}) \in\left[0, \frac{\delta}{2}\right)$ and, with $k$ and $l$ large, $k(2-\bar{s})+l(\underline{s}+\bar{s}-1)>2$.

For such a pair $k, l$, set $\tilde{M}=\max \{k, l\}$. Then for any profile $v$ with $\left(m, n_{1}, n_{2}, n_{3}\right) \in$ $P^{\tilde{M}}, \tilde{v}$ is well defined as $n_{1}+n_{2}>\tilde{M} \geq k$ and $n_{3}>\tilde{M} \geq l$. Moreover, at $\tilde{v}$,

$$
\tilde{v}^{b}-\tilde{v}^{b}>2 \quad \text { and } \quad \tilde{v}^{b}-\tilde{v}^{c} \in[0, \delta)
$$

so that $b$ is either the unique outcome or tied with $c$. If a voter $i \in I_{a b c}$, who at $\tilde{v}$ chooses $(\bar{s}, 1,0)$, would deviate to any other strategy in $V^{1}$, it would either increase the score of $c$ or reduce the score of $b$ by at least $\delta$ (strategies $(s, 1,0)$ with $s<\bar{s}$ are dominated and hence not in $V_{a b c}^{1}$ ). Such a deviation would hence make $c$ the unique winner-thus $(\bar{s}, 1,0) \in V_{a b c}^{\prime \prime}$ is undominated in any game $\Gamma\left(\succ_{I}, V^{\prime}\right)$ where $v \in V^{\prime} \subseteq V^{1}$.

To see that $(0, \bar{s}, 1) \in V_{b c a}^{\prime \prime}$ is undominated, consider first $\bar{s}=1$. Then $(0, \bar{s}, 1)=$ $(0,1, \bar{s}) \in V_{b c a}^{\prime \prime}$ for which we have already seen that it is undominated as long as a profile $v$ with $\left(m, n_{1}, n_{2}, n_{3}\right) \in P^{M}$ is included in $V^{\prime} \subseteq V^{1}$. 
Now, consider $\bar{s}<1$. If $\frac{1+s}{1-\overline{\bar{s}}} \notin \mathbb{Q}$ construct $\hat{v}$ from $v$ by letting $k^{\prime}$ of $I_{a b c}$ switch from $(\bar{s}, 1,0)$ to $(1, \underline{s}, 0)$ and $l^{\prime}$ of $I_{b c a}$ switch from $(\underline{s}, 1,0)$ to $(0, \bar{s}, 1)$. Then

$$
\begin{gathered}
\hat{v}^{c}-\hat{v}^{b}=v^{c}-v^{b}+k^{\prime}(1-\underline{s})+l^{\prime}(2-\bar{s}) \quad \text { and } \\
\hat{v}^{c}-\hat{v}^{a}=v^{c}-v^{a}-k^{\prime}(1-\bar{s})+l^{\prime}(1+\underline{s}) .
\end{gathered}
$$

By Claim 7 we can find $k^{\prime}$ and $l^{\prime}$ such that $-k^{\prime}(1-\bar{s})+l^{\prime}(1+\underline{s}) \in\left[\frac{\delta}{2}, \delta\right)$ and, with $k^{\prime}$ and $l^{\prime}$ large, $\hat{v}^{c}-\hat{v}^{b}>2$.

For such a pair $k^{\prime}, l^{\prime}$, set $\hat{M}=\max \left\{k^{\prime}, l^{\prime}, \tilde{M}\right\}$. Then for any profile $v$ with $\left(m, n_{1}, n_{2}, n_{3}\right) \in P^{\hat{M}} \subseteq P^{\tilde{M}}, \hat{v}$ is well defined as $m>\hat{M} \geq k^{\prime}$ and $n_{3}>\hat{M} \geq l^{\prime}$. Moreover, at $\hat{v}$,

$$
\hat{v}^{c}-\hat{v}^{b}>2 \quad \text { and } \quad \hat{v}^{c}-\hat{v}^{a} \in(0, \delta)
$$

so that $c$ is the unique outcome. If a voter $i \in I_{b c a}$, who at $\hat{v}$ chooses $(0, \bar{s}, 1)$, would deviate to any other strategy in $V^{1}$, it would either decrease the score of $c$ or increase the score of $a$ by at least $\delta$ (strategies $(0, s, 1)$ with $s<\bar{s}$ are undominated and hence not in $V_{b c a}^{1}$ ). Such a deviation would hence make $a$ the unique winner-which shows that $(0, \bar{s}, 1) \in V_{b c a}^{\prime \prime}$ is undominated in any game $\Gamma\left(\succ_{I}, V^{\prime}\right)$ where $v \in V^{\prime} \subseteq V^{1}$.

If instead $\frac{1+\underline{s}}{1-\bar{s}} \in \mathbb{Q}$ but $\frac{\underline{s}}{1-\bar{s}} \notin \mathbb{Q}$ construct $\hat{v}$ from $v$ by letting $k^{\prime}$ of $I_{a b c}$ switch from $(\bar{s}, 1,0)$ to $(1, \underline{s}, 0)$ and $l^{\prime}$ of $I_{a b c}$ switch from $(1, \underline{s}, 0)$ to $(1,0, \underline{s})$. Then

$$
\begin{gathered}
\hat{v}^{c}-\hat{v}^{b}=v^{c}-v^{b}+k^{\prime}(1-\underline{s})+l^{\prime} 2 \underline{s} \quad \text { and } \\
\hat{v}^{c}-\hat{v}^{a}=v^{c}-v^{a}-k^{\prime}(1-\bar{s})+l^{\prime} \underline{s}
\end{gathered}
$$

By Claim 7 we can again find $k^{\prime}$ and $l^{\prime}$ such that $-k^{\prime}(1-\bar{s})+l^{\prime} \underline{s} \in\left[\frac{\delta}{2}, \delta\right)$ and, with $k^{\prime}$ and $l^{\prime}$ large, $\hat{v}^{c}-\hat{v}^{b}>2$ and complete the proof as before.

Finally, if both $\frac{1+\underline{s}}{1-\overline{\bar{s}}}, \frac{\underline{s}}{1-\bar{s}} \in \mathbb{Q}$, then also $\frac{1}{1-\bar{s}} \in \mathbb{Q}$. But then $\bar{s} \in \mathbb{Q}$ and thus also $\underline{s} \in \mathbb{Q}$. Then, we can find $v \in P^{\tilde{M}}$ such that $v^{a}=v^{b}=v^{c}$. Construct $\hat{v}$ from $v$ by letting, as before, $k^{\prime}$ of $I_{a b c}$ switch from $(\bar{s}, 1,0)$ to $(1, \underline{s}, 0)$ and $l^{\prime}$ of $I_{b c a}$ switch from $(\underline{s}, 1,0)$ to $(0, \bar{s}, 1)$. Then

$$
\hat{v}^{c}-\hat{v}^{b}=k^{\prime}(1-\underline{s})+l^{\prime}(2-\bar{s}) \quad \text { and } \quad \hat{v}^{c}-\hat{v}^{a}=-k^{\prime}(1-\bar{s})+l^{\prime}(1+\underline{s}) .
$$

Moreover, $k^{\prime}$ and $l^{\prime}$ may be chosen such that $\hat{v}^{c}-\hat{v}^{a}=0$ and $\hat{v}^{c}-\hat{v}^{b}>2$ and we can complete the proof as before.

Case 3: $\underline{s}+\bar{s}=1$. Once more, we will show that iterative elimination of dominated strategies cannot narrow down the set of strategies beyond $V_{a b c}^{\prime \prime}=$ $\{(\bar{s}, 1,0),(1,0, \underline{s}),(1, \underline{s}, 0)\}$ and $V_{b c a}^{\prime \prime}=\{(0, \bar{s}, 1),(0,1, \bar{s}),(\underline{s}, 1,0)\}$.

Let $\left|I_{a b c}\right|=m+n_{1}+n_{2}$ and $\left|I_{b c a}\right|=n_{1}+n_{2}$. Depending on $\underline{s}$ and $\bar{s}$ we will choose $m$ even or odd, but small, i.e., $m \in\{1,2\} ; n_{1}$ and $n_{2}$ will be chosen sufficiently large so that each of the following three ballot profiles is well defined. First, at $v$,

- $m+1+2 p$ of $I_{a b c}$ choose $(\bar{s}, 1,0)$ 
- $n_{1}-1-p$ of $I_{a b c}$ choose $(1,0, \underline{s})$

- $n_{2}-p$ of $I_{a b c}$ choose $(1, \underline{s}, 0)$

- 1 of $I_{b c a}$ chooses $(\underline{s}, 1,0)$

- $n_{1}-1-q$ of $I_{b c a}$ choose $(0,1, \bar{s})$

- $n_{2}+q$ of $I_{b c a}$ choose $(0, \bar{s}, 1)$

Then $v^{b}-v^{c}=m+2+2 p-q 2(1-\bar{s})$ and $v^{c}-v^{a}=-m \underline{s}-1+p(-2 \bar{s}-$ $\underline{s}+2)+q(1-\bar{s})=-\bar{s}(m+1)+\underline{s}(p+q)$. By Claim 6, we can find infinitely many $(m+2+2 p), q$ such that $v^{b}-v^{c} \in[0, \delta)$. Moreover, choosing $m \in\{1,2\}$ and $p, q$ sufficiently large yields $v^{c}-v^{a}>2$.

Next, write $n$ for the sum $n_{1}+n_{2}$ and construct $\tilde{v}$ as follows:

- $n+m-u$ of $I_{a b c}$ choose $(1, \underline{s}, 0)$

- $u$ of $I_{a b c}$ choose $(1,0, \underline{s})$

- $n-w$ of $I_{b c a}$ choose $(0, \bar{s}, 1)$

- $w$ of $I_{b c a}$ choose $(\underline{s}, 1,0)$

Then $\tilde{v}^{a}-\tilde{v}^{c}=(m+w)-(u-w) \underline{s}$ and $\tilde{v}^{a}-\tilde{v}^{b}=m(1-\underline{s})+u \underline{s}$ and by Claim 6 we can find infinitely many $u, w$, for any $m$ given by the construction of $v$ above, such that $\tilde{v}^{a}-\tilde{v}^{c} \in[0, \delta)$. Moreover, choosing $u$ large enough yields $v^{a}-v^{b}>2$. Analogously, for any given $m$, we may choose $u, w$ such that $\tilde{v}^{c}-\tilde{v}^{a} \in[0, \delta)$.

Finally, at $\hat{v}$

- $m$ of $I_{a b c}$ choose $(\bar{s}, 1,0)$

- $m+1$ of $I_{a b c}$ choose $(1,0, \underline{s})$

- $n-m-1$ of $I_{a b c}$ choose $(1, \underline{s}, 0)$

- $m$ of $I_{b c a}$ choose $(0, \bar{s}, 1)$

- 1 of $I_{b c a}$ choose $(0,1, \bar{s})$

- $n-m-1$ of $I_{b c a}$ choose $(\underline{s}, 1,0)$

Then $\hat{v}^{a}=\hat{v}^{b}=m(\bar{s}-\underline{s})+n(1+\underline{s})-\underline{s}$ and $\hat{v}^{c}=m(1+\underline{s})+1$. Hence $\hat{v}^{a}=\hat{v}^{b}$ and, for $n$ large enough, $\hat{v}^{a, b}-\hat{v}^{c}>2$.

For all constructions to be well defined, set $m \in\{1,2\}$ as required in the derivation of a (near) tie at $v$ and $n$ large enough to simultaneously satisfy all implicit lower bounds in the construction of the different profiles.

We are now ready to sum up: the exact tie of $a$ and $b$ at $\hat{v}$ shows $(1,0, \underline{s}) \in V_{a b c}^{\prime \prime}$ to be undominated in any game $\Gamma\left(\succ_{I}, V^{\prime}\right)$ where $V^{\prime \prime} \subseteq V^{\prime} \subseteq V^{1}$ : at $\hat{v}, a$ is a possible outcome but a deviation by a voter who uses $(1,0, \underline{s})$ in $\hat{v}$ to another ballot in $V_{a b c}^{1}$ rules out $a$. Similarly, $(1, \underline{s}, 0) \in V_{a b c}^{\prime \prime}$ is not dominated by any strategy in $V_{a b c}^{1}$.

To see that $(\bar{s}, 1,0) \in \bar{V}_{a b c}^{\prime \prime}$ is undominated in any game $\Gamma\left(\succ_{I}, V^{\prime}\right)$ where $V^{\prime \prime} \subseteq$ $V^{\prime} \subseteq V^{1}$, consider the near tie of $b$ and $c$ at $v$-any deviation within $V_{a b c}^{1}$ would lower the score of $b$ or increase the score of $c$ by at least $\delta$ and would hence make $c$ the unique outcome. In the same way, $v$ demonstrates that $(\underline{s}, 1,0) \in V_{b c a}$ is undominated.

To see that $(0,1, \bar{s})$ is not dominated by any ballot that yields a lower score difference between $b$ and $a$ consider the exact tie of $a$ and $b$ at $\hat{v}$. To see that it is not dominated by any ballot that yields a lower score difference between $c$ and $a$, consider the near tie of $c$ and $a$ ( $c$ with a weakly higher score than $a$ ) at $\tilde{v}$. In the same way, $(0, \bar{s}, 1) \in V_{b c a}^{\prime \prime}$ is seen to not be dominated by any strategy in $V_{b c a}^{1}$. 


\section{References}

Baharad, E., Nitzan, S.: Approval voting reconsidered. Econ. Theor. 26(3), 619-628 (2005)

Baharad, E., Nitzan, S.: Scoring rules: An alternative parameterization. Econ. Theor. 30(1), 187-190 (2007)

Barberà, S., Dutta, B., Sen, A.: Strategy-proof social choice correspondences. J. Econom. Theory 101(2), 374-394 (2001)

Barbie, M., Puppe, C., Tasnádi, A.: Non-manipulable domains for the Borda count. Econ. Theor. 27(2), 411-430 (2006)

Brams, S.J., Fishburn, P.C.: Approval voting. Am. Polit. Sci. Rev. 72(03), 831-847 (1978)

Buenrostro, L., Dhillon, A., Vida, P.: Scoring rule voting games and dominance solvability. Soc. Choice Welfare 40(2), 329-352 (2013)

Campbell, D.E., Kelly, J.S.: Gains from manipulating social choice rules. Econ. Theor. 40(3), 349-371 (2009)

Ching, S., Zhou, L.: Multi-valued strategy-proof social choice rules. Soc. Choice Welfare 19(3), 569-580 (2002)

Condorcet, M.d.: Essai sur l'application de l'analyse à la probabilité des décisions rendues à la pluralité des voix. L'imprimerie royale, Paris (1785)

Courtin, S., Núñez, M.: Dominance solvable approval voting games. J. Public Econom. Theory 19(6), 1055-1076 (2017)

Dhillon, A., Lockwood, B.: When are plurality rule voting games dominance-solvable? Games Econom. Behavior 46(1), 55-75 (2004)

Dietrich, F.: Scoring rules for judgment aggregation. Soc. Choice Welfare 42(4), 873-911 (2014)

Farquharson, R.: Theory of Voting. Blackwell, Oxford (1969)

Gärdenfors, P.: Manipulation of social choice functions. J. Econom. Theory 13(2), 217-228 (1976)

Gärdenfors, P.: On definitions of manipulation of social choice functions. In: Laffont, J.J. (ed.) Aggregation and Revelation of Preferences, pp. 29-36. North-Holland, Amsterdam (1979)

Gibbard, A.: Manipulation of voting schemes: a general result. Econometrica 41(4), 587-602 (1973)

Goertz, J.M., Maniquet, F.: On the informational efficiency of simple scoring rules. J. Econom. Theory 146(4), 1464-1480 (2011)

Kelly, J.S.: Almost all social choice rules are highly manipulable, but a few aren't. Soc. Choice Welfare 10(2), 161-175 (1993)

Lepelley, D., Merlin, V.: Scoring run-off paradoxes for variable electorates. Econ. Theor. 17(1), 53-80 (2001)

Marx, L.M., Swinkels, J.M.: Order independence for iterated weak dominance. Games Econom. Behavior 18(2), 219-245 (1997)

May, K.O.: A set of independent necessary and sufficient conditions for simple majority decision. Econometrica 20(4), 680-684 (1952)

Moulin, H.: Dominance solvable voting schemes. Econometrica 47(6), 1337-1351 (1979)

Moulin, H.: Choosing from a tournament. Soc. Choice Welfare 3(4), 271-291 (1986)

Moulin, H.: Axioms of Cooperative Decision Making. Econometric Society Monographs. Cambridge University Press, Cambridge (1991)

Myerson, R.B.: Axiomatic derivation of scoring rules without the ordering assumption. Soc. Choice Welfare 12(1), 59-74 (1995)

Myerson, R.B.: Comparison of scoring rules in poisson voting games. J. Econom. Theory 1(103), 219-251 (2002)

Núñez, M., Laslier, J.F.: Preference intensity representation: strategic overstating in large elections. Soc. Choice Welfare 42(2), 313-340 (2014)

Sanver, M.R.: Strategy-proofness of the plurality rule over restricted domains. Econ. Theor. 39(3), 461-471 (2009)

Sanver, M.R., Zwicker, W.S.: Monotonicity properties and their adaptation to irresolute social choice rules. Soc. Choice Welfare 39(2-3), 371-398 (2012)

Satterthwaite, M.A.: Strategy-proofness and arrow's conditions: Existence and correspondence theorems for voting procedures and social welfare functions. J. Econom. Theory 10(2), 187-217 (1975)

Young, P.: Optimal voting rules. J. Econom. Perspect. 9(1), 51-64 (1995)

Publisher's Note Springer Nature remains neutral with regard to jurisdictional claims in published maps and institutional affiliations. 